MobileCDP: A MOBILE FRAMEWORK FOR THE CONSUMER DECISION PROCESS

\author{
A THESIS SUBMITTED TO \\ THE GRADUATE SCHOOL OF INFORMATICS \\ OF \\ THE MIDDLE EAST TECHNICAL UNIVERSITY
}

BY

SÜLEYMAN ÖZARSLAN

IN PARTIAL FULFILLMENT OF THE REQUIREMENTS FOR THE DEGREE OF DOCTOR OF PHILOSOPHY

IN

THE DEPARTMENT OF INFORMATION SYSTEMS

MARCH 2015 



\section{MObileCDP: A MOBILE FRAMEWORK FOR THE CONSUMER DECISION PROCESS}

Submitted by SÜLEYMAN ÖZARSLAN in partial fulfillment of the requirements for the degree of Philosophy of Doctorate in Information Systems, Middle East Technical University by,

Prof. Dr. Nazife Baykal

Director, Informatics Institute

Prof. Dr. Yasemin Yardımcı Çetin

Head of Department, Information Systems

Assist. Prof. Dr. P. Erhan Eren

Supervisor, Information Systems, METU

Examining Committee Members:

Prof. Dr. Nazife Baykal

Information Systems, METU

Assist. Prof. Dr. P. Erhan Eren

Information Systems, METU

Assist. Prof. Dr. Murat Perit Çakır

Cognitive Science, METU

Assoc. Prof. Dr. Altan Koçyiğit

Information Systems, METU

Assoc. Prof. Dr. İbrahim Körpeoğlu

Computer Engineering, Bilkent University

Date:

02.03.2015 

I hereby declare that all information in this document has been obtained and presented in accordance with academic rules and ethical conduct. I also declare that, as required by these rules and conduct, I have fully cited and referenced all material and results that are not original to this work.

Name and Surname : Süleyman Özarslan

Signature 



\begin{abstract}
MobileCDP: A MOBILE FRAMEWORK FOR THE CONSUMER DECISION PROCESS

Özarslan, Süleyman

$\mathrm{PhD}$, Department of Information Systems

Supervisor: Assist. Prof. Dr. P. Erhan Eren
\end{abstract}

March 2015, 139 pages

Cognitive limitations of consumers decrease decision quality, and increase time spent as well as costs related to shopping. A widely accepted model covering consumer activities is the consumer decision process, which highlights five interrelated stages: problem recognition, information search, evaluation of alternatives, purchase, and post-purchase evaluation. Mobile information systems bring in significant capabilities for helping consumers deal with the challenges associated with all these stages. However, existing studies in the literature are limited in terms of quantity and functionality, and mostly restricted to the individual stages of the consumer decision process instead of the whole process. Accordingly, they have limited benefits for consumers. Since the stages are interrelated and the data collected in one stage are also valuable for another, we propose a mobile framework designed to provide assistance in all the stages of the Consumer Decision Process, named MobileCDP. It incorporates facilitating components corresponding to each stage as well as unifying components appearing across all stages. Quality and amount of the information are also important for making more accurate decisions, and some information cannot be gathered without participation of the consumers, such as information about grocery products. Consequently, we use participatory sensing approach to empower our framework. A prototype is also implemented and evaluated to show the applicability of the framework. The data collected in the experiments show that the functions provided by the prototype are useful, well integrated, and easy to use. Moreover, statistical analysis of the results indicates that the prototype reduces time, costs, and cognitive effort of the user.

Keywords: Consumer decision process, mobile information systems, participatory sensing 


\title{
öz
}

\section{MobileCDP: TÜKETICI KARAR SÜRECi IÇiN BIR MOBIL ÇERÇEVE}

\author{
Özarslan, Süleyman \\ Doktora, Bilişim Sistemleri Bölümü \\ Tez Yöneticisi: Yard. Doç. Dr. P. Erhan Eren
}

Mart 2015, 139 sayfa

\begin{abstract}
Tüketicilerin bilişsel sınırlamaları verdikleri kararların kalitesini düşürmekte ve alışveriş ile ilgili harcanan zamanı ve maliyeti artırmaktadır. Tüketici karar süreci, tüketici faaliyetlerini kapsayan ve yaygın kabul gören bir model olup birbiriyle ilişkili beş aşamadan oluşmaktadır: problemin fark edilmesi, bilgi araştırma, alternatiflerin değerlendirilmesi, satın alma ve satın alma sonrası değerlendirme. Mobil bilgi sistemleri, tüketicilerin bu aşamalar ile ilgili sorunlarını çözmek için önemli kabiliyetlere sahiptir. Ancak, literatürdeki mevcut çalışmalar miktar ve işlevsel açısından sınırlıdır ve çoğunlukla tüketici karar sürecinin bütünü yerine sürecin belirli aşamalarıyla kısıtlıdır. Bu nedenle, tüketiciler için sınırlı fayda sağlamaktadırlar. Aşamalar birbirleriyle ilişkili olduğundan ve bir aşamada toplanan veriler diğer aşamalar için de değerli olduğundan, MobileCDP isminde tüketici karar sürecinin bütün aşamalarında yardım sağlamak için tasarlanmış bir mobil çerçeve öneriyoruz. Bu çerçeve, her bir aşamaya karşılık gelen kolaylaştırıcı bileşenlerin yanı sıra birleştirici bileşenleri içermektedir. Bilginin kalitesi ve miktarı daha doğru kararlar vermek için önemlidir. Fakat, market ürünleriyle ilgili bilgiler gibi bazı bilgiler tüketicilerin katılımı olmadan toplanamamaktadır. Bu nedenle, önerdiğimiz çerçeveyi katılımcı algılama yaklaşımı ile güçlendirdik. Aynı zamanda, çerçevenin uygulanabilirliğini göstermek için bir prototip geliştirilmiş ve test edilmiştir. Testler sonucunda elde edilen veriler, prototip tarafından sağlanan fonksiyonların, kullanışı, tümleşik ve kullanımı kolay olduğunu göstermektedir. Ayrıca, sonuçların istatistiksel analizi, prototipin kullanıcının harcadığı zamanı, maliyetleri ve bilişsel çabayı azalttığını işaret etmektedir.
\end{abstract}

Anahtar Kelimeler: Tüketici karar süreci, mobil bilgi sistemleri, katılımcı algılama 
dedicated to my wife, son, and daughter, my parents, sister, and brother 


\section{ACKNOWLEDGEMENTS}

First and foremost, I would like to thank my advisor Dr. P. Erhan Eren for his inexhaustible support, guidance and patience throughout my research. I am very grateful for his inspiring ideas, he always provides insightful discussions about the research.

Also, I owe much to the thesis supervising committee members Dr. Altan Koçyiğit and Dr. İbrahim Körpeoğlu for helpful comments and discussions. I sincerely thank the examining committee members Dr. Nazife Baykal and Dr. Murat Perit Çakır for their suggestions and comments.

I also wish to thank my colleagues at the institute for an excellent working atmosphere. Special thanks to Serhat Peker, Oğuzhan Alaşehir, Bilgin Avenoğlu and Ebru Gökalp for their personal and scholarly interactions, and Sibel Gülnar for her support in administrative procedures.

I want to acknowledge TUBITAK (The Scientific and Technological Research Council of Turkey) and Scientific HR Development Program (OYP) for providing financial assistance during my PhD study.

Finally, I would like to express my very special gratitude to my family. I am very grateful to my beloved wife Nazife, for her endless love, patience and support during the thesis period. The birth of our girl Gülce was a great experience that we lived in this period. She adds a new dimension to our lives; and my son Mustafa, I will make up the lost time. I am very grateful to my parents, my sister Gamze and my brother Can for their endless love. 


\section{TABLE OF CONTENTS}

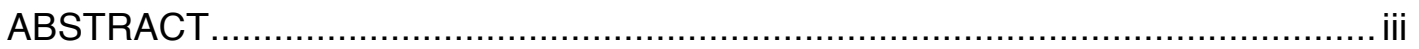

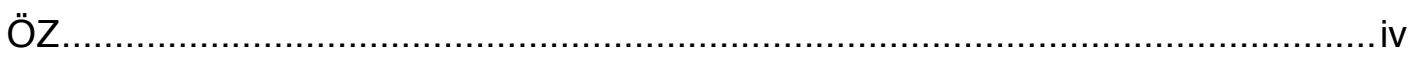

ACKNOWLEDGEMENTS .....................................................................

TABLE OF CONTENTS.......................................................................... vii

LIST OF TABLES .......................................................................................

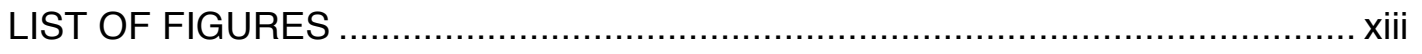

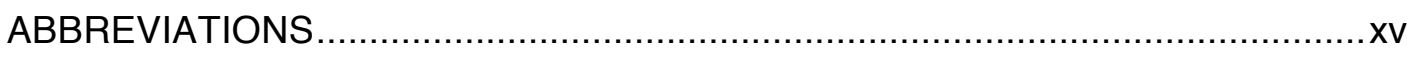

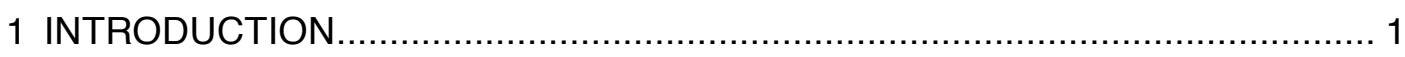

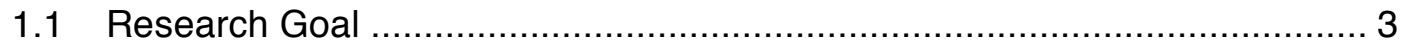

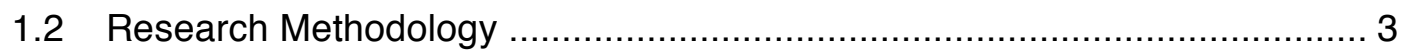

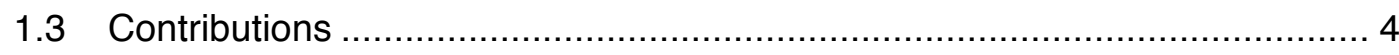

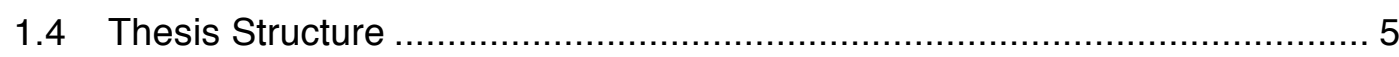

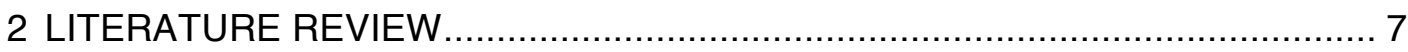

2.1 Consumer Decision Process .................................................................... 7

2.1.1 The Problem Recognition Stage .............................................. 9

2.1.2 The Information Search Stage ....................................................... 9

2.1.3 The Evaluation of Alternatives Stage ............................................... 10

2.1.4 The Purchase Stage .................................................................. 11

2.1.5 The Post-purchase Evaluation Stage ........................................... 11

2.1.6 Types of Consumers' Buying Behavior........................................ 11

2.1.7 Decision Rules Applied in the Consumer Decision Process ................ 12

2.1.8 Bounded Rationality, Satisficing Behavior, Heuristics, and Biases....... 13

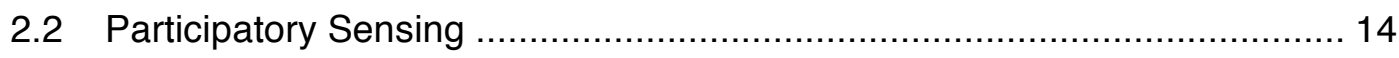

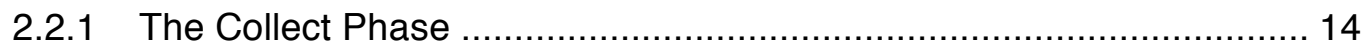

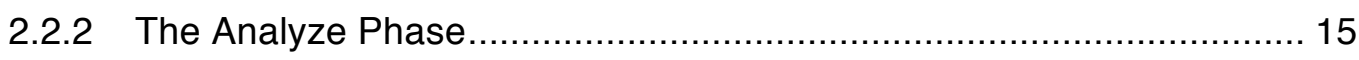

2.2.3 The Share Phase .......................................................................... 15

2.2.4 The Actuate Phase ......................................................................... 15

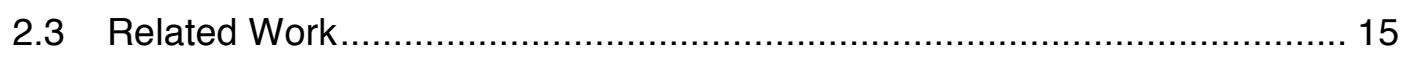


2.3.1 Consumer Decision Process Related Studies …............................... 16

2.3.2 Participatory Sensing Related Studies............................................. 19

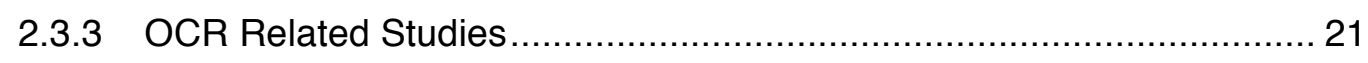

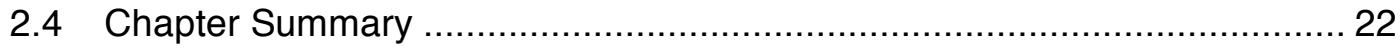

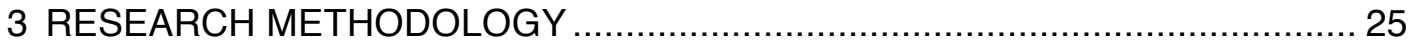

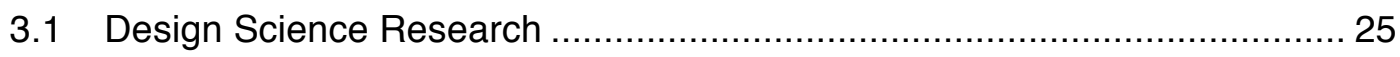

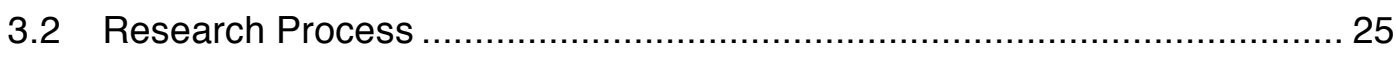

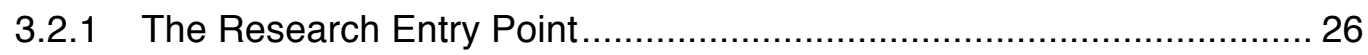

3.2.2 Activity 1: Identify Problem and Motivate ........................................... 26

3.2.3 Activity 2: Define Objectives of a Solution ...................................... 27

3.2.4 Activity 3: Design and Development ............................................... 27

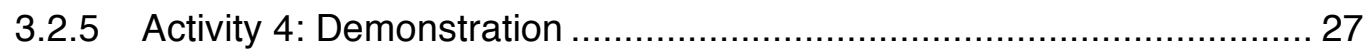

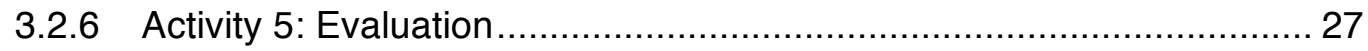

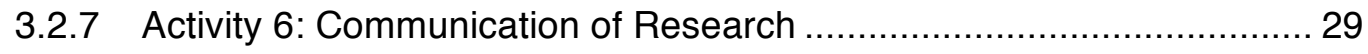

3.3 Validation of the Research Methodology .............................................. 29

3.3.1 Guideline 1: Design as an Artifact................................................. 29

3.3.2 Guideline 2: Problem Relevance ................................................... 31

3.3.3 Guideline 3: Design Evaluation ................................................ 31

3.3.4 Guideline 4: Research Contributions ............................................ 32

3.3.5 Guideline 5: Research Rigor .................................................. 32

3.3.6 Guideline 6: Design as a Search Process ...................................... 32

3.3.7 Guideline 7: Communication of Research......................................... 33

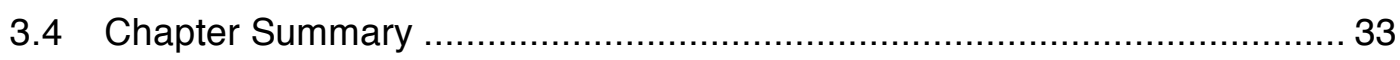

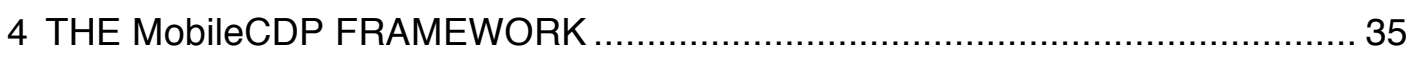

4.1 Components for the Problem Recognition Stage ...................................... 36

4.1.1 Context-aware Reminder Component ............................................... 37

4.1.2 Context-aware Personal Promotion Component .............................. 37

4.2 Components for the Information Search Stage .................................... 38

4.2.1 Experience Recorder Component................................................... 38

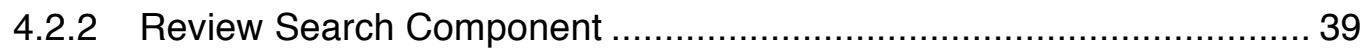

4.2.3 Information Aggregator Component.............................................. 39

4.3 Components for the Evaluation of Alternatives Stage ............................. 40

4.3.1 Multi-criteria Product Recommendation Component .......................... 40 
4.4 Components for the Purchase Stage .................................................... 41

4.4.1 Purchase Recommender Component.............................................. 42

4.5 Components for the Post-purchase Evaluation Stage ............................... 42

4.5.1 Post-purchase Review Component ................................................. 42

4.5.2 Post-purchase Feedback Component ........................................... 42

4.5.3 Post-purchase Consumer Support Component ................................ 42

4.6 Unifying Components ...................................................................... 43

4.6.1 Shopping List Component............................................................ 43

4.6.2 Data Collector Component.............................................................. 43

4.7 Extensibility of the MobileCDP Framework ............................................. 44

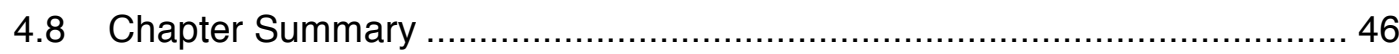

5 THE PARTICIPATORY SENSING APPROACH ..................................... 47

5.1 Participatory Sensing Support for the Components ................................. 48

5.1.1 Context-aware Reminder ............................................................ 48

5.1.2 Context-aware Personal Promotion ............................................... 48

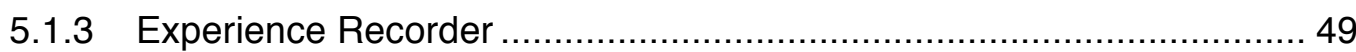

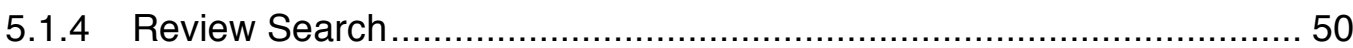

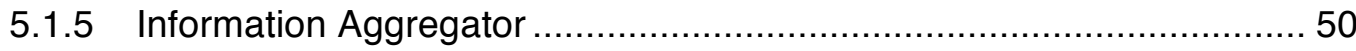

5.1.6 Multi-criteria Product Recommendation............................................ 50

5.1.7 Purchase Recommender ........................................................ 51

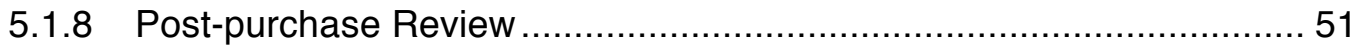

5.1.9 Post-purchase Feedback ............................................................ 52

5.1.10 Post-purchase Consumer Support................................................... 52

5.2 The Proposed Data Collection Method......................................................... 54

5.2.1 Image-Based Method ................................................................ 56

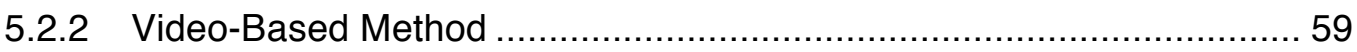

5.3 Proposed Multi-Criteria Product Recommendation Method ........................63 63

5.3.1 Inputs of the Proposed Method .......................................................... 63

5.3.2 Data Collection for Multi-criteria Recommendation............................. 65

5.3.3 Multi-criteria Decision Algorithms ................................................ 67

5.3.4 Implementation of the Proposed Method .......................................... 69

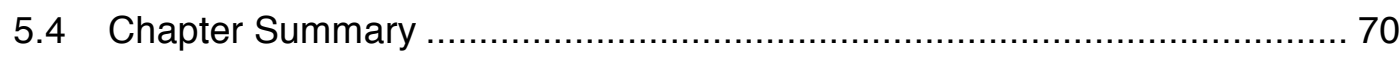

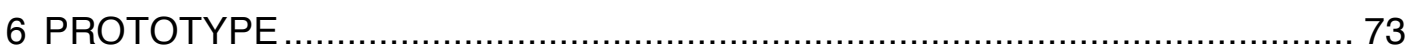




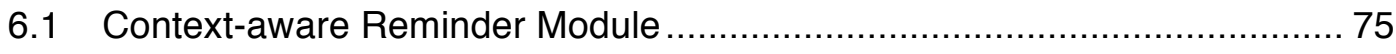

6.2 Context-aware Personal Promotion Module ................................................ 75

6.3 Experience Recorder Module ................................................................ 75

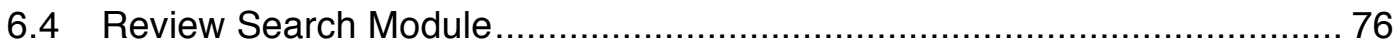

6.5 Information Aggregator Module ..................................................... 76

6.6 Multi-Criteria Product Recommender Module........................................ 77

6.7 Purchase Recommender Module ......................................................... 78

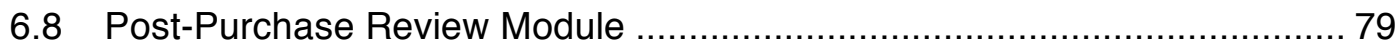

6.9 Post-purchase Feedback Module ........................................................... 79

6.10 Post-purchase Consumer Support Module .......................................... 80

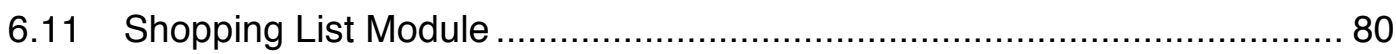

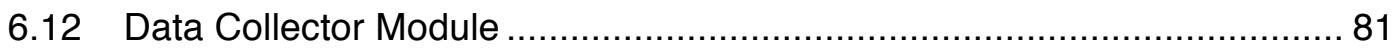

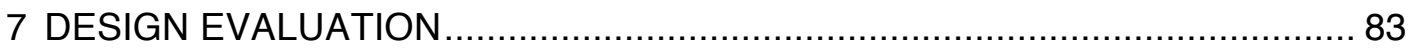

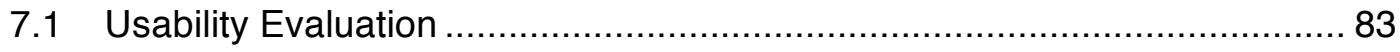

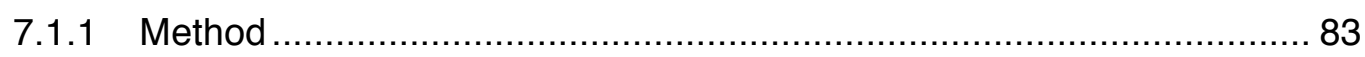

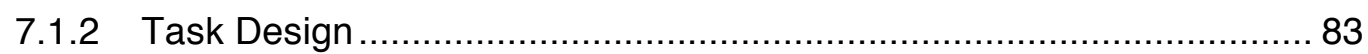

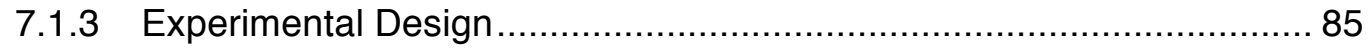

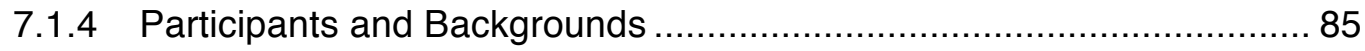

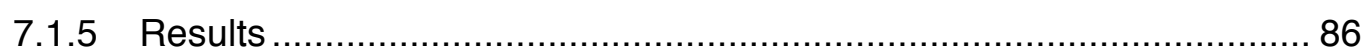

7.1.6 Summary of the Usability Evaluation .............................................. 89

7.2 Accuracy Evaluation of the Proposed Data Collection Methods................. 89

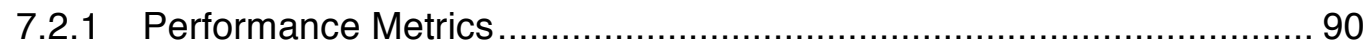

7.2.2 Experiments for the Image-Based Data Collection Method .................. 91

7.2.3 Experiments for the Video-Based Data Collection Method................... 92

7.3 Performance Evaluation of the Image-Based Data Collection Method........ 97

7.3.1 Phases of the Converting a Captured Image into Text ....................... 97

7.3.2 Measuring the Speed Performance ............................................... 98

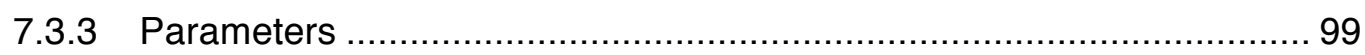

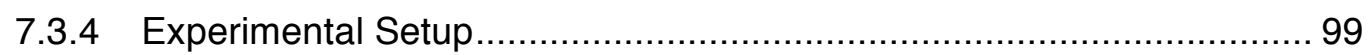

7.3.5 Comparing Speed Performance ................................................. 100

7.3.6 Comparing Accuracy Performance ................................................. 104

7.4 Multi-criteria Product Recommendation Experiments ............................ 106

7.4.1 Results of the Single Criterion Ranking Algorithm .......................... 108 
7.4.2 Results of the Weighted Sum Algorithm ...................................... 108

7.4.3 Results of the ELECTRE III Algorithm .......................................... 109

7.4.4 Results of the PROMETHEE II Algorithm ....................................... 110

7.4.5 Discussion of the Results of the Decision Making Algorithms ........... 110

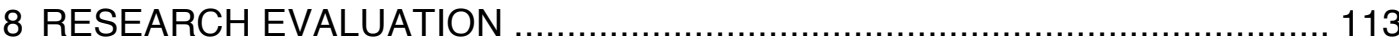

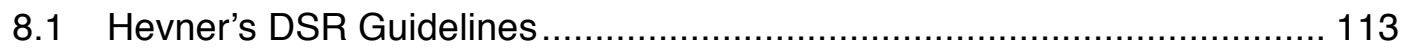

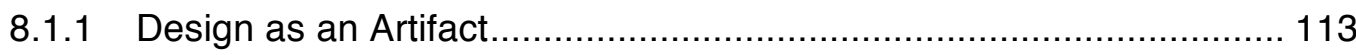

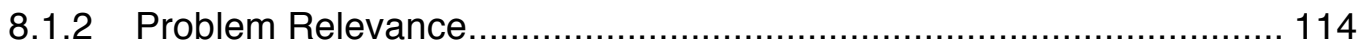

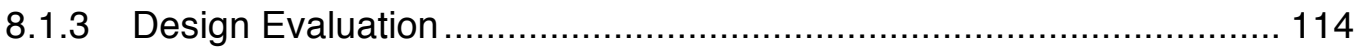

8.1.4 Research Contributions ............................................................. 114

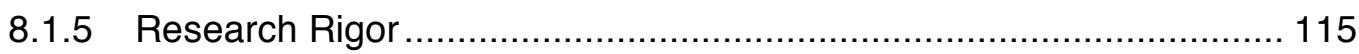

8.1.6 Design as a Search Process .......................................................... 116

8.1.7 Communication of Research........................................................... 117

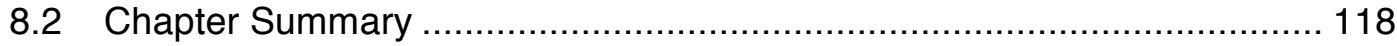

9 CONCLUSION AND FUTURE RESEARCH .......................................... 119

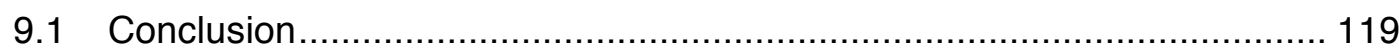

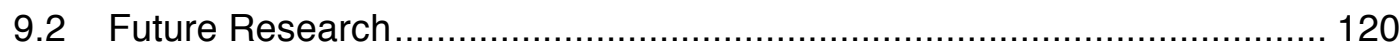

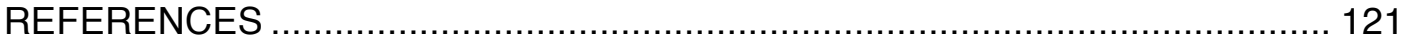

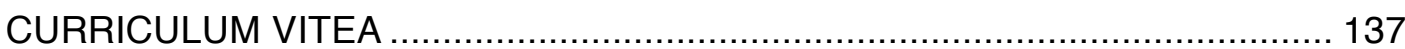




\section{LIST OF TABLES}

Table 2.1 Consumers' activities and challenges in each stage of the consumer

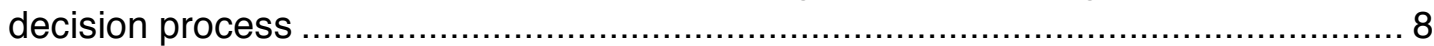

Table 2.2 Studies related to the individual stages of the CDP ............................ 17

Table 3.1 Design science research evaluation methods (adapted from [25]) ........... 28

Table 3.2 DSR publication schema (adapted from Gregor and Hevner [27]) ........... 30

Table 3.3 Design science research guidelines (adapted from Hevner, et al. [25])... 31

Table 4.1 The proposed components and justifying studies in the literature ............ 36

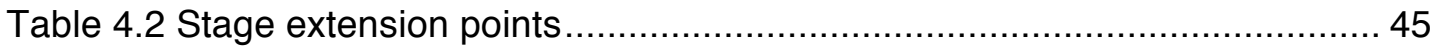

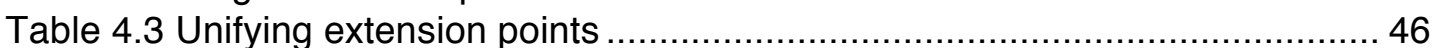

Table 5.1 Components of the MobileCDP framework regarding the participatory

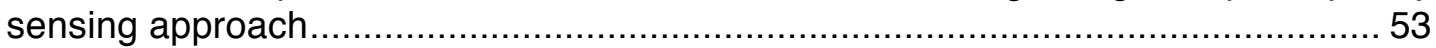

Table 5.2 Frequently encountered OCR errors ............................................ 58

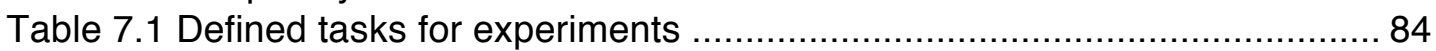

Table 7.2 Descriptive statistics and t-test results (seconds) ................................8 88

Table 7.3 Percentage of number of unsuccessful tasks due to selection errors...... 89

Table 7.4 Word and character recognition rates of the image based method ......... 92

Table 7.5 Word and character recognition rates of individual video sequences ...... 95

Table 7.6 Evaluative criteria, criteria weights, and preference directions ............. 106

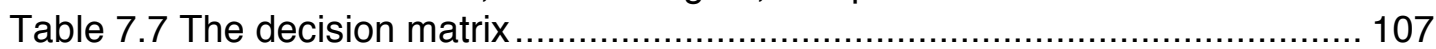

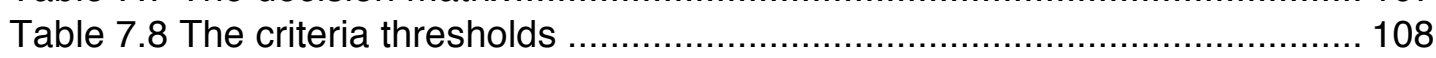

Table 7.9 The results of the single criterion ranking algorithm........................... 108

Table 7.10 The results of the weighted sum algorithm ...................................... 109

Table 7.11 The results of the ELECTRE III algorithm ......................................... 109

Table 7.12 Results of the PROMETHEE II algorithm ...................................... 110

Table 7.13 Used data by each algorithm ...................................................... 111

Table 7.14 Comparison of the results of the decision algorithms ........................ 111

Table 8.1 Mapping thesis chapters to the DSRM process of Peffers, et al. [26] ... 117 


\section{LIST OF FIGURES}

Figure 3.1 Design science research methodology process model (adapted from Peffers, et al. [26])......................................................................................... 26

Figure 4.1 Components of the MobileCDP framework....................................... 35

Figure 4.2 Data exchange among the components of the MobileCDP framework .. 37

Figure 5.1 OCR and KBC processes applied to images for image-based method .. 57

Figure 5.2 The proposed video-based approach ................................................ 59

Figure 5.3 (a) A sample frame extracted from a video sequence (b) The OCR output

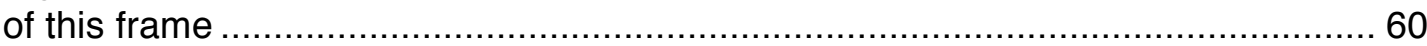

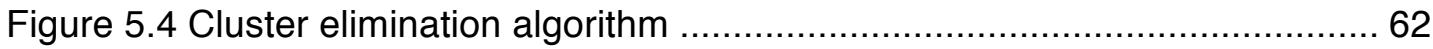

Figure 5.5 Implementation of the proposed multi-criteria recommendation method 70

Figure 6.1 Client-server architecture of the prototype......................................... 73

Figure 6.2 Consumer activities in a sample scenario and corresponding modules in

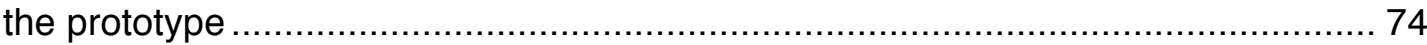

Figure 6.3 The screens of the Experience Recorder module: (a) My Purchases, (b) My reviews, and (c) My Feedbacks ............................................................. 76

Figure 6.4 The screens of the Information Aggregator module: (a) Product Details, and (b) Product List.................................................................................... 77

Figure 6.5 (a) Product alternatives screen (b) Product alternatives screen after

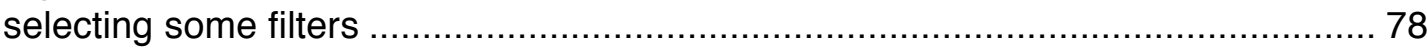

Figure 6.6 Sample screens of the purchase recommender module ...................... 79

Figure 6.7 (a) Add review (b) Send feedback (c) Shopping list.............................. 80

Figure 7.1 Histogram of the SUS scores of the participants ................................. 86

Figure 7.2 Result-set 1 from the SUS questionnaire (averages, higher rating is better, and error bars indicate standard deviations) ........................................... 87

Figure 7.3 Result-set 2 from the SUS questionnaire (averages, lower rating is better, and error bars indicate standard deviations) ..................................................... 87 Figure 7.4 Average Task Completion Time (TCT) for each task in the no-prototype

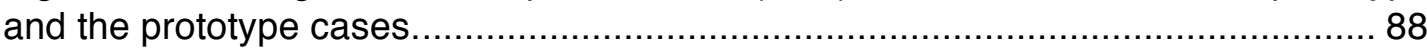

Figure 7.5 A sample receipt captured by the mobile phone camera...................... 91

Figure 7.6 A sample frame of a captured video ................................................. 93

Figure 7.7 Comparison of the proposed TB-MFI method with Super Resolution and

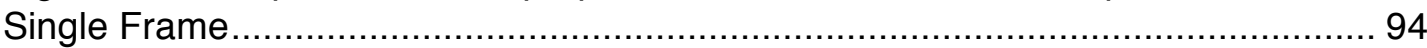

Figure 7.8 Word and character recognition rates versus the number of frames ...... 96 Figure 7.9 (a) Total execution time of the in-phone approach (b) Total execution time of the remote server approach ....................................................................... 101

Figure 7.10 (a) Total execution time for $1536 \times 2048$ pixel resolution (b) Total execution time for 768x1024 pixel resolution .................................................... 102

Figure 7.11 Total execution time for $384 \times 512$ pixel resolution............................. 102 
Figure 7.12 (a) OCR time for in-phone approach (b) OCR time for remote server approach ..... 102

Figure 7.13 OCR time for the in-phone and the remote server approaches in $768 \times 1024$ pixel resolution 103

Figure 7.14 Network delay for the remote-server approach............................... 103

Figure 7.15 Compression and decoding delay of compressed images ................. 104

Figure 7.16 (a) WRR and CRR for varied resolutions (b) WRR and CRR for varied

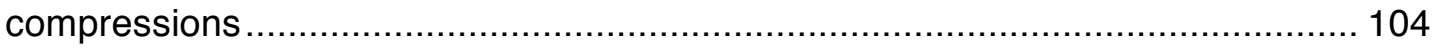

Figure 8.1 The DSRM process (adapted from [26]) …..................................... 115

Figure 8.2 The DSRM Process of this research............................................ 116 


\section{ABBREVIATIONS}

100BASETX: 100-Mbps Baseband Twisted Pair

3D: 3 Dimensional

ADSL: Asymmetric Digital Subscriber Line

AHP: Analytic Hierarchy Process

ANOVA: ANalysis Of VAriance

API: Application Program Interface

AVC: Advanced Video Coding

CDD: Compression and Decoding Delay

CDP: Consumer Decision Process

CPU: Central Processing Unit

CRR: Character Recognition Rate

df: Degrees of Freedom

DSR: Design Science Research

DSS: Decision Support Systems

DSRM: Design Science Research Methodology

EKB: Engel, Kollat, and Blackwell

ELECTRE: ELimination and Choice Translating Reality

GDSS: Group Decision Support Systems

GHz: Gigahertz

GIS: Geographical Information System

GPS: Global Positioning System 
GSM: Global System for Mobile

GUI: Graphical User Interface

HDD: Hard Disk Drive

HR: High Resolution

IRE: Information Requirement Elicitation

IS: Information Systems

ISO: International Organization for Standardization

IT: Information Technologies

JPEG: Joint Photographic Experts Group

KBC: Knowledge Based Correction

LR: Low Resolution

M: Mean

Mbps: Megabits per Second

MCDA: Multi-criteria Decision Analysis

MCDM: Multi-criteria Decision Making

MISQ: Management Information Systems Quarterly

MPEG4: Moving Picture Experts Group 4

ms: Millisecond

ND: Network Delay

NFC: Near Field Communication

NORS: Nokia Remote Sensing

OCR: Optical Character Recognition

OS: Operating System

PEIR: Personal Environmental Impact Report

PRISM: Platform for Remote Sensing using Smartphones 
PROMETHEE: Preference Ranking Organization Method for Enrichment Evaluations

PS: Participatory Sensing

Q-factor: Quality Factor

RADP-VPC: Reverse Auction Dynamic Price with Virtual Participation Credit

RAM: Random Access Memory

SD: Standard Deviation

Sig.: Significance

SR: Super Resolution

Std.Dev: Standard Deviation

SUS: System Usability Score

TB-MFI: Text Clustering Based Multi-Frame Integration

RFID: Radio Frequency Identification

TCT: Task Completion Time

TET: Total Execution Time

TL: Turkish Lira

TV: Total Variation

UPC: Universal Product Code

WOM: Word of Mouth

WRR: Word Recognition Rate

XMCDA: XML Multi-Criteria Decision Aid

XML: Extensible Markup Language 





\section{CHAPTER 1}

\section{INTRODUCTION}

Consumers experience various difficulties as part of their activities due to cognitive limitations of human beings. Accordingly, consumer behavior research addresses challenges in consumer activities, such as how consumers select, use, and dispose of products in order to satisfy their needs [1]. Bettman, et al. [2] define consumers as "limited information processors", and these limitations consist of limited computational capacities and limited memory. Consequently, consumers cannot process a high amount of information, such as they can consider a maximum of five products and six attributes of the products while making a decision among various product alternatives [2-5]. In addition, consumers' limited capacity of calling information from memory prevents perfectly recalling a long shopping list, past purchases, and relevant product information [6]. Overall, cognitive limitations of consumers decrease the probability of identifying the optimal choice, and increase time spent, costs, and cognitive effort related to shopping [7].

In order to aid consumers in their activities, similar to other application domains such as health and tourism [8, 9], mobile information systems provide significant capabilities for reducing cognitive effort, time and cost, as well as increasing the quality of the decision. However, studies related to the use of mobile information systems in the consumer domain are rather limited in scope. These studies primarily focus on providing assistance with regard to specific tasks such as price comparison and product review [10-12]. This situation suggests an unexplored potential in providing improved benefits to the consumer by supporting complementary functionalities as part of a unified approach.

In fact, consumer behavior research introduces and advocates such a unified approach. Various models have been developed in the literature in order to describe the consumer behavior. The Nicosia model [13], Howard-Sheth model [14], and EKB (Engel, Kollat, and Blackwell) model [15] are referred to as "grand models" of the consumer behavior literature [16, 17]. All these models have highlighted the Consumer Decision Process (CDP), which consists of consumers' entire activities in five stages, as the most important part of the consumer behavior. These stages are the problem recognition, information search, evaluation of alternatives, purchase decision, and post-purchase evaluation [1, 4, 18]. 
The problem recognition stage occurs when a consumer identifies a problem or need, and what kind of product can satisfy this need [5]. After a need is recognized, the consumer passes to the second stage referred to as the information search. In this stage, the consumer collects information about the product category, alternatives and brands [8]. Next, the consumer compares product alternatives in the third stage, the evaluation of alternatives [10]. Once the consumer evaluates the alternatives, he or she chooses a particular product and brand in the fourth stage, the purchase decision [8]. The post-purchase evaluation is the final stage, where the customer evaluates whether he or she is satisfied or dissatisfied with the purchased product.

Although mobile information systems provide significant capabilities to address challenges of consumers in the consumer decision process, to the best of our knowledge and according to a recent research study [19], there is currently no study exploring a holistic approach supporting consumers on all stages of the consumer decision process. Existing studies focus on individual tasks such as product review, price comparison, shopping lists; therefore, a comprehensive solution is needed to support all stages of the consumer decision process.

One of the main obstacles of such a solution is collecting the required data for each stage of the process; for example, prices and features of grocery items which consumers do not typically purchase online. The Participatory Sensing (PS) approach addresses this challenge by enabling collection of the required data through the contributions of participants, and allowing participants to gather, use and share information collected by their mobile devices [20]. Typical participatory sensing applications run in a centralized fashion and include four phases: collect, analyze, share and act [21]. In the collect phase, participants collect data by their mobile phones [20]. Then, in the analyze phase, the collected data are transmitted to a central server to extract meaningful information by using simple statistical techniques as well as complex methods such as machine-learning techniques. Next, in the share phase, analyzed data are made available in various forms, such as visualization on mobile applications, web portals, and social networks depending on the application needs $[22,23]$. Finally, the output of the participatory sensing system activates an actuation agent (e.g., a person [11], a group of people [23] or another system [22]) in the actuate phase. For instance, the system generates and sends a reminder message to a person, then he or she buys the products mentioned in the message.

This research is focused on investigating consumers' challenges in their activities by examining literature on the consumer behavior domain, and addressing these challenges through the use of mobile technologies, specifically the mobile information systems and the participatory sensing approach. Accordingly, this research applies theory from a social science domain to solve problems of consumers using mobile technologies. 


\subsection{Research Goal}

The aim of this study is to propose a mobile framework, MobileCDP, addressing the challenges of consumers' activities by following a unified approach as advocated by the consumer decision process literature. The stages of the consumer decision process are taken as a foundation for the framework, and the articulated components of MobileCDP are based on the reported findings in the literature. Therefore, MobileCDP is intended to become a holistic and extensible solution to assist consumers in all stages of the consumer decision process. In addition, we integrate the participatory sensing approach to the MobileCDP framework to address the data collection challenge. Since the proposed framework is a holistic solution, it enables collecting and using information in each stage of the decision process. Therefore, it can collect and utilize more information than individual solutions developed for different stages of the consumer decision process, such as price comparison and product review systems. Moreover, by integrating the participatory sensing approach; information is collected, used and shared between consumers in a participatory way, which increases the amount and quality of information, and enables making more efficient and effective decisions.

The MobileCDP framework proposes solutions for consumers' challenges in each stage of the consumer decision process. For example, MobileCDP prevents forgetting of purchasing needed product and helps to create required stimuli for the problem recognition stage by sending reminder messages and creating shopping lists. It also assists consumers in the information search stage by facilitating internal and external search activities. MobileCDP records consumers' purchases and information search activities, and helps recall information later to address the challenge of cognitive limitation for recalling information from the memory. It also assists external information search by collecting information from external sources, such as online resources. The proposed framework also makes recommendations to consumers to facilitate the evaluation of alternatives stage by decreasing the cognitive effort required for the decision, reducing the time for evaluating alternatives, and improving consumers' decision quality. In order to support the purchase decision stage of the consumer decision process, MobileCDP provides recommendations by combining information on product prices, stores, campaigns and discounts through the use of credit or store cards. It also assists consumers in the post-purchase evaluation stage by sharing complaints, satisfactions, suggestions and reviews on a product with stores and product manufacturers.

\subsection{Research Methodology}

In this research, we used the Design Science Research (DSR) paradigm as the research methodology, which is accepted as a legitimate approach for Information Systems (IS) research [24-28]. We follow Hevner's DSR guidelines [25] to satisfy the requirements of an effective design science research. 
In addition to these guidelines, we follow Design Science Research Methodology (DSRM) Process Model suggested by Peffers, et al. [26], which covers the entire research from motivation to communication. In order to construct this PhD thesis, we use the Design Science Research Publication Schema proposed by Gregor and Hevner [27] for design science research projects.

\subsection{Contributions}

In this section, we present the three main contributions of this research: the framework, the proposed integrated and holistic approach, the algorithms developed for automated data collection, and the prototype and its evaluation.

\section{Contribution 1 (the MobileCDP framework):}

The primary contribution of this research is the artifact, the proposed MobileCDP framework. As explained in Chapter 2.3.1, existing studies in the literature focus on specific stages of the consumer decision process, such as information search or evaluation of alternatives. According to the best of our knowledge and consistent with recent research [19], MobileCDP is the first study based on an integrated and holistic approach addressing all stages of the consumer decision process. The integrated and holistic approach provided by this research contributes to the body of knowledge in the area of mobile information systems for consumers. This approach includes the facilitating components that assist a stage of the consumer decision process as explained in Chapter 4, and the unifying components that integrate and coordinate the facilitating components as described in Chapter 4.6. The evaluation of the developed prototype shows that such an approach provides benefits to consumers by enabling utilization of more information compared to stand-alone solutions developed for individual stages. According to the results of the evaluation (Chapter 7), the proposed MobileCDP framework reduces cognitive effort, time and cost, and increases the quality of the decision.

\section{Contribution 2 (the algorithms for automated data collection):}

As mentioned before, a key issue is collecting the required data for each stage of the process. Accordingly, we also propose a solution to provide an automated mechanism for data collection. Briefly, this solution extracts information from store receipt images and videos taken by mobile devices of consumers, and eliminates errors in the extracted information. A Text Clustering Based Multi-frame Integration algorithm (TB-MFI) is developed to integrate information in the video frames in order to improve the data recognition accuracy, and a Knowledge Based Correction (KBC) algorithm is developed in support of the Optical Character Recognition (OCR) to correct inaccurately recognized information. 


\section{Contribution 3 (the prototype and evaluation):}

In addition to the design of MobileCDP, a prototype is implemented to demonstrate the applicability of the proposed approach. According to Hevner, et al. [25], as an instantiation of the primary artifact, the prototype implementation is also a contribution. In addition, experiments are carried out on the prototype in order to evaluate the provided benefits as well as the usability aspects.

\subsection{Thesis Structure}

As mentioned above, the Design Science Research Publication Schema [27] is followed to build the structure of the thesis, and the Design Science Research Process Model [26] is followed to construct this research. These models are explained in Chapter 3, in detail.

The rest of the thesis is organized as follows:

Chapter 2 presents the literature review and provides the respective knowledge base for this research. Particularly, this chapter covers descriptions of the stages of the consumer decision process, definitions of the phases of the participatory sensing approach, and a brief summary of related studies and the corresponding stages they target.

Chapter 3 introduces the research methodology used in this research, the Design Science Research paradigm, and explains conformity of this research to this research methodology.

Chapter 4 describes the components of the proposed MobileCDP framework as well as justifications of these components based on the relevant literature, and how the framework supports consumers using these components.

Chapter 5 presents benefits of the participatory sensing approach for the MobileCDP framework as well as descriptions of each component in terms of the phases of the participatory sensing approach.

Chapter 6 describes the implemented prototype mobile application that is based on the MobileCDP framework.

Chapter 7 discusses the qualitative and quantitative evaluation of the prototype.

Chapter 8 evaluates this research against the Design Science Research (DSR) guidelines of Hevner, et al. [25].

Chapter 9 provides concluding remarks and suggestions about future research. 


\section{CHAPTER 2}

\section{LITERATURE REVIEW}

Two main areas in the literature are relevant to the research presented in this study: the consumer decision process and the participatory sensing approach. This chapter has been divided into three parts. The first part describes the stages of the consumer decision process as well as consumers' challenges in these stages, consumers' buying behavior, and decision rules applied in the consumer decision process. Then, a brief overview of the participatory sensing approach is given in the second part. The final part presents related work in the literature, which is divided into the consumer decision process related studies, the participatory sensing related studies, and the optical character recognition (OCR) related studies.

\subsection{Consumer Decision Process}

In this section, first, consumer behavior and the consumer decision process are defined, and each stage of the consumer decision process is described with consumers' challenges. Then, consumers' buying behavior and decision rules are explained to clarify the consumer decision process.

According to Engel, et al. [4], consumer behavior is "activities directly involved in obtaining, consuming, and disposing of products and services, including the decision processes that precede and follow these actions". Dibb [29] also states that consumer behavior includes both the act of the purchase itself and pre/post purchase activities.

Researchers develop different models in order to explain the consumer behavior [1, $4,18]$. According to the common ground of these models, the five-stage consumer decision process is the major part of the consumer behavior, and includes the problem recognition, information search, evaluation of alternatives, purchase decision, and post-purchase evaluation stages $[1,4,18]$.

Table 2.1 summarizes consumers' activities and challenges in each stage of the consumer decision process. 
Table 2.1 Consumers' activities and challenges in each stage of the consumer decision process

\begin{tabular}{|c|c|c|}
\hline Stage & Activities & Challenges \\
\hline $\begin{array}{l}\text { Problem } \\
\text { Recognition }\end{array}$ & $\begin{array}{l}\text { - Internal stimuli (e.g., hunger, } \\
\text { thirst) } \\
\text { - External stimuli (e.g., } \\
\text { advertisings, marketing } \\
\text { efforts) }\end{array}$ & $\begin{array}{ll}- & \text { Limited capacity of } \\
\text { calling information from } \\
\text { memory } \\
\text { - } & \text { Creating required stimuli }\end{array}$ \\
\hline Information Search & $\begin{array}{ll}\text { - } & \text { Internal search (e.g., } \\
\text { searching product } \\
\text { information from memory) } \\
\text { - } \quad \text { External Search (e.g., social } \\
\text { networks, product websites) }\end{array}$ & $\begin{array}{l}\text { - Limited computational } \\
\text { capacity to process } \\
\text { information } \\
\text { - Finding information } \\
\text { about grocery products } \\
\text { - Information overload }\end{array}$ \\
\hline $\begin{array}{l}\text { Evaluation of } \\
\text { Alternatives }\end{array}$ & $\begin{array}{l}\text { Determining the evaluative } \\
\text { criteria } \\
\text { - Deciding alternatives to } \\
\text { evaluate } \\
\text { - Valuing the performances of } \\
\text { the alternatives } \\
\text { - Applying a decision rule }\end{array}$ & $\begin{array}{l}\text { - } \quad \text { Limited capacity of the } \\
\text { working memory to } \\
\text { consider multiple } \\
\text { product alternatives and } \\
\text { criteria } \\
\text { - Limited computational } \\
\text { capacity to use complex } \\
\text { decision rules }\end{array}$ \\
\hline Purchase & $\begin{array}{l}\text { Five purchase sub- } \\
\text { decisions: Brand, vendor, } \\
\text { quantity, timing, and } \\
\text { payment-method }\end{array}$ & $\begin{array}{l}\text { Limited computational } \\
\text { capacity and working } \\
\text { memory to consider } \\
\text { purchase sub-decisions }\end{array}$ \\
\hline $\begin{array}{l}\text { Post-Purchase } \\
\text { Evaluation }\end{array}$ & $\begin{array}{l}\text { - } \text { Making a complaint } \\
\text { - Purchasing the product } \\
\text { again } \\
\text { Talking favorably or } \\
\text { unfavorably about the } \\
\text { product with other } \\
\text { consumers }\end{array}$ & $\begin{array}{l}\text { Sharing post-purchase } \\
\text { evaluations with related } \\
\text { sides (e.g., other } \\
\text { consumers, product } \\
\text { manufacturers, vendors } \\
\text { and stores) }\end{array}$ \\
\hline
\end{tabular}




\subsubsection{The Problem Recognition Stage}

Problem recognition is the difference between the consumer's desired (ideal) state and the actual situation. In the problem recognition stage, the consumer perceives a need, recognizes the problem, and becomes motivated to solve the problem that he or she has just recognized. An internal or external stimulus can trigger the problem recognition.

An internal stimulus is the consumer's personal perception, such as feeling hunger, and deciding to order a meal. However, an external stimulus is an outside influence, such as seeing a store advertising for a notebook, and deciding to purchase a new notebook.

In this stage, the consumer records needed product to his or her memory after encountering an internal or external stimulus, but they can forget to purchase the needed product because of the limited long-term memory of the consumer [4].

\subsubsection{The Information Search Stage}

After an awareness of the problem has been built, the consumer passes to the second stage of the consumer decision making process: information search. Engel, et al. [4] describe this stage as "the motivated activation of knowledge stored in memory or acquisition of information from the environment". Therefore, information search can be either internal or external.

In internal search, a consumer searches their memory for information about products based on past experience of the product, information obtained from past marketing advertisements, and information collected from the word of mouth (WOM) recommendations in memory. On the other hand, consumers have cognitive limitations for recalling information from memory [2].

Internal search is generally sufficient for frequently purchased products, while it is insufficient for the following conditions: when past experience is inadequate, when there is a high risk situation for making a wrong purchase decision, and when the cost of gathering additional external information is low.

Above conditions trigger external information search. In external search, a consumer searches additional information focused on personal sources such as friends, public sources such as social networks, and marketer dominated sources, such as company websites. Although information about (e.g., price, features) some types of products, such as electronic devices, can be easily found using online resources; it is difficult to find information about grocery products, which are not generally purchased over the Internet.

Consumers face greater memory constraints in environments with higher levels of information [30]. The current environment of consumers includes information overload, and a vast amount of product alternatives. A typical grocery store includes 
more than 30000 products [31]. The cost of processing information increases as the decision complexity rises [32].

\subsubsection{The Evaluation of Alternatives Stage}

The consumer evaluates alternatives in his/her evoked set in order to make a choice at this stage. According to Engel, et al. [4], four tasks are involved in this stage:

Task 1 - The consumer must determine the evaluative criteria to use for the purchase:

Evaluative criteria reflect features or characteristics that the consumer wants or does not want, and presents the objective attributes of a brand (such as the CPU capacity of a mobile phone) and the subjective factors (such as prestige). Evaluative criteria may differ in their importance or salience. For example, the price is the most important criterion in some decisions, while the quality is the most important one in some other decisions. The consumer can use a maximum of six evaluative criteria because of the cognitive limitations of consumers $[3,7]$.

Task 2 - The consumer must decide alternatives to evaluate:

In addition to evaluative criteria, consumers must also determine the set of alternatives, the evoked set. If the consumer has sufficient prior knowledge to choose alternatives, he or she can recall them from his or her memory. Otherwise, the consumer looks to the environment for assistance in forming his or her evoked set [4]. Although the average number of known brands generally varies between ten and twenty according to the product type, consumers can consider only three to five product alternatives [9]. The increasing complexity of the decision and the increasing number of product alternatives compromise the consumer's ability to efficiently make good decisions [33].

Task 3 - The consumer must value the performance of alternatives:

Once consumers choose alternative products (the evoked set), they evaluate performances of these products regarding the evaluative criteria determined in the first task. Consumers use their existing knowledge to judge the performance of choice alternatives in their evoked set along determined evaluative criteria. When the existing knowledge is not sufficient, an external search must be required to judge the performance of alternatives. In addition, certain cues or signals may be used to make judgments. For example, the price is commonly used to infer product quality [4].

Task 4: The consumer must apply a decision rule to make the final product choice:

Finally, consumers apply decision rules (procedures and strategies) to decide the final product. These decision rules can be retrieved from memory, or consumers can build constructive decision rules to fit situational contingencies [4]. Decision rules 
have variable complexity. Simple decision rules generally have one attribute; for example, consumers buy what they bought last time. Complex decision rules have multiple-attributes.

\subsubsection{The Purchase Stage}

The outcome of the evaluation of alternatives stage is an intention to purchase: buy or do not buy. Once consumers evaluate the alternatives, they decide a particular product and brand in the fourth stage of the consumer decision process named as the purchase stage [4]. According to Assael [34], usually this product has the most adequate performance regarding the evaluative criteria. Consumers make up to five purchase sub-decisions to decide for a purchase [34]. These are brand, vendor, quantity, timing, and payment-method decisions.

\subsubsection{The Post-purchase Evaluation Stage}

Post-purchase evaluation is the final stage, where consumers evaluate whether they are satisfied or dissatisfied with the purchased product. After purchasing the product, consumers begin to evaluate the performance of the product in the process of consumption. The outcome of the evaluation is satisfaction or dissatisfaction. It relies on the relationship between expectations of the consumer and perceived performance of the product. According to Kotler [35], if the product meets expectations, the consumer is satisfied; otherwise, the consumer is dissatisfied. In addition, according to Dibb [29], post-purchase evaluation determines whether the consumer makes a complaint, purchases the product again, and talks favorably or unfavorably about the product with other consumers.

\subsubsection{Types of Consumers' Buying Behavior}

The consumer decision process depends on the type of the product [36]. For example, there are differences among degrees of involvement for purchasing a toothpaste, a laptop, and a new car. It is related to the complexity of the decision; more complex decisions require more involvement of consumers, and vice versa. $A$ common way to characterize the decision making process is considering the amount of the effort spent for the decision making [36]. Briefly, there are three types of buying behavior: the extended problem solving, the limited problem solving, and the routinized problem solving.

The first type of consumers' buying behavior is the extended problem solving. In this type, the decision carries high risk and requires high involvement of the consumer. The consumer is unfamiliar with the product class, product alternatives, and criteria to consider for selecting the product. The consumer extensively searches for information, and uses multiple information sources consulted prior to store visits. He or she carefully evaluates each product alternative, and considers multiple criteria. Therefore, the consumer spends considerable time for information search and evaluation of alternatives for extended problem solving. 
The second type is the limited problem solving. This buying behavior is less complex than the first one, and usually simpler and straightforward. Consumers consider several product alternatives and only the most prominent criteria. In addition, they make a limited search about products and in-store decisions. The consumer involvement level is medium in this buying behavior.

The third type is the routinized problem solving, which is the simplest one. Consumers apply this behavior for purchasing low cost and frequently purchased products, since they do not want to spend a lot of time to make the purchase. This type of buying behavior consists of limited information search and minimal alternative evaluation. The consumer involvement is low in routinized buying behavior.

\subsubsection{Decision Rules Applied in the Consumer Decision Process}

As mentioned in Chapter 2.1.3, the last task of the evaluation of alternatives stage is applying a decision rule to make the final product choice. Decision rules are divided into two categories: compensatory and non-compensatory decision rules [36]. Simple decisions are non-compensatory, which eliminate all options that do not meet basic standards [36]. For example, even if it was superior to existing brands, a new brand would not be considered according to a non-compensatory decision rule such as "only buy well-known brands". Therefore, non-compensatory rules decrease the possibility of the selecting a considerably good product. Compensatory decision rules give a chance to the product to cover its disadvantages [36]. According to compensatory rules, the consumer evaluates product alternatives in terms of determined criteria, and computes an overall score for each product, then selects the product with the highest score. There are two main types of compensatory rules applied by consumers: the single additive rule and the weighted sum rule. The weighted sum rule is more complex, and implies consideration of the relative importance of the rated criteria. A high-scored criterion of the product can cover a low-scored criterion in the weighted sum method. However, because of the cognitive limitations of consumers to process information, they cannot use complex decision rules like the weighted sum rule to establish optimal choice [6].

Recommendation systems help consumers by recommending relevant products. These systems filter collected information by applying some data analysis techniques, then recommend products to feed the evaluation of alternatives stage with a list of the recommended products.

According to Changchien, et al. [37], today's recommendation systems can be categorized into two categories:

Content Based Filtering: The content based filtering method provides items that are similar to consumers' past purchased items. This method creates a consumer profile according to past purchases of the consumer, and provides a recommended product list using conformity of the products to the profile. As a disadvantage, different or 
totally unique products cannot be recommended by the content based filtering method.

Collaborative Filtering: This method identifies other consumers that have similar preferences, then recommends the products they would like. These "similar" users are commonly referred to as "social neighbors" [38]. The main disadvantage of the collaborative filtering method is the inadequacy in analyzing the product nature, since it only uses similarity between customer profile and neighbor customers.

Adomavicius, et al. [39] add one more category to the above classification:

Knowledge-based Filtering: This approach uses knowledge about users to recommend products that meet their requirements. Content based and collaborative approaches use ratings collected by users, so these approaches have cold start and data sparsity problems. The knowledge-based approach avoids these problems; however, it needs a knowledge base in advance.

Since each of the above methods has advantages and disadvantages, some studies combine these methods to provide more accurate recommendations [40].

Most recommendation systems are based on a single criterion such as the price of a product. However, a recent work shows that single-criterion value is limited, since the accuracy of the recommendation for a user usually depends on multiple criteria [41]. In addition, multi-criteria recommendation systems improve accuracy and suitability of the recommendations [39].

\subsubsection{Bounded Rationality, Satisficing Behavior, Heuristics, and Biases}

According to Herbert Simon's 'bounded rationality' theory, people may not possess the cognitive resources to make optimum decisions [42]. The 'bounded rationality' concept questions the notion of maximizing the utility. According to Simon, evaluation of all the relevant alternatives and their potential consequences is a challenge for optimization, due to the problem of limited resources and time [42]. Then, Simon propose the term 'satisficing' to describe a typical behavior of people, which is searching a solution until finding a satisfactory one that meets minimum requirements rather than an optimum solution.

Simon proposed that humans are limited in their rationality because of the following three factors. First, the rationality requires full understanding of the future consequences of a given action. Second, it is difficult for human to fully evaluate the future worth of their decisions, given those consequences of actions. Third, even though very few alternatives are known in actual decision-making processes, rationality requires that all alternative actions are known. According to Simon, these factors prevent humans in making optimum decisions [42].

Tversky and Kahneman developed their own perspective on bounded rationality [43]. According to Kahneman and Tversky, rather than an extensive algorithmic 
processing, evaluation under uncertainty often relies on three general-purpose heuristics. These simplifying heuristics are availability, representativeness, and anchoring and adjustment, which can lead to systematic and predictable errors or biases [43].

\subsection{Participatory Sensing}

Technological developments increase the capabilities of mobile devices in terms of sensing, storage, processing power and communication. Participatory sensing (PS) is a new paradigm that enables mobile devices to act as mobile sensing appliances. Campbell, et al. [44] published the first study on this paradigm in 2006, and dominated this paradigm as "People-Centric Urban Sensing" [44]. Burke, et al. [20] also published a study entitled as "Participatory Sensing" in 2006. Participatory sensing is also known as participatory urbanism [45], urbanet [46] and mobile sensing [47].

The participatory sensing approach uses mobile devices such as mobile phones and tablets for collection, analysis, and sharing of sensor data in order to form a body of knowledge, and actuation of people using this knowledge. Therefore, a participatory sensing framework generally consists of four successive phases: collect, analyze, share and actuate.

\subsubsection{The Collect Phase}

This phase includes collecting data from human-carried mobile sensors (such as sensors of mobile phones and wearable medical sensors), static sensors (such as sensors embedded to buildings), and human input (such as free-text input). Individuals and community groups are vital in the process of data collection.

User participation is the fundamental element of the participatory sensing systems because of the participatory nature of these systems. The main challenge of the participatory systems is motivating people to participate in collecting data, since these systems consume resources (e.g., battery and computation power) of mobile devices and participants' times. A person does not want to be a volunteer to submit their sensing data for a participatory sensing system unless he has a benefit from the system. Moreover, participants would tend to be free riders other than contributors [48]. Incentive mechanisms could help to address the participation challenge. For example, [49] propose a reward mechanism called "Reverse Auction Dynamic Price with Virtual Participation Credit (RADP-VPC)" incentive mechanism where users can sell their sensing data.

Collecting data without annoying the participant is another challenge. Application specific and automated data collection algorithms may address this problem. For example, [50] proposes a system for the automatic collection of fuel prices from mobile phone cameras of participants. 


\subsubsection{The Analyze Phase}

The analyze phase consists of analyzing the gathered data in the collect phase. In this phase, simple statistical techniques and complex methods such as machinelearning techniques are used to analyze sensed data, and transform collected data into meaningful information.

Data analysis is also an important and challenging part of a participatory sensing system. Detection of the physical activity type and the transportation mode is popular examples of data analysis in the participatory sensing systems. Activity classifiers and pattern recognition mechanisms [51], image classifiers [52], discrete Fourier transform [53], and machine learning [54] are used techniques for the data analysis in the participatory sensing systems.

\subsubsection{The Share Phase}

The share phase is required to disseminate the analyzed information. In this phase, the analyzed information is visualized on the mobile phone, back-end servers, and monitors in the vehicle, home or office. Web portals [55], social networks (e.g., Facebook, Twitter, Skype, Pidgin), and virtual world simulators [56] are some examples of the sharing mediums.

Preserving privacy of the participants is a key challenge for the share phase. For example, in a typical participatory sensing system, participants upload contextual information including geo-location and time. This situation leads violation of spatiotemporal privacy of participants, and discourages people from participating in the participatory sensing systems. Another challenge is to ensure the validity of the shared data. Authenticity, legality, accuracy and legibility of shared data are also important to the share phase.

\subsubsection{The Actuate Phase}

In this phase, an output of the participatory sensing system actuates an actuation agent (e.g., a person, a group of people or another system). Automated methods may match analyzed data and predefined patterns, and triggers actuation if they detect matches.

Ensuring the accuracy of actuation is the challenge for the actuate phase since inaccurate matches may cause serious problems. Developing algorithms to fuse the collected data, analyzed data, historical data, existing data (e.g., maps), and predefined patterns enable more accurate and complex actuation.

\subsection{Related Work}

In this section, the related work is classified into three groups. The first group consists of the studies related to the consumer decision process. The participatory 
sensing approach related studies are included in the second group. Finally, the third group includes the studies related to OCR.

\subsubsection{Consumer Decision Process Related Studies}

Rapid advances in information and communication technologies change the nature of consumer and business markets [57]. Consumers no longer act independently; they are increasingly connected with other consumers, brands and resources on the Internet via multiple digital channels, devices, and platforms $[57,58]$. The rapidly increasing connectivity also transforms shopping behavior and thinking ways of consumers [58].

Recent surveys show that developments in mobile device technologies are also changing shopping behavior $[59,60]$. For example, $88 \%$ of consumers create a shopping list [60]. $51 \%$ of these consumers use a handwritten shopping list, $30 \%$ of them create a shopping list on a mobile device saved as a note, $9 \%$ of consumers use a mobile app to create a shopping list, $8 \%$ of them email the shopping list to themselves, and $3 \%$ of them create a shopping list in a recipe app [60]. Consumers use information about sales, coupons, special offers, nutrition, nearby stores where the product is available, and reviews of the product before adding a product to their shopping lists.

In this section, mobile studies, which address specific tasks of the consumer decision process (CDP), such as price comparison [11, 61-63], product review [10, 64], reminder [65] applications, are presented. These studies in the literature are summarized in Table 2.2, sorted according to the corresponding stages they support within the consumer decision process. Related studies are described next, and further details of the correspondences with the stages are given in Chapter 4 . When the participatory sensing approach is applicable to these studies, correspondences with the phases of the participatory sensing are given in this section.

PromotionRank [66] is a study which uses items in the consumer's shopping list to generate a personalized ranking of grocery product promotions. After mapping items in the shopping list onto potentially relevant product categories, PromotionRank uses collaborative filtering to extend these categories. For each category, PromotionRank uses a statistical interest criterion to calculate the rank score for the category. Then, it ranks the available promotions using the newly computed rank scores. PromotionRank supports only the problem recognition stage of the consumer decision process by providing promotions.

Ma\$\$iv€ is a mobile grocery assistant designed around a shopping list paradigm [67], and primarily focuses on supporting consumers inside a grocery store. Accordingly, the authors conducted a survey study within a large supermarket to determine the functions of a mobile grocery aid. The authors report that customers care about features that facilitate budgeting and time usage (product price information, special offers and customer loyalty card offers, price comparison of 
similar products), and features that facilitate basic shopping tasks (shopping list, checkout, health information such as the total amount of fat in an item, product information such as ingredients and manufacturer), within a grocery aid running on mobile phones. Among these, they develop solutions for the following features: the natural language shopping list, product search, product recommendations and indoor positioning. Therefore, Ma\$\$iv€ facilitates the information search and evaluation of alternatives stages of the consumer decision process.

Table 2.2 Studies related to the individual stages of the CDP

\begin{tabular}{llll}
\hline Study & Type & Stage Supported & $\begin{array}{l}\text { Participatory } \\
\text { Sensing } \\
\text { Support }\end{array}$ \\
\hline $\begin{array}{l}\text { PromotionRank } \\
\text { [66] }\end{array}$ & $\begin{array}{l}\text { Personal } \\
\text { Promotions }\end{array}$ & Problem Recognition & No \\
\hline Ma\$\$iv€ [67] & Shopping List & $\begin{array}{l}\text { Information Search, } \\
\text { Evaluation of } \\
\text { Alternatives }\end{array}$ & No \\
\hline MobiShop [12] & Price Comparison & $\begin{array}{l}\text { Evaluation of } \\
\text { Alternatives }\end{array}$ & Yes \\
\hline LiveCompare [11] & Price Comparison & $\begin{array}{l}\text { Evaluation of } \\
\text { Alternatives }\end{array}$ & Yes \\
\hline My2cents [10] & Review & $\begin{array}{l}\text { Post-purchase } \\
\text { Evaluation }\end{array}$ & Yes \\
\hline Apriori [64] & Review & $\begin{array}{l}\text { Post-purchase } \\
\text { Evaluation }\end{array}$ & Yes \\
\hline
\end{tabular}

MobiShop [12] is a participatory sensing application that allows sharing of product prices among participants. MobiShop supports collecting, processing and sharing product prices from retail shops, for potential buyers. It uses receipt scanning as a product identification mechanism. Authors of MobiShop claim that retailers can use it as an indirect advertising medium. MobiShop is a client-server program; mobile phones act as clients, and a central web server with database acts as the server. According to the authors, it has two principal modes of operation: product price and user query.

In the sense phase, the participant takes a photo of the store receipt with the mobile phone camera. A store receipt includes the products and their corresponding prices. In the analyze phase, MobiShop extracts the pricing information from the image using native Symbian OS 9.2 OCR engine. In the share phase, extracted information is uploaded along with the Global Positioning System (GPS) coordinates and the time of purchase to the central server, and this server updates a database with received product prices at different stores. The authors assume that this database is 
interfaced to a Geographic Information System (GIS) map, which has the location of the stores. In the actuate phase, a participant can query for the prices of a particular product in his or her neighborhood, and makes a decision regarding the server reply.

MobiShop does not provide a product identification method besides receipt scanning. Therefore, product identification and data input are limited for MobiShop. In addition, it does not support elements of the consumer decision process except for the price comparison task within the evaluation of alternatives stage.

LiveCompare [11] is another participatory sensing system for grocery bargain hunting. It is a price comparison application that uses localization and barcodes in order to determine the location of the store and the products in the system, respectively. In the sense phase, participants capture an image of a product's price tag using a mobile phone camera. Generally, a barcode is included in the price tag, which uniquely identifies the product. In the share phase, the captured image is uploaded to a central database to satisfy future queries. Then, in the analyze phase, the central server analyzes the barcode, retrieves the Universal Products Code (UPC), finds the corresponding product, and sends previously recorded pricing information for the scanned product at other grocery stores. In the actuate phase, the participant uses the information sent by the server to make purchase decisions.

Since LiveCompare uses only barcodes to find the corresponding product, genericbrand products cannot be detected from their barcodes. Thus, these products cannot be compared across different grocery stores. In this case, OCR or human input may help to identify the product. Moreover, LiveCompare only detects the product barcode from the price tag; it does not extract other information such as the price of the product. Similar to MobiShop, LiveCompare only supports the price comparison task within the evaluation of alternatives stage.

My2cents [10] is a mobile application for product reviews. By using My2cents, consumers read comments about products as well as share their own comments and ratings with other users and within social networks. Thus, it combines social networking and mobile product reviews. It generates a product-based information stream in the form of micro-blogging such as using Twitter.

In the sense phase, participants scan a product's barcode and upload it. Then, My2cents web service returns basic product information and existing comments about the product in the analyze phase. In the share phase, participants submit their comments. When a participant submits a comment, this comment is added to the list of comments about the product. If the participant authenticates to Twitter and chooses to publish his or her comments on Twitter, the participant's comment is also shared on his or her Twitter stream.

My2cents facilitates the information search stage of the consumer decision process through sharing of product reviews among users, but it does not support other stages. 
APriori [64] is another product review system. In the sense phase, participants scan Radio-Frequency Identification (RFID) tags that are attached to products, and the APriori application retrieves the product identifier stored on the RFID tag. Then, the application queries a central server to retrieve existing ratings of the product. In the analyze phase, the central server receives the query and authenticates the user. After user authentication, the query is forwarded to the rating database. Then, the product rating information is sent to participants and they can take a purchase decision.

APriori partly support the post-purchase evaluation stage by providing product review functionality, but they do not target the other stages of the consumer decision process.

As briefly mentioned in this chapter and shown in Table 2.2, there are studies supporting individual stages of the consumer decision process, in the form of specific activities of consumers, such as comparing prices and searching for reviews of products. On the other hand, MobileCDP offers a holistic and modular approach to address the challenges of consumers in their activities by using the wellestablished consumer decision process as its foundation. Accordingly, the design of MobileCDP also allows integration of algorithmic solutions offered by such aforementioned related studies into the framework.

\subsubsection{Participatory Sensing Related Studies}

In this section, example participatory sensing studies are given for the main application areas of the participatory sensing approach, which are environmental monitoring, traffic and transportation, social networking, health, and well-being.

\section{Environmental monitoring studies:}

Personal Environmental Impact Report (PEIR) [68] is a participatory sensing project to evaluate participants' impact on the environment through using geo-temporal data gathered with mobile phones. PEIR allows participants to perceive the effects of their behavior on themselves and the environment. NoiseTube [69] is a project to turn GPS enabled mobile phones into mobile noise sensing devices to measure participants' personal exposure to noise. VUPoints [70] is a video-recording and collaborative sensing system that uses mobile phones to sense their environment. It catches events that qualify for recording in a collaborative manner. SoundSense [71] is a scalable sound sensing framework to model sound events on smartphones. The SoundSense project presents an event classification system for audio. This system classifies general audio types (e.g., voice, music) and detects specific audio events by using the sensors of mobile phones. NoiseSpy [72] is a sound-sensing system that uses mobile phones to monitor environmental noise. Ear-Phone [73] is an endto-end noise pollution mapping system based on PS like NoiseTube [69]. Sensing atmosphere [45] and Common Sense [74] are other examples or environmental participatory sensing applications. 


\section{Traffic and transportation studies:}

Pothole Patrol [54] is a participatory sensing system that opportunistically collects the vibration data as well as the location data gathered from GPS sensors embedded in vehicles, and processes the data to assess road surface conditions. Nericell [75] is a similar system but more complex than Pothole Patrol. Traffic conditions are also monitored in Nericell, using mobile phones' sensors (e.g., accelerometer, microphone, GSM radio, and GPS). Vtrack [76], GreenGPS [77], ParkNet [78], and Fueoogle [79] are other traffic and transportation type participatory sensing applications.

\section{Social networking studies:}

CenceMe $[53,80]$ is a participatory sensing system for personal use. Users of social community networks such as Facebook, MySpace and Twitter, can share their sensing presence with their friends using CenceMe application. CenceMe application automatically gathers human presence information based on sensors of mobile phones in order to share users' context. Second Life [81] is a virtual world simulator, which allows users to interact with each other through mobile avatars. Second Life's 41 million users can socialize and participate activities in the 3D virtual world using voice and text chat. Other examples of social networking type participatory sensing applications are MoVi [82], Social Fusion [83], and Virtual Compass [84].

\section{Health and well-being studies:}

BikeNet $[55,85]$ is developed for collecting and sharing data measuring various aspects of the cycling experience. Biketastic [51] is a similar project to the BikeNet. It supports bikers to share their routes with other bikers, and allows them to improve their routes for decreasing traffic accident probability, and increasing air and roadsurface quality. Dietsense [52] uses automatic image processing techniques and manual image review to automatically collect multimedia documentation of dietary choices. This documentation is stored in secure accounts on web servers for selfreview of users and dietary specialists. SPA [86] is a participatory sensing system used for health self-management. Its main functions are gathering real-time environmental and biomedical data from participants using mobile phones' sensors, finding relationships between the environmental and biomedical parameters, and automatically triggering alarms and on-line surveys. UbiFit Garden [87] and SHIELD [88] are other participatory sensing applications for health and-well-being.

\section{Participatory sensing platforms:}

In the literature, there are also platforms that facilitate development of participatory sensing applications. AnonySense $[89,90]$ is a privacy-aware general-purpose opportunistic sensing architecture for leveraging users' mobile devices for measuring context in a target region. It presents a framework that supports 
applications to submit sensing tasks to be distributed across collaborating mobile devices and provides nodes to receive tasks anonymously. Campaignr [91] is a software platform that allows people to participate in data gathering campaigns with their mobile phones. Campaignr does not offer a solution to publish, share or manage collected data. NORS (Nokia Remote Sensing) is an open source platform that facilitates PS applications, which is developed at Nokia Research Center [92]. NORS enables mobile phones to act as sensor gateways to perform both local and remote sensing. PRISM (Platform for Remote Sensing using Smartphones) [93] is a platform, which tries to simplify developing and deploying applications in addition to balance three interconnected goals: generality, security, and scalability.

\subsubsection{OCR Related Studies}

Recent developments related to mobile devices have led to an increase in their digital imaging capabilities as well as the emergence of new approaches in solving problems such as automated data collection by using Optical Character Recognition (OCR) for automatic extraction of various information from images captured by mobile phone cameras. The OCR method is also used for data collection in participatory sensing studies. In our research, we used OCR to collect data for the components of the framework.

The amount of published literature on information extraction from store receipts is rather limited. The following studies are related to automated data collection within the participatory sensing approach by using OCR for automatic extraction of various information from images captured by mobile phone cameras.

OCRdroid [94] is a framework for developing OCR-based applications on mobile phones, which provides image processing based solutions for OCR problems in mobile phone captured images. Bad orientation, text misalignment, text skew and insufficient lighting are some of these OCR problems. OCRdroid uses an image based auto-rotation algorithm to detect and correct text skew, and image binarization methods for lighting problems. It also utilizes embedded sensors of the mobile phone to detect text misalignment and orientation problems, and guides users to help them align the text properly. The authors tested various open source OCR engines, and they state that the Tesseract OCR engine [95] gives the best results. According to the authors, the correct character recognition rate of OCRdroid changes between $66 \%$ and $97 \%$ under various conditions.

MobiShop[12], also mentioned in the consumer decision process related studies (Chapter 2.3.1), is also an OCR related study that enables the photo capture of store receipts, and extraction of product names and corresponding prices from such photos. The authors state that Mobishop has $60 \%$ correct word recognition rate. Because of the high probability of the recognition errors, they also added a feature that enables editing of the extracted text by the user in order to fix the recognition errors. 
Both Mobishop and OCRdroid use mobile phones and open source OCR engines. However, they do not provide sufficient details regarding their implementations. In addition to these studies, there are also other studies using commercial OCR engines and specialized hardware. For example, Receiptlog [96] determines shopping habits of costumers to predict their future behavior by using OCR on store receipts to collect information about past purchases.

In this research, we also propose a novel approach for improved OCR, especially in the case of low quality video captured by a handheld mobile phone. Since our main goal is to improve the OCR accuracy, the proposed method combines OCR outputs from multiple frames in order to extract and use the most reliable information present in the individual frames.

Super resolution (SR) is an approach that uses signal processing techniques for obtaining a high resolution (HR) image from a set of low-resolution (LR) observations [97]. The source of these observations can be multiple sensors capturing a single scene, or a single sensor capturing the scene over a period of a time [98], such as a mobile phone captured video.

Super resolution algorithms use various techniques for reconstructing a high resolution image. One of them is a frequency domain technique, which registers low resolution images based on low-frequency, aliasing-free parts [99]. L1 norm minimization [100], Tikhonov regularization [101], the total variation (TV) method [102], and the robust super resolution (Zomet) method [103] are other well-known methods for reconstruction of a high resolution image from low resolution images. In [100], the authors show that the performance of L1 norm minimization is superior to the methods in [101-103]. Accordingly, the L1 norm method is used as the super resolution method in our experiments for comparison purposes.

There are two major challenges associated with super resolution. The first one is the image registration/motion estimation, which is precisely determining the difference between low resolution images; and the second one is the image reconstruction, which is reconstructing a high resolution image by combining information obtained from the registered images [99]. Motion estimation and reconstruction errors cause some degradation of the resulting high resolution image, and a reduction in the accurate recognition rate of the OCR operation applied to the image.

\subsection{Chapter Summary}

This chapter reviewed key literature in the fields of the consumer decision process, mobile information systems as well as participatory sensing, and presents the relevant knowledge from these fields for this research.

The consumer decision process is an important area in the consumer domain, and includes the problem recognition, information search, evaluation of alternatives, purchase decision, and post-purchase evaluation stages $[1,4,18]$. Similarly, the 
participatory sensing paradigm is an emerging topic in the mobile technology domain. A participatory sensing system generally consists of four successive phases: collect, analyze, share and actuate. Environmental monitoring, traffic and transportation, social networking, health, and well-being are example application areas of the participatory sensing approach.

In the literature, there are solutions in the form of mobile information systems. Although some of these solutions also support the participatory sensing approach, they provide limited support to address challenges of consumers, and assist individual stages of the consumer decision process, such as information search and evaluation and alternatives. This research addresses this gap to help consumers in their activities by providing a holistic approach for supporting all stages of the consumer decision process. 


\section{CHAPTER 3}

\section{RESEARCH METHODOLOGY}

As an applied research discipline, Information Systems (IS) apply theory from other disciplines, such as the computer science and the social sciences, to solve problems at the intersection of information technology (IT) [26]. For example, our research applies theory from a social science domain (consumer behavior) to solve problems of consumers using the mobile technologies as the intersection of IT. Following a suitable research methodology empowers the research for contributing to the body of knowledge in a certain discipline [26].

This chapter presents the research methodology based on literature, and shows rigor and relevance of our research with this research methodology by explaining and justifying the choice of the applied research methodology.

\subsection{Design Science Research}

In recent years, several researchers show the validity and value of Design Science Research (DSR) as a research paradigm that specialized for IS discipline [25-27]. In addition, March and Storey [104] state that "design science research is increasingly recognized as an equal companion to behavioral science research in the information systems field", in the introduction of the MISQ special edition on DSR. Accordingly, the DSR paradigm is chosen as the research methodology of this study.

\subsection{Research Process}

Peffers, et al. [26] suggest a DSR methodology, which is consistent with prior literature, and provides Design Science Research Methodology (DSRM) Process Model to present the DSR. The authors claim that this process model provides support for researchers, and this is "a good way" to do DSR [26]. In this section, we justify that the DSRM process model of Peffers, et al. [26] is an appropriate choice for our study, and validate our research methodology against DSR guidelines of Hevner, et al. [25]. 


\subsubsection{The Research Entry Point}

As shown in Figure 3.1, DSRM process model consists of six activities, which cover the entire research from the start (motivation) to the end (communication). This model designed as successive activities, however, according to Peffers, et al. [26], the Research Entry Point varies depending on the actual project. There are four possible research entry points: the problem-centered initiation (e.g., finding a research gap in the literature), objective-centered solution (e.g., industrial projects), design and development centered initiation, and client/context initiated research (e.g., real world consulting projects).

For this research, the entry point is "the problem centered initiation"; we identify a problem in the literature and investigate this problem in the research process. As shown in the figure, this entry point implies that the research process will be followed in sequential order, starting with the first activity, "identify problem and motivate".

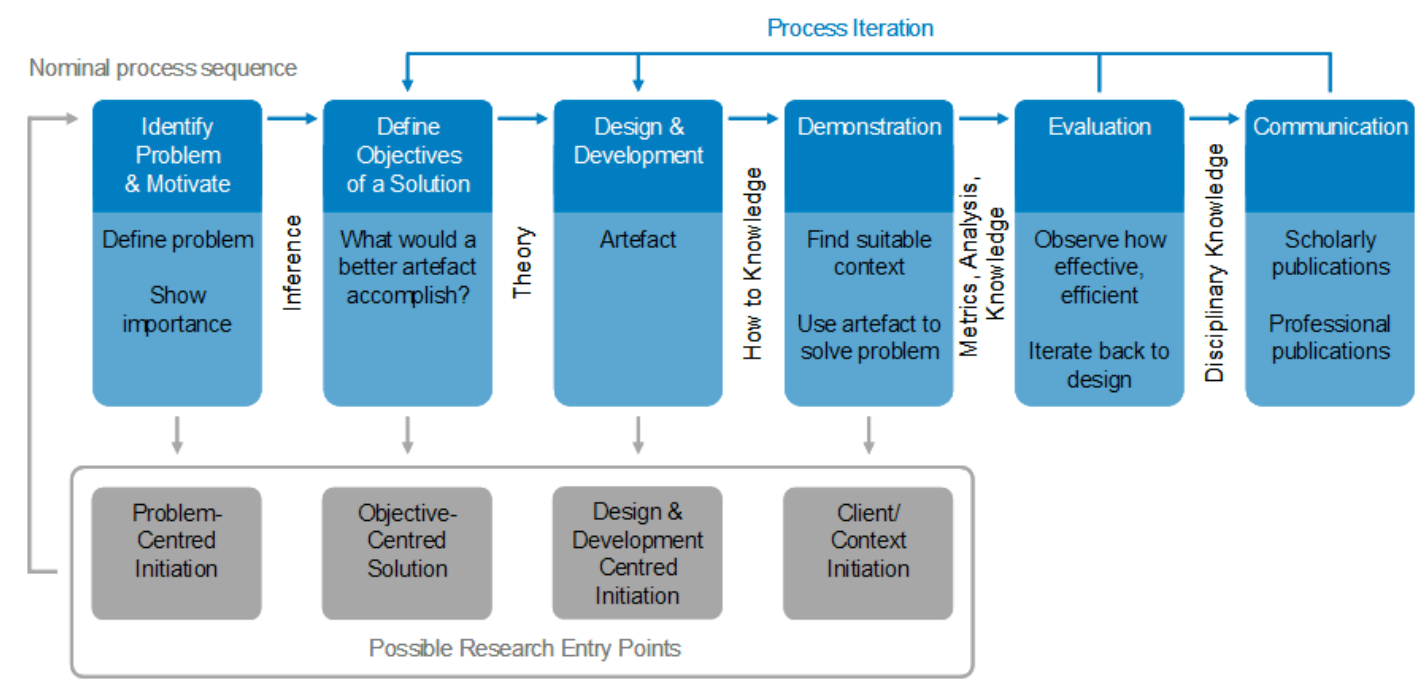

Figure 3.1 Design science research methodology process model (adapted from Peffers, et al. [26])

\subsubsection{Activity 1: Identify Problem and Motivate}

In the DSRM process model, the first activity is the definition of the specific research problem, and justification of the "value of a solution" [26].

The focus of this research is how the challenges of consumers in their activities can be addressed. It aims to show that a mobile information system framework that supports each stage of the consumer decision process can assist consumers in their 
activities, and the participatory sensing approach can empower such a framework by providing collective data.

\subsubsection{Activity 2: Define Objectives of a Solution}

The second activity of the DSRM process model includes defining the objectives of the proposed solution, while the first activity includes the general identification of the problem (the gap). These objectives can be quantitative, for example, "a desirable solution would be better than current ones", or qualitative, such as "a description of how a new artifact is expected to support solutions to problems not hitherto addressed" [26].

According to these definitions, our research has qualitative objectives, and Chapter 4 describes the advantages of the MobileCDP framework and how it can support consumers in their activities, Chapter 6 and Chapter 7 justifies applicability of the framework and advantages for consumers.

\subsubsection{Activity 3: Design and Development}

This activity is defined as "create the artifact" [26]. This activity includes determining the desired functionality of the artifact as well as its architecture, then creating the actual artifact. According to the authors, these artifacts are potentially constructs, models, methods, and instantiations [26].

In this research, the artifact (the MobileCDP framework) is a model, which is designed and developed based on the identified objectives.

\subsubsection{Activity 4: Demonstration}

The fourth activity includes the demonstration of the proposed artifact. This demonstration can be experimentation, simulation, case study, proof, prototype, or other appropriate activities that show the use of the proposed artifact in solving one or more instances of the problem. Effective knowledge is required to how to use the artifact to solve the problem.

In this research, the prototype implementation of MobileCDP is the demonstration of the framework. The purpose of the developed prototype is to show the feasibility of the artifact.

\subsubsection{Activity 5: Evaluation}

This activity includes observing and measuring how well the artifact supports a solution to the problem, and involves a comparison between the objectives of a solution and actual observed results that are obtained by using the artifact in the demonstration. According to Hevner, et al. [25], the evaluation of the artifact is a crucial part of a DSR process. They classified suitable evaluation methods in five categories as seen in Table 3.1. 
Table 3.1 Design science research evaluation methods (adapted from [25])

\begin{tabular}{ll}
\hline Category & Evaluation Method \\
\hline Observational & Case Study: Study artifact in depth in business environment \\
& Field Study: Monitor use of artifact in multiple projects \\
\hline & Static Analysis: Examine structure of artifact for static qualities \\
& (e.g., complexity) \\
& Architecture Analysis: Study fit of artifact into technical IS \\
& architecture \\
& Optimization: Demonstrate inherent optimal properties of artifact \\
& or provide optimality bounds on artifact behavior \\
Dynalytical & (e.g., performance) \\
Controlled Experiment: Study artifact in controlled environment \\
for qualities (e.g., usability) \\
Simulation - Execute artifact with artificial data \\
Experimental & $\begin{array}{l}\text { Functional (Black Box) Testing: Execute artifact interfaces to } \\
\text { discover failures and identify defects } \\
\text { Structural (White Box) Testing: Perform coverage testing of some } \\
\text { metric (e.g., execution paths) in the artifact implementation }\end{array}$ \\
\hline Informed Argument: Use information from the knowledge base \\
(e.g., relevant research) to build a convincing argument for the \\
artifact's utility \\
Scenarios: Construct detailed scenarios around the artifact to \\
demonstrate its utility
\end{tabular}


Considering the categorization of the evaluation methods, the main method to evaluate the proposed MobileCDP artifact is experimental in form of a controlled experiment, which is studying the artifact in a controlled environment for qualities, such as usability.

\subsubsection{Activity 6: Communication of Research}

Communication of a design science research is one of the most crucial activities in research [25-27]. Scholarly research publications and the $\mathrm{PhD}$ thesis are examples of such a communication.

This $\mathrm{PhD}$ thesis is a detailed and comprehensive piece of communication of this research. Gregor and Hevner [27] provide a publication schema to appropriately communicate design science research projects as shown in Table 3.2. A new column added to schema to indicate corresponding chapters of this thesis for each section of the publication schema.

In addition, published conference proceedings and submitted journal papers provide communication of this research.

\subsection{Validation of the Research Methodology}

In this section, the research methodology of this research is evaluated against seven guidelines suggested by Hevner, et al. [25]. According to the authors, their purpose to provide these guidelines is assisting researchers, reviewers, editors, and readers in understanding the requirements for effective design science research [25]. Table 3.3 summarizes the seven guidelines. Next, we validate the research methodology of our research against each guideline.

\subsubsection{Guideline 1: Design as an Artifact}

Guideline 1 requires that the result of the design-science research in Information Systems is a purposeful artifact for a specified problem domain. This guideline is also stated that the artifact must enable its implementation and application in an appropriate domain.

Hevner, et al. [25] also define the artifact as follows:

"Furthermore, artifacts constructed in design-science research are rarely fullgrown information systems that are used in practice. Instead, artifacts are innovations that define the ideas, practices, technical capabilities, and products through which the analysis, design, implementation, and use of information systems can be effectively and efficiently accomplished."

In this research, the MobileCDP framework is the artifact, which is built on examining literature and addressing the identified gap. 
Table 3.2 DSR publication schema (adapted from Gregor and Hevner [27])

\begin{tabular}{ll}
\hline Section & Contents \\
\hline $\begin{array}{l}\text { Problem definition, problem significance/motivation, } \\
\text { introduction to key concepts, research questions/objectives, } \\
\text { scope of study, overview of methods and findings, theoretical } \\
\text { and practical significance, structure of remainder of paper. }\end{array}$
\end{tabular}

Thesis

Chapters

For DSR, the contents are similar, but the problem definition

Chapter 1

and research objectives should specify the goals that are required of the artifact to be developed.

Prior work that is relevant to the study, including theories, empirical research studies and findings/reports from practice.

2. Literature Review

For DSR work, the prior literature surveyed should include any

Chapter 2 prior design theory/knowledge relating to the class of problems to be addressed, including artifacts that have already been developed to solve similar problems.

The research approach that was employed.

3. Method

For DSR work, the specific DSR approach adopted should be

Chapter 3 explained with reference to existing authorities.

A concise description of the artifact at the appropriate level of abstraction to make a new contribution to the knowledge base.

4. Artifact Description

This section (or sections) should occupy the major part of the paper. The format is likely to be variable but should include at least the description of the designed artifact and, perhaps, the

Chapter 4

Chapter 5

Chapter 6 design search process.

\section{Evidence that the artifact is useful.}

5. Evaluation

The artifact is evaluated to demonstrate its worth with evidence

Chapter 7 addressing criteria such as validity, utility, quality, and efficacy.

Interpretation of the results: what the results mean and how they relate back to the objectives stated in the Introduction section. Can include: summary of what was learned, comparison with prior work, limitations, theoretical significance,

6. Discussion practical significance, and areas requiring further work.

Chapter 8

Research contributions are highlighted and the broad implications of the paper's results to research and practice are discussed.

Concluding paragraphs that restate the important findings of the work.

7. Conclusions

Restates the main ideas in the contribution and why they are important. 
Table 3.3 Design science research guidelines (adapted from Hevner, et al. [25])

\begin{tabular}{|c|c|}
\hline Guideline & Description \\
\hline $\begin{array}{l}\text { Guideline 1: } \\
\text { Design as an Artifact }\end{array}$ & $\begin{array}{l}\text { Design-science research must produce a viable } \\
\text { artifact in the form of a construct, a model, a } \\
\text { method, or an instantiation. }\end{array}$ \\
\hline $\begin{array}{l}\text { Guideline 2: } \\
\text { Problem Relevance }\end{array}$ & $\begin{array}{l}\text { The objective of design-science research is to } \\
\text { develop technology-based solutions to important } \\
\text { and relevant business problems. }\end{array}$ \\
\hline $\begin{array}{l}\text { Guideline 3: } \\
\text { Design Evaluation }\end{array}$ & $\begin{array}{l}\text { The utility, quality, and efficacy of a design artifact } \\
\text { must be rigorously demonstrated via well-executed } \\
\text { evaluation methods. }\end{array}$ \\
\hline $\begin{array}{l}\text { Guideline 4: } \\
\text { Research Contributions }\end{array}$ & $\begin{array}{l}\text { Effective design-science research must provide } \\
\text { clear and verifiable contributions in the areas of the } \\
\text { design artifact, design foundations, and/or design } \\
\text { methodologies. }\end{array}$ \\
\hline $\begin{array}{l}\text { Guideline 5: } \\
\text { Research Rigor }\end{array}$ & $\begin{array}{l}\text { Design-science research relies upon the application } \\
\text { of rigorous methods in both the construction and } \\
\text { evaluation of the design artifact. }\end{array}$ \\
\hline $\begin{array}{l}\text { Guideline 6: } \\
\text { Design as a Search Process }\end{array}$ & $\begin{array}{l}\text { The search for an effective artifact requires utilizing } \\
\text { available means to reach desired ends while } \\
\text { satisfying laws in the problem environment. }\end{array}$ \\
\hline $\begin{array}{l}\text { Guideline 7: } \\
\text { Communication of Research }\end{array}$ & $\begin{array}{l}\text { Design-science research must be presented } \\
\text { effectively both to technology-oriented as well as } \\
\text { management-oriented audiences. }\end{array}$ \\
\hline
\end{tabular}

\subsubsection{Guideline 2: Problem Relevance}

The "problem relevance" guideline states that the aim of the design science research in Information Systems must be develop solutions to relevant business problems. The problem domain of the MobileCDP framework is the consumer behavior domain, and its aim is to solve problems faced by consumers in the consumer decision process.

\subsubsection{Guideline 3: Design Evaluation}

Guideline 3 requires an evaluation of the artifact regarding its utility, efficacy, and quality by using well-executed evaluation methods [25]. As mentioned in Chapter 3.2.6, the controlled experiment method is chosen among the suggested methods by Hevner, et al. [25], to evaluate the MobileCDP framework. This evaluation method studies the artifact in a controlled environment for qualities such as usability. Details of the used evaluation methods are given in Chapter 7.1.1. 


\subsubsection{Guideline 4: Research Contributions}

Hevner, et al. [25] identify three different types of contributions as an output of a design science research: the design artifact, foundations, and methodologies.

The first type of research contribution is the design artifact. Hevner, et al. [25] state, "Most often, the contribution of design science research is the artifact itself. The artifact may extend the knowledge base, or apply existing knowledge in new and innovative ways. System development methodologies, design tools, and prototype systems (e.g., GDSS, expert systems) are examples of such artifacts". The second type is "foundations", which extend and improve the existing foundations in the design-science knowledge base. "Methodologies", as the third type of the research contribution", includes the creative development and use of evaluation methods, and new evaluation metrics.

Considering above descriptions of the three research types, the main contribution of this research is the design artifact itself (the MobileCDP framework) by applying existing knowledge in new and innovative ways, and extending the knowledge base.

\subsubsection{Guideline 5: Research Rigor}

According to Hevner, et al. [25], " design science research requires the application of rigorous methods in both the construction and evaluation of the designed artifact". The authors state that designed artifacts are often components of a human-machine problem-solving system, and knowledge of behavioral theories are necessary to construct such artifacts [25].

These definitions are exactly compatible with this research. We construct the artifact of this research (MobileCDP) using the existing knowledge of the consumer behavior theory. Design and construction of the MobileCDP are described in Chapter 4.

\subsubsection{Guideline 6: Design as a Search Process}

Design science research is an iterative process that aims to find an effective solution for the specified problems by using the knowledge base. This approach is named as "search process" by [25].

In this research, we reviewed the literature to identify components of the MobileCDP framework, and a selection of literature is given in Chapter 2. Several iterations were carried out until to find an effective solution, and new components were added or existing components have improved in each of the iterations. 


\subsubsection{Guideline 7: Communication of Research}

According to Hevner, et al. [25], DSR must be presented effectively. This thesis and published as well as submitted conference and journal papers consist of communication of this research with the academic audience.

\subsection{Chapter Summary}

This chapter develops and justifies a research methodology for our research. We choose the design science research paradigm as the research methodology, which is an appropriate approach to investigate problems in the Information Systems domain [25]. Supplemented with the DSRM research process model of Peffers, et al. [26], which is described in Chapter 3.2, the design science research paradigm outlines the research activities, ensures a rigorous research process, and presents a complete research methodology [25, 26]. 


\section{CHAPTER 4}

\section{THE MobileCDP FRAMEWORK}

In this research, the MobileCDP framework is proposed, which is based on the consumer decision process with the participatory sensing approach, and therefore incorporates the problem recognition, information search, evaluation of alternatives, purchase decision, and post-purchase evaluation stages $[1,4,18]$, as shown in the top portion of Figure 4.1. It specifically aims at addressing the challenges faced by consumers in their activities, and facilitates the development of mobile information systems for the whole consumer decision process by providing relevant components as demonstrated in Figure 4.1.

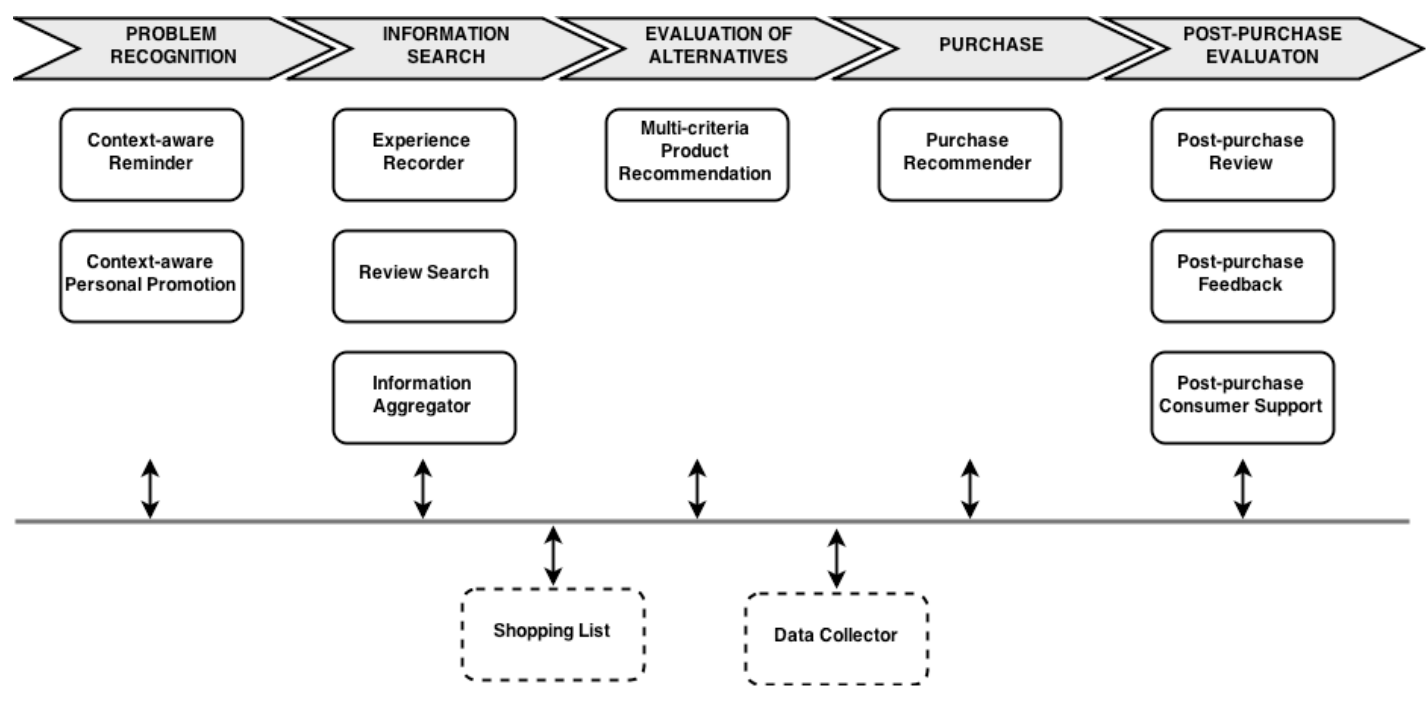

Figure 4.1 Components of the MobileCDP framework

MobileCDP includes twelve components that support the consumer decision process, as shown in Figure 4.1. On the basis of functionality, these components are classified into two main types: facilitating and unifying components. A facilitating component targets an individual stage of the consumer decision process to help consumers complete the stage effectively and efficiently. A unifying component is 
responsible for integration and coordination among the facilitating components. Each component is proposed based on the corresponding identified studies in the literature, as listed in Table 4.1. Due to the modular approach, the design allows incorporation of existing work in the literature into the framework, as well as extensions of the framework by adding new components.

The ten facilitating components are described next, including the challenges of the stage they correspond to, followed by descriptions of the two unifying components. Figure 4.2 illustrates these components as well as data sources, and the data exchange among them.

\subsection{Components for the Problem Recognition Stage}

In the problem recognition stage, consumers identify a need, and the type of product that satisfies this need. Typically, consumers record the needed product to their memory after recognizing the need, but it is possible that they forget to purchase the needed products [4]. Internal or external stimuli are required to trigger the problem recognition, and accordingly, two components are included to help consumers in this stage by creating these stimuli.

Table 4.1 The proposed components and justifying studies in the literature

\begin{tabular}{lll}
\hline Component & Assisted Stage & $\begin{array}{l}\text { Justifying } \\
\text { Research }\end{array}$ \\
\hline Context-aware Reminder & Problem Recognition & {$[105,106]$} \\
\hline Context-aware Personal Promotion & Problem Recognition & {$[4,107,108]$} \\
\hline Experience Recorder & Information Search & {$[4]$} \\
\hline Review Search & Information Search & {$[109,110]$} \\
\hline Information Aggregator & Information Search & {$[111-115]$} \\
\hline Multi-criteria Product Recommendation & Evaluation of Alternatives & {$[39,116,117]$} \\
\hline Purchase Recommender & Purchase & {$[34,105]$} \\
\hline Post-purchase review & Post-purchase Evaluation & {$[10,64,118]$} \\
\hline Post-purchase feedback & Post-purchase Evaluation & {$[119,120]$} \\
\hline Post-purchase consumer support & Post-purchase Evaluation & {$[105]$} \\
\hline
\end{tabular}




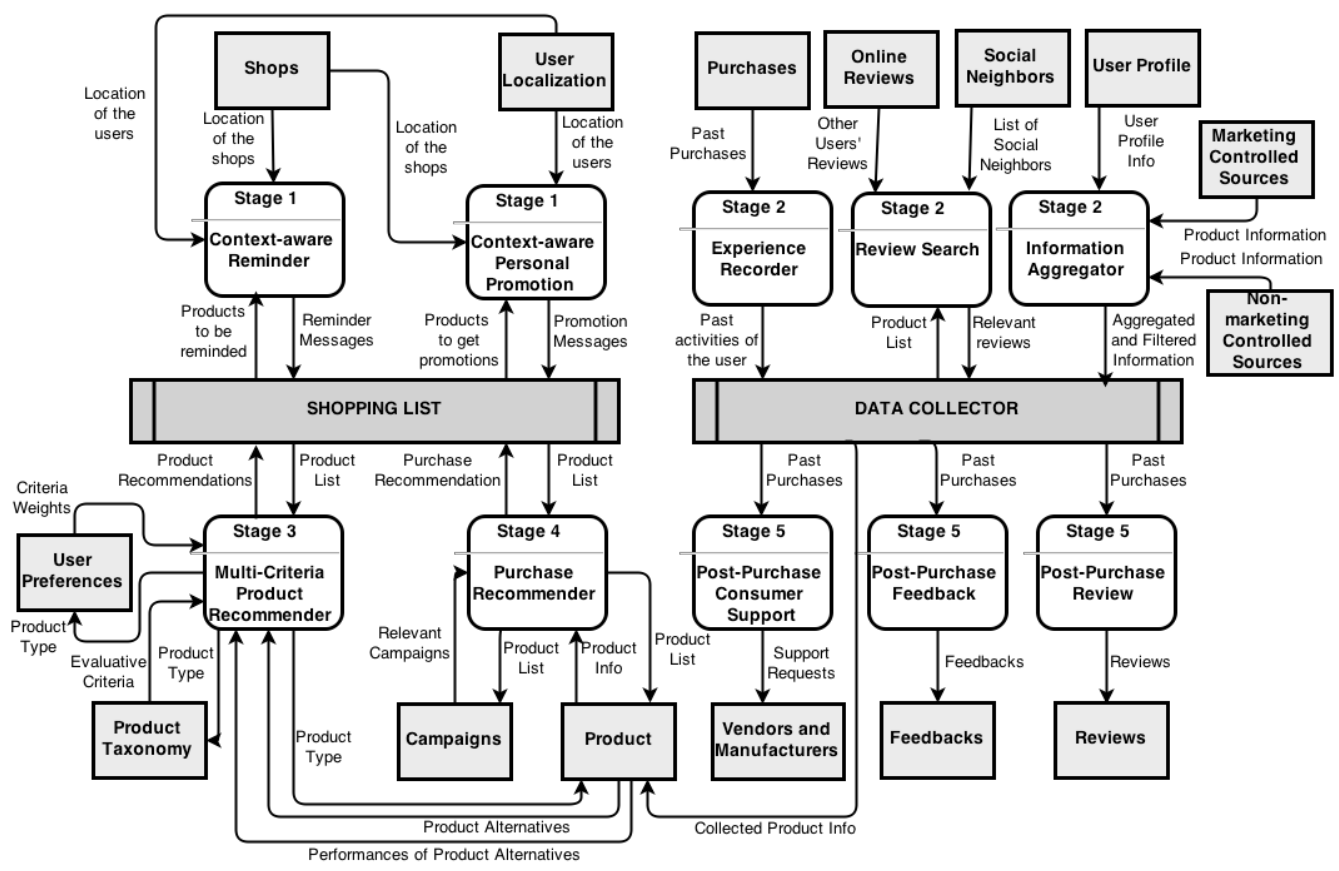

Figure 4.2 Data exchange among the components of the MobileCDP framework

\subsubsection{Context-aware Reminder Component}

Reminders are mentioned as a stimulator mechanism for the problem recognition stage [105]. They also serve as a complement to short-term memory [106]. Similarly, mobile studies such as CybreMinder [121] support users in sending and receiving reminders that can be associated with context such as time and place.

The context-aware reminder component of MobileCDP generates the required stimuli to help the consumer in the problem recognition stage. Reminder messages are delivered through the mobile phone of the consumer to invoke a need. Briefly, this component periodically checks the location of the consumer, the products to be reminded about, and stores that carry these items. If the current location of the consumer matches the location of a shop, which carries a product to be reminded about, the component shows a reminder message to the consumer.

\subsubsection{Context-aware Personal Promotion Component}

According to "grand models" mentioned in Chapter 1, advertising efforts are examples of external stimuli [13-15]. Mobile advertising text messages may initiate the consumer decision process [107]. In the problem recognition stage, it is possible to stimulate user interest for certain items through advertising or special offers [119]. Context-aware and personalized advertising, which aggregate user profile and location information, offers smart mobile advertisements [108]. 
The context-aware personal promotion component provides marketer initiated promotions and coupons to the consumer by using the items in the consumer's shopping list, as suggested by PromotionRank [66]. Briefly, consumers choose products in their shopping lists if they would like to receive such messages. Then, this component periodically looks for a match between location and time based offers of marketers, the location of the consumer and chosen products. If the component finds a match, it shows the corresponding offer to the consumer. The important point is that marketers are not allowed to directly send advertising messages to the consumer.

\subsection{Components for the Information Search Stage}

After an awareness of need has been built about the product needed, consumers pass to the second stage of the consumer decision process, information search. Consumers take action to obtain knowledge in this stage, and they perform internal or external information search operations to retrieve related information $[1,4,18]$. In internal search, consumers search their memory for information about products based on experience regarding the product, information obtained from past marketing advertisements, and information collected from the word of mouth (WOM) recommendations. However, consumers have cognitive limitations to recall information from memory [2]. In external search, consumers search for additional information with a focus on personal sources such as friends, public sources such as social networks, and marketer-dominated sources such as company web sites.

Three components described next facilitate the information search stage by collecting the required information, reducing information overload, and presenting related and filtered information to consumers. These components also address the challenge of cognitive limitation of consumers to recall information from memory, by presenting previously recorded information.

\subsubsection{Experience Recorder Component}

In internal information search, consumers recall relevant information stored in their long-term memory, such as previous experience with a product [4]. Therefore, in order to help with internal information search, previous activities of the consumer are recorded, and relevant recorded information is recalled when the consumer needs it. These activities include past purchases, previous product comparisons, and experiences with products such as reviews and feedback.

The experience recorder component of MobileCDP facilitates the internal search by recording the history of all activities such as purchases, product comparisons and reviews of the consumer, and recalling these records when the consumer needs them. 


\subsubsection{Review Search Component}

Recent advances in the web and mobile technologies have caused a proliferation of online consumer reviews, which contain product evaluations and opinions submitted by consumers. Studies in the literature state that online consumer reviews have become an important source of information that facilitates the consumer decision process [109, 110].

The review search component collects product reviews from online sources such as social networking sites, blogs, discussion forums, online retailers' sites, and online review sites. It also determines social neighbors by aggregating social network contacts such as Facebook and Twitter, as well as phone contacts. In addition, by analyzing past purchases of a consumer, it identifies other consumers purchasing similar products. Determining social neighbors also helps provide recommendations about new products. For example, Lawrence, et al. [122] designed a recommender system to suggest new products to supermarket consumers using previously purchased products of the consumer and matching a consumer with a group of consumers with similar spending histories.

\subsubsection{Information Aggregator Component}

Utilizing the Internet to find more information regarding considered purchases can increase consumers' sense of uncertainty [123]. According to the theory of information overload, the consumer may spend more effort and time to process information and may make lower quality decisions in case of information load increasing beyond a threshold [124]. Research shows that online information overload results in less satisfied, less confident, and more confused consumers [125]. However, even though decision-makers who believe to be underloaded make higher quality decisions, they feel less satisfied and confident than decision-makers who feel overloaded [126]. Different approaches have been proposed to address the information overload problem such as personalization, information filtering, and recommendation [127-129]. Likewise, information aggregator systems facilitate handling of information overload, by aggregating heterogeneous information sources, and presenting the results to the user in a personalized manner [111].

The information aggregator component in MobileCDP is intended to make automated information retrieval from non-marketing and marketing controlled sources, and also to present the obtained information to the consumer in a personalized manner. Non-marketing controlled sources include consumer reports, social networks, and other product information sources that are not associated with advertising, whereas marketing controlled sources include advertisements, promotions and other product information generated by marketers. The information aggregator component classifies marketing and non-marketing controlled sources, and presents filtered information according to its source. 
Sun [113] proposes the IRE (Information Requirement Elicitation) framework to facilitate information search for mobile users by providing a context-aware, interactive and personalized information search service. The IRE framework can be used within the information aggregator component of MobileCDP.

By providing a matching between the user's profile and product information, this component filters product alternatives that are incompatible with the user's profile, similar to the iGrocer study [112] that makes a compatibility check between the user's health profile and nutrition content of the food product.

\subsection{Components for the Evaluation of Alternatives Stage}

In this stage, alternatives are evaluated and selected to satisfy the needs. However, consumers have cognitive limitations while selecting and evaluating alternatives. Consumer behavior is characterized by a trade-off between the cost of evaluating more alternative products and information search. When more alternatives are taken into account, a better decision is possible [130]. Westerman, et al. [114] show that the quality of decisions is better when participants have access to a decision support system. A recent study shows that simple decision aids provided by many online retailers, such as the ability to sort on a particular attribute or eliminate undesired alternatives, decreases decision quality, especially when choice conflict is high [115]. The same study shows that using multiple simple decision aids such as combining elimination and sorting aids improves decision quality.

A number of studies show that recommendation aids increase consumers' decision quality. For example, Haubl and Trifts report that recommendation agents increase consumers' confidence and satisfaction with their purchase decisions [116]. Pereira [117] states that providing well-designed decision aids to consumers may significantly increase consumers' confidence, satisfaction, and decision quality. Most decision aids are based on a single criterion, such as the overall rating of a product by the consumer. However, recent work shows that a single-criterion value is limited since the accuracy of the recommendation may depend on more than one criterion, and multi-criteria recommendation systems improve accuracy and suitability of the recommendations [39]. Accordingly, a multi-criteria product recommendation component is included in this stage.

\subsubsection{Multi-criteria Product Recommendation Component}

The multi-criteria product recommendation component of MobileCDP helps consumers identify the products that best match their expectations among available alternatives. This component encompasses recommendation algorithms to filter a wide range of information. Accordingly, it enables recommendations of relevant products to the consumer according to their needs and criteria. Four tasks involved in this stage are as follows: determining the evaluative criteria, deciding alternatives to evaluate, valuing the performances of alternatives, and applying a decision rule. 
Consumers consider a maximum of six criteria and five products according to the consumer behavior literature [2-5]. With the multi-criteria product recommendation component, consumers gain support in considering more evaluative criteria. First, the component determines these criteria, which reflect features or characteristics consumers are interested in. It is preferable to have the criteria differentiated according to the product category. For example, evaluative criteria of a mobile phone and toothpaste are different. Hence, the component incorporates evaluative criteria according to the category of the product. Product taxonomies and ontologies provide the means to address the challenge of determining product categories. For example, GoodRelations [131] is an ontology used to specify the product category and product properties such as the screen size of a monitor, as well as delivery options, payment methods, and currency.

Second, this component determines the set of alternatives. Consumers typically consider only three to five product alternatives [132]. This component helps consider more products in the decision process. Again, product taxonomies help with the identification of alternative products.

Third, the multi-criteria product recommender component evaluates the performance of alternatives. This component uses public sources to collect information regarding product performances according to objective criteria. For example, price, display size, and the camera resolution of a mobile phone are available on the Internet. The information aggregator component of the information search stage also provides such required information to this component.

As the final task of the evaluation of alternatives stage, the proposed component applies a decision rule to choose the final product. Recommendation systems can be categorized as the single criterion and multi-criteria based systems [39]. For example, MobiShop [12], and LiveCompare [11] use only the price criterion to compare products. However, consumers typically use multiple criteria such as price, quality and performance. Therefore, a multi-criteria based approach is preferred within MobileCDP for recommending appropriate products, which meet the criteria of the consumer.

\subsection{Components for the Purchase Stage}

The outcome of the evaluation of alternatives stage is an intention to purchase: buy or do not buy. Once consumers evaluate the alternatives, they choose a particular product and brand in the fourth stage of the consumer decision process, the purchase decision [4]. Assistance regarding the payment method is mentioned as a function of a web based consumer decision support system in [105]. According to Assael [34], consumers may make up to five purchase sub-decisions as part of a purchase. These are brand, vendor, quantity, timing, and payment-method decisions. The proposed component for this stage assists consumers by facilitating these decisions. 


\subsubsection{Purchase Recommender Component}

This component provides recommendations by combining information regarding the user profile (e.g., suitable times for shopping), product prices, campaigns and discounts through the use of the credit, store, or loyalty cards, and multiple purchases to support the consumer in the purchase decision. The information aggregator component also supports this stage by providing information and reviews related to brands and vendors in order to facilitate brand and vendor decisions.

\subsection{Components for the Post-purchase Evaluation Stage}

After purchasing the product, the consumer begins to evaluate its performance during consumption. Depending on the relationship between the expectations of the consumer and perceived performance of the product, the outcome of post-purchase evaluation stage is satisfaction or dissatisfaction. This stage determines whether the consumer makes a complaint, purchases the product again, talks favorably or unfavorably about the product with others [133]. Moreover, consumer satisfaction is a critical factor on consumers' repurchase intentions, even with regard to virtual products [134].

As part of MobileCDP, the following three components are proposed to facilitate post-purchase evaluations of consumers:

\subsubsection{Post-purchase Review Component}

Recommendations and reviews of others significantly influence the consumer decision process [118]. The post-purchase review component facilitates the postpurchase evaluation stage by providing an interface to share complaints, satisfactions and reviews related to a product with other consumers. Since they have similar functionalities, My2cents [10] and APriori [64] can be used as modules implemented within the post-purchase review component.

\subsubsection{Post-purchase Feedback Component}

A feedback mechanism where consumers express their level of satisfaction or dissatisfaction with a particular purchase is mentioned as an important part of the post-purchase evaluation stage [119]. The post-purchase feedback component enables consumers to directly share their feedback with product manufacturers. This component also provides benefits for product manufacturers, since consumers feel more engaged with products if they are able to submit feedback about them [120].

\subsubsection{Post-purchase Consumer Support Component}

Consumer support via email is described as a function of a web based decision support system in [105]. This component provides a communication interface between consumers and vendors to enable a support mechanism. The consumer 
can send a support request to the vendor or manufacturer using this module, and check the status of the request, through the use of support tickets as one approach to provide this functionality. Moreover, this module includes a product registration mechanism to easily register a purchased product.

\subsection{Unifying Components}

The shopping list and the data collector components are the unifying components of the framework that are associated with all stages of the consumer decision process. Compared to other studies, these components are significant, since they enforce the holistic nature of MobileCDP by enabling information flow among all the components, as well as providing integration and orchestration of them.

\subsubsection{Shopping List Component}

The shopping list is an effective external memory storage mechanism for grocery purchasing [135]. Between $50 \%$ and $75 \%$ of consumers use a written shopping list and the majority of other consumers use a mental shopping list [136]. Research studies related to consumers' shopping habits with mobile solutions report that consumers rank "creating and arranging a shopping list" feature of a possible grocery shopping application as the highest priority $[67,106]$.

The shopping list component is not just a mobile form of a written shopping list; it also provides additional capabilities. While the consumer follows the stages of the consumer decision process, this component establishes an interface with other components, and transports information between the stages. For example, the consumer adds products to the shopping list in the problem recognition stage, and then the consumer gets information about these products in the information search stage. After that, the consumer evaluates alternatives of the products in the shopping list in the evaluation of alternatives stage. Next, the total cost of the shopping list is calculated in the purchase stage considering campaigns such as store, loyalty, and credit card campaigns. Once the purchase stage is completed, the products in the shopping list are recorded as part of past purchases. Finally, the consumer makes reviews about products in the past purchase list. As seen in this scenario, the data produced in a stage are made available and utilized in the following stages, through the use of the shopping list.

\subsubsection{Data Collector Component}

The data collector component provides data flow among proposed components of MobileCDP. It gathers data from components and provides data for them, such as product information, store information, past activities of the consumer, product lists and reviews. The data required by the components are collected implicitly or explicitly. The implicit data collection is collecting data while the user is interacting with the system in an implicit way. In this method, the system captures data while observing the activities of a user. For example, the priority of a criterion for a user 
can be identified using the historical data of this user; if the user always buys the cheapest product alternatives, this finding implies that the price criterion is the most important one for this user. In the explicit data collection, the user gives the required data in an explicit way. For example, asking users to sort criteria according to their preferences is an explicit data collection.

The data collector uses different input methods, such as barcode scanning to identify products. Receipt scanning is also an input method to collect data about transactions that includes store information, purchased products and their prices, the total cost of the transaction, the purchase date and time [137]. Free-text input is also used as a flexible data collection method, but it increases user annoyance. Moreover, the RFID approach of APriori [64], and the natural language input approach of Ma\$\$iv€ [67] can be used as input methods for data collection.

\subsection{Extensibility of the MobileCDP Framework}

The MobileCDP framework is built on a modular approach that allows incorporation of existing work in the literature into the framework, and extension of the framework by adding new components. The framework extension points are defined to provide extensibility of the MobileCDP framework. These extension points include common tasks that new components have to extend for providing new functionalities to the framework as well as extending functionalities of the existing components.

Similar to the component classification of the MobileCDP framework, the framework extension points are classified into two main types on the basis of functionality: stage extension points and unifying extension points. A stage extension point includes a common task that a new component has to implement for extending the framework for an individual stage of the consumer decision process. The stage extension points are based on the identified studies for each stage in the literature. For example, CreatelnternalStimuli and CreateExternalStimuli are the stage extension points of the problem recognition stage of the consumer decision process. At least one of them has to be implemented for designing a new component for the problem recognition stage. The identified stage extension points, corresponding stages, and brief descriptions are listed in Table 4.2.

The unifying extension points are responsible for integration of a new component with the unifying components. When designing a new component, at least one unifying extension point has to be implemented to define interconnection of this component with the unifying components. Then, the unifying components can provide integration of this component with the existing components. Table 4.3 lists the proposed unifying stage extension points, the corresponding unifying components, and brief descriptions of the extension points. 
Table 4.2 Stage extension points

\begin{tabular}{|c|c|c|}
\hline Stage Extension Point & Stage & Description \\
\hline CreatelnternalStimuli & Problem Recognition & Creating internal stimuli \\
\hline CreateExternalStimuli & Problem Recognition & Creating external stimuli \\
\hline MakelnternalSearch & Information Search & Performing internal search \\
\hline MakeExternalSearch & Information Search & Performing external search \\
\hline DetermineCriteria, & Evaluation of Alternatives & Determining evaluative criteria \\
\hline DetermineAlternatives & Evaluation of Alternatives & $\begin{array}{l}\text { Determining product } \\
\text { alternatives }\end{array}$ \\
\hline ValuePerformances & Evaluation of Alternatives & $\begin{array}{l}\text { Valuing performances of } \\
\text { product alternatives }\end{array}$ \\
\hline ApplyDecisionRule & Evaluation of Alternatives & Applying a decision rule \\
\hline RecommendBrand & Purchase & Brand recommendation \\
\hline RecommendVendor & Purchase & Vendor recommendation \\
\hline RecommendQuantity & Purchase & Quantity recommendation \\
\hline RecommendTiming & Purchase & Timing recommendation \\
\hline RecommendPaymentMethod & Purchase & $\begin{array}{l}\text { Payment method } \\
\text { recommendation }\end{array}$ \\
\hline MakeComplaint & Post-purchase Evaluation & $\begin{array}{l}\text { Making complaints about the } \\
\text { purchased product }\end{array}$ \\
\hline ShareSatisfaction & Post-purchase Evaluation & $\begin{array}{l}\text { Sharing satisfaction and } \\
\text { dissatisfaction }\end{array}$ \\
\hline MakeReview & Post-purchase Evaluation & $\begin{array}{l}\text { Making reviews about the } \\
\text { purchased product }\end{array}$ \\
\hline
\end{tabular}


Table 4.3 Unifying extension points

\begin{tabular}{lll}
\hline Unifying Extension Point & Unifying Component & Description \\
\hline Addltem & Shopping List & $\begin{array}{l}\text { Adding a new product to the } \\
\text { shopping list }\end{array}$ \\
\hline Removeltem & Shopping List & $\begin{array}{l}\text { Removing an item from the } \\
\text { shopping list }\end{array}$ \\
\hline RequestData & Data Collector & $\begin{array}{l}\text { Requesting data from the data } \\
\text { collector module }\end{array}$ \\
\hline SendData & Data Collector & $\begin{array}{l}\text { Sending data to the data } \\
\text { collector module }\end{array}$ \\
\hline
\end{tabular}

\subsection{Chapter Summary}

The proposed MobileCDP framework includes twelve components that support consumers in their activities, which are proposed through examining the relevant literature. These components are classified depending on their functionalities into facilitating and unifying components. Facilitating components target individual stages of the consumer decision process. These are the context-aware reminder and context-aware personal promotion components for the problem recognition stage; the experience recorder, review search, and information aggregator components for the information search stage; the multi-criteria product recommendation component for the evaluation of alternatives stage; the purchase recommender component for the purchase stage; and the post-purchase review, post-purchase feedback and post-purchase consumer support components for the post-purchase evaluation stage. Unifying components are responsible for integration and coordination among the facilitating components, which are the data collector and shopping list components. Each component is proposed based on the corresponding identified studies in the literature, as listed in Table 4.1. Due to the modular approach, the design allows incorporation of existing work in the literature into the framework, as well as extensions of the framework by adding new components. 


\section{CHAPTER 5}

\section{THE PARTICIPATORY SENSING APPROACH}

Rapid advances in information and communication technologies change the nature of consumer and business markets [57]. Consumers no longer act independently; they are increasingly connected with other consumers $[57,58]$. Since information is one of the most important elements of the consumer decision process, the rapidly increasing connectivity between consumers transforms shopping behavior and thinking ways of consumers by utilizing the collective information [58]. In addition, according to Wuyts, et al. [57], information sharing between consumers and using the collective information increase the efficiency and effectiveness of the decision process, and improves the quality of the decision.

The participatory sensing approach is beneficial for a system that requires usage of such collective information. Furthermore, this approach enables collecting some information that cannot be gathered without participation. For example, it is difficult to find grocery items' price and features, which consumers do not typically purchase online. The participatory sensing approach enables collecting such data through the contributions of the participants.

Therefore, we use the participatory sensing approach in the MobileCDP framework. The participatory sensing approach empowers MobileCDP by providing collective information that is required by each component of the framework to increase efficiency and effectiveness of the components, improve decision quality, and enable new functionalities, which are described in this chapter.

As mentioned in Chapter 2.2, the participatory sensing approach consists of four phases: collect, analyze, share, and actuate. In order to utilize the benefits of the participatory sensing approach, we design the MobileCDP framework to be compatible with each phase of the approach. In the collect phase, gathered data by consumers are sent to a back-end server, and stored in the mobile device of the participant. In the analyze phase, the collected data are analyzed in back-end servers and mobile devices. Then, in the share phase, analyzed data are displayed on the consumer's mobile phone, shared with other consumers, and used by all components of the MobileCDP. In a typical participatory sensing system, automated methods trigger actuations if they detect matches between analyzed data and 
predefined patterns. Similarly, in the actuate phase, the MobileCDP framework facilitates actions taken by consumers.

In summary, the participatory sensing approach provides required data for each component of the MobileCDP, and enables analyzing and sharing data to facilitate consumers' actions, as shown in Table 5.1.

\subsection{Participatory Sensing Support for the Components}

In the following subsections, first, each component of the MobileCDP framework is described in terms of the phases of the participatory sensing approach to show the compatibility of the component with this approach. Then, benefits of the participatory sensing approach are explained for each component.

\subsubsection{Context-aware Reminder}

Compatibility with Participatory Sensing:

Briefly, this component reminds the consumer a needed product, when this consumer attains proximity to the location of the store in the reminder settings. In the collect phase, the product to be reminded, the preferred location for the remainder, and the reminder expire date data are collected. Then, the context-aware reminder component periodically analyzes the current context (the location and time) of the consumer, and compares with previously recorded reminders in the analyze phase. If the component finds a match between the consumer's context and a recorded reminder, it shows a reminder message to the consumer in the share phase. After that, the consumer recognizes the problem, which is the need for the reminded product.

\section{Benefits of Participatory Sensing:}

In addition, the participatory sensing approach contributes a new functionality to the context-aware reminder component, which is the collaborative reminder concept. In this concept, a participant can share a context-aware reminder with another user to remind the need for a product to that user at the intended time and place.

\subsubsection{Context-aware Personal Promotion}

\section{Compatibility with Participatory Sensing:}

In the collect phase, the context-aware personal promotion component obtains information about the product to be promoted from the consumer, and information about current promotions from stores. In the analyze phase, this component periodically analyzes the current context and the collected promotion setting of the consumer, and compares them with the promotions offered by stores. If there is a match between the compared items, this component shows corresponding offers to 
the consumer in the share phase. Then, the consumer recognizes offered promotions about particular products, and decides whether he or she wants to purchase these products in the actuate phase.

\section{Benefits of Participatory Sensing:}

The participatory sensing approach brings two new features to this component. The first one is the collaborative promotion feature. Using this feature, a participant can share a promotion offered by a store with another user. Then, this user can request the same promotion from the store. The shared promotion includes general information about the promotion, such as the current and discounted prices of the promoted product. This promotion information does not include user-specific data, such as a promotion code that is produced for a specific user.

The second feature brought by the participatory sensing approach is the collaborative discount feature. Using this feature, the participants, who want to request a discount about a specific product, can collaboratively demand a special discount for the product. It is a similar concept with the "online group-buying auction", which is collecting enough consumer to generate an adequate volume of orders to create the basis for a lower transaction price [138].

\subsubsection{Experience Recorder}

\section{Compatibility with Participatory Sensing:}

The experience recorder component gathers the activities of the consumer, such as purchase transactions and product comparisons, in the collect phase. A transaction includes information about the purchase, e.g., the purchase time, store, purchased products and corresponding prices. In the analyze phase, information requirements of the consumer are analyzed, for example, whether the consumer needs information related to previously purchased products. Then, this component shows the previously collected information to the consumer in the share phase. Since the collected information is related to internal activities, it is used to perform the internal information search task in the actuate phase.

\section{Benefits of Participatory Sensing:}

Without the participatory sensing, collected data by this component are only used by the consumer who produces the data. However, utilizing the participatory sensing approach, this data can be used to bring new functionalities to the system. For example, the system can use purchase transactions of consumers to identify social neighbors for collaborative filtering, which is a method to identify consumers that have similar shopping preferences, and recommend the products they would like, as mentioned in Chapter 2.1.7. A very basic type of the collaborative filtering method is used by many e-commerce systems to recommend new products to users, such as "customers who bought this item also bought following items" recommendation. 


\subsubsection{Review Search}

Compatibility with Participatory Sensing:

In the collect phase, the review search component acquires reviews related to a product from online sources, such as social networking sites, blogs, discussion forums, online retailers' sites, and online review sites, as mentioned in Chapter 4.2.2. Then, in the analyze phase, this component analyzes collected information to find the relevant reviews about the current product. In the share phase, the review search component shows the relevant reviews to the consumer. In the actuate phase, the consumer performs the external information search task of the information search stage.

\section{Benefits of Participatory Sensing:}

Without the participatory sensing, this component only provides reviews collected from online sources. However, the participatory sensing approach enables sharing of reviews with other participants. In addition, analyzing reviews of different participants about a product provides an average score for the product.

\subsubsection{Information Aggregator}

\section{Compatibility with Participatory Sensing:}

In the collect phase, the information aggregator component gathers information from non-marketing and marketing controlled sources, as explained in Chapter 4.2.3. Then, in the analyze phase, this component classifies marketing and non-marketing controlled sources and filters product alternatives that are incompatible with the user's profile by analyzing the user's profile and product information. In the share phase, the information aggregator component presents filtered information to the consumer in a personalized manner. Finally, in the actuate phase, the consumer performs an external information search using the presented information.

Benefits of Participatory Sensing:

Utilizing the participatory sensing approach, the information aggregator component also collects data produced by other participants as non-marketing controlled sources. In addition, the participatory sensing approach enables simultaneous interaction with other consumers to search information. For example, a participant can ask opinions about a product to another participant by using this component.

\subsubsection{Multi-criteria Product Recommendation}

Compatibility with Participatory Sensing:

In the collect phase, the multi-criteria product recommendation component obtains inputs to apply a decision rule. These inputs include the evaluative criteria, product 
alternatives, performances of these alternatives according to the evaluative criteria, consumers' weights for the evaluate criteria, and criteria thresholds as explained in Chapter 5.3.1. Then, in the analyze phase, this component uses decision rules to generate a ranked product list from the set of alternatives. In the actuate phase, the consumer chooses a product from the ranked product list.

\section{Benefits of Participatory Sensing:}

The participatory sensing approach enables the collection of the required data for the multi-criteria product recommendation component. For example, prices and features of grocery products can be collected through the participatory sensing. In addition, the participatory sensing approach enables the collaborative filtering method for recommendations, which uses information about other participants. Moreover, this approach enables simultaneous interaction with other consumers to collaboratively evaluate product alternatives, similar to group decision-making.

\subsubsection{Purchase Recommender}

Compatibility with Participatory Sensing:

In the collect phase, the purchase recommender component gathers the shopping list of the consumer, user preferences (e.g., suitable times for shopping), product prices, campaigns, and discounts through the use of the credit, store, or loyalty cards, and multiple purchases. Then, in the analyze phase, this component applies optimization algorithms to find the cheapest store and store combination regarding the products in the shopping list. It presents the cheapest store and store combination to the consumer in the share phase, and the consumer makes the store and payment-method decisions in the actuate phase.

\section{Benefits of Participatory Sensing:}

Utilizing the participatory sensing approach, a campaign used by a consumer can be recommended to another consumer. This component can automatically detect campaigns from consumers' purchases, or a consumer can manually share the campaign information using the framework.

\subsubsection{Post-purchase Review}

\section{Compatibility with Participatory Sensing:}

In the collect phase, the post-purchase review component obtains reviews of the consumer about a product. In the analyze phase, information requirements of the consumer are analyzed, such as whether the consumer needs reviews about products. Then, the post-purchase review component shares the reviews of the consumer with other consumers in the share phase. Next, the consumer performs the external information task in the actuate phase. 
Benefits of Participatory Sensing:

The main function of this component is sharing review information with other consumers. Accordingly, this component inherently uses the participatory sensing approach to provide its main functionality.

\subsubsection{Post-purchase Feedback}

Compatibility with Participatory Sensing:

In the collect phase, the post-purchase feedback component gathers feedback of the consumer, which includes the level of the consumer's satisfaction or dissatisfaction. Then, this component finds the manufacturer related to the collected feedback, in the analyze phase. Next, the post-purchase feedback component shares the feedback with the corresponding manufacturer. This component helps manufacturers to improve consumers' satisfaction in the actuate phase.

\section{Benefits of Participatory Sensing:}

Collected data by the post-purchase review and post-purchase feedback components are different as implemented in the prototype (Chapter 6.8 and Chapter 6.9). By design, collected reviews are shared with consumers and collected feedback are shared with manufacturers. However, utilizing the participatory sensing approach for the post-purchase feedback component, consumers can use more information to evaluate products.

\subsubsection{Post-purchase Consumer Support}

\section{Compatibility with Participatory Sensing:}

The post-purchase consumer support component acquires the consumer's support request in the collect phase. Then, in the analyze phase, this component finds the manufacturer/vendor related to the support request. After that, it shares the support request with the relevant manufacturer/vendor, in the share phase. In the actuate phase, the manufacturer/vendor responses the support request of the consumer.

\section{Benefits of Participatory Sensing:}

This component shares support requests with manufacturers and vendors. Utilizing the participatory sensing approach, these support requests can be shared with other consumers. Then, these consumers can use the support request data, which usually include problems with a product, to evaluate the product. 
Table 5.1 Components of the MobileCDP framework regarding the participatory sensing approach

\begin{tabular}{|c|c|c|c|c|}
\hline Component & Collect & Analyze & Share & Actuate \\
\hline $\begin{array}{l}\text { Context-aware } \\
\text { Reminder }\end{array}$ & $\begin{array}{l}\text { Reminder } \\
\text { settings of the } \\
\text { consumer }\end{array}$ & $\begin{array}{l}\text { Whether the } \\
\text { current } \\
\text { context of the } \\
\text { consumer } \\
\text { matches with } \\
\text { the reminder } \\
\text { settings }\end{array}$ & $\begin{array}{l}\text { Shows a } \\
\text { reminder } \\
\text { message to } \\
\text { the consumer }\end{array}$ & $\begin{array}{l}\text { The consumer } \\
\text { recognizes the } \\
\text { problem }\end{array}$ \\
\hline $\begin{array}{l}\text { Context-aware } \\
\text { Personal } \\
\text { Promotion }\end{array}$ & $\begin{array}{l}\text { Promotion } \\
\text { settings of the } \\
\text { consumer, } \\
\text { offers from } \\
\text { stores }\end{array}$ & $\begin{array}{l}\text { Whether the } \\
\text { current } \\
\text { context of the } \\
\text { consumer } \\
\text { matches with } \\
\text { promotions }\end{array}$ & $\begin{array}{l}\text { Shows the } \\
\text { corresponding } \\
\text { offer to the } \\
\text { consumer }\end{array}$ & $\begin{array}{l}\text { The consumer } \\
\text { recognizes the } \\
\text { problem }\end{array}$ \\
\hline $\begin{array}{l}\text { Experience } \\
\text { Recorder }\end{array}$ & $\begin{array}{l}\text { Transactions } \\
\text { of the } \\
\text { consumer }\end{array}$ & $\begin{array}{l}\text { Information } \\
\text { requirements } \\
\text { of the } \\
\text { consumer }\end{array}$ & $\begin{array}{l}\text { Shows the } \\
\text { collected data }\end{array}$ & $\begin{array}{l}\text { The consumer } \\
\text { performs } \\
\text { internal } \\
\text { information } \\
\text { search }\end{array}$ \\
\hline Review Search & $\begin{array}{l}\text { Product } \\
\text { reviews from } \\
\text { online sources }\end{array}$ & $\begin{array}{l}\text { Which } \\
\text { reviews } \\
\text { correspond } \\
\text { to the current } \\
\text { product }\end{array}$ & $\begin{array}{l}\text { Shows the } \\
\text { corresponding } \\
\text { reviews }\end{array}$ & $\begin{array}{l}\text { The consumer } \\
\text { performs } \\
\text { external } \\
\text { information } \\
\text { search }\end{array}$ \\
\hline $\begin{array}{l}\text { Information } \\
\text { Aggregator }\end{array}$ & $\begin{array}{l}\text { Data from } \\
\text { online sources }\end{array}$ & $\begin{array}{l}\text { Classify and } \\
\text { filter } \\
\text { collected } \\
\text { data }\end{array}$ & $\begin{array}{l}\text { Shows the } \\
\text { relevant } \\
\text { product } \\
\text { information }\end{array}$ & $\begin{array}{l}\text { The consumer } \\
\text { performs } \\
\text { external } \\
\text { information } \\
\text { search }\end{array}$ \\
\hline $\begin{array}{l}\text { Multi-criteria } \\
\text { Product } \\
\text { Recommendation }\end{array}$ & $\begin{array}{l}\text { Inputs for } \\
\text { decision } \\
\text { making } \\
\text { algorithms }\end{array}$ & $\begin{array}{l}\text { Apply multi- } \\
\text { criteria } \\
\text { decision } \\
\text { rules }\end{array}$ & $\begin{array}{l}\text { Shows a } \\
\text { ranked list of } \\
\text { products }\end{array}$ & $\begin{array}{l}\text { The consumer } \\
\text { chooses the } \\
\text { final product }\end{array}$ \\
\hline
\end{tabular}




\begin{tabular}{|c|c|c|c|c|}
\hline $\begin{array}{l}\text { Purchase } \\
\text { Recommender }\end{array}$ & $\begin{array}{l}\text { Shopping list, } \\
\text { product } \\
\text { prices, } \\
\text { campaigns }\end{array}$ & $\begin{array}{l}\text { Apply } \\
\text { algorithms to } \\
\text { find the } \\
\text { cheapest } \\
\text { store / store } \\
\text { combination }\end{array}$ & $\begin{array}{l}\text { Shows the } \\
\text { cheapest } \\
\text { store/store } \\
\text { combination }\end{array}$ & $\begin{array}{l}\text { The consumer } \\
\text { makes store and } \\
\text { payment- } \\
\text { method } \\
\text { decisions }\end{array}$ \\
\hline $\begin{array}{l}\text { Post-purchase } \\
\text { Review }\end{array}$ & $\begin{array}{l}\text { Review of the } \\
\text { consumer } \\
\text { about a } \\
\text { product }\end{array}$ & $\begin{array}{l}\text { Whether the } \\
\text { consumer } \\
\text { need review } \\
\text { information }\end{array}$ & $\begin{array}{l}\text { Shares } \\
\text { reviews with } \\
\text { others }\end{array}$ & $\begin{array}{l}\text { The consumer } \\
\text { performs } \\
\text { external } \\
\text { information } \\
\text { search }\end{array}$ \\
\hline $\begin{array}{l}\text { Post-purchase } \\
\text { Feedback }\end{array}$ & $\begin{array}{l}\text { Level of } \\
\text { consumers' } \\
\text { satisfaction or } \\
\text { dissatisfaction }\end{array}$ & $\begin{array}{l}\text { Which } \\
\text { manufacturer } \\
\text { is related to } \\
\text { the feedback }\end{array}$ & $\begin{array}{l}\text { Shares } \\
\text { feedback with } \\
\text { the } \\
\text { manufacturer }\end{array}$ & $\begin{array}{l}\text { The } \\
\text { manufacturer } \\
\text { improves } \\
\text { consumers' } \\
\text { satisfaction }\end{array}$ \\
\hline $\begin{array}{l}\text { Post-purchase } \\
\text { consumer } \\
\text { Support }\end{array}$ & $\begin{array}{l}\text { The } \\
\text { consumer's } \\
\text { support } \\
\text { request }\end{array}$ & $\begin{array}{l}\text { Which } \\
\text { manufacturer } \\
\text { is related to } \\
\text { the support } \\
\text { request }\end{array}$ & $\begin{array}{l}\text { Sends support } \\
\text { request to the } \\
\text { manufacturer }\end{array}$ & $\begin{array}{l}\text { The } \\
\text { manufacturer } \\
\text { responses the } \\
\text { support request }\end{array}$ \\
\hline
\end{tabular}

\subsection{The Proposed Data Collection Method}

Automated data collection and minimized user annoyance are important for any participatory sensing application; accordingly, we focus on automated data collection by consumers. The data collector component accepts different input methods, such as barcode scanning and free-text input. Barcode scanning is mainly used for product identification. Free-text input is a flexible data collection method; however, it increases the user annoyance.

Recent developments related to mobile devices have led to an increase in their digital imaging capabilities as well as the emergence of new approaches in solving problems, such as automated data collection by using Optical Character Recognition (OCR) for automatic extraction of various information from images captured by mobile phone cameras $[12,139,140]$. In this research, we provide a receipt scanning method by using OCR and our proposed algorithms. Comparing with other input methods, receipt scanning by capturing an image of the receipt requires minimal user effort to collect data such as the store name, address, phone number, the purchase date and time, the list of the products and corresponding prices, the 
total price and taxes. These data are used in information search, evaluation of alternatives and purchase decision stages of the consumer decision process.

As a result of our research on receipt scanning, we propose an image-based receipt scanning method for automated data collection, which relies on OCR together with a supporting correction algorithm for removing erroneous information extracted from the image. Image-based OCR studies, which are enlisted in Chapter 2.3.3, try to deliver a solution to the problem of collecting information from store receipts solely through the application of OCR. Distinctively, our research adopts a methodology in which an additional knowledge-based correction algorithm (KBC) is used in order to extract common information in receipts and correct the erroneous parts, in support of the OCR method.

However, mobile phone captured images still have specific challenges such as uneven lighting, text skew, text misalignment, and focus loss, when compared with scanned images. In addition, the items in the image may be subject to the various external degradations, such as a document containing paper aging, stains, scratches and cracks [141]. These challenges further reduce the image quality and correct recognition performances of the mobile phone camera based OCR systems.

Accordingly, we propose a novel video-based method to improve the OCR accuracy, which combines the individual OCR outputs from multiple frames in a video. There are several occurrences of the same text in a document in different frames of its video sequence, and the OCR result for each occurrence may be different. For instance, a line may not be accurately recognized in the first frame, but it may be accurately recognized in the second frame. Therefore, inaccuracies in the OCR result in one frame can be compensated with a more accurate OCR result from another frame.

Considering these observations, the proposed method combines OCR outputs from multiple frames in order to extract and use the most reliable information presented in the individual frames. In our method, after a video of the document of interest is captured, OCR is employed for each frame in the video in order to extract the corresponding text. This operation is followed by the proposed text clustering based multi-frame integration (TB-MFI) algorithm, where the main principle is the clustering of "equivalent text lines" in individual frames according to a distance measure. Depending on their sizes, some of these clusters are eliminated by the algorithm, and the centroids of the remaining clusters are determined as the representative text lines in the document. Briefly, accuracy improvement is primarily obtained by integrating the best appearing text lines in low quality individual frames. In addition, if there is a knowledge base required by the KBC algorithm, the accuracy could be improved by correcting inaccurately extracted text using the KBC algorithm.

Detailed descriptions of the proposed image-based and video-based methods are given in the following sections. 


\subsubsection{Image-Based Method}

The image-based method is proposed to automatically collect data such as product and store information from receipts. This method consists of two steps. In the first step, the OCR process is applied to store receipt images captured by mobile phone cameras. Then, the knowledge-based correction algorithm (KBC) is applied to the output of the OCR process for increasing the accuracy of word and character recognition. The steps performed in OCR and KBC processes are shown in Figure 5.1 , and explained in detail below.

\section{Step 1 - Optical Character Recognition (OCR) process:}

The OCR approach is the most common method to extract characters in images, which enables automated data collection. Compared to the document scanner based OCR systems, various problems are encountered in the mobile device camera based OCR systems [142]. In the literature, there are studies such as OCRdroid [94], which address some of these problems by various image processing methods including binarization of the image and rotating the skewed image. The OCRdroid study shows that image processing methods cannot eliminate all of the mentioned problems. Accordingly, we proposed an automated knowledge based correction algorithm (KBC), which is based on prior information collected by using participatory sensing, language cues, and rules generated from the common structure of store receipts are used to address these problems.

The Tesseract OCR engine [95], which is known as one of the most accurate open source OCR engines [142, 143], is used to test and improve the OCR based image processing techniques applied in this research. Main reasons for selecting Tesseract OCR engine are its high accuracy as well as speed performance, and allowing improvement and editing due to its open source code availability. Moreover, Tesseract can be easily used in the scope of participatory sensing applications since it can run on mobile devices.

In this step, first, the OCR engine initially binarizes the image through adaptive thresholding as shown in Figure 5.1. Then, characters in the binarized image are identified with connected component analysis, and lines and words in the text are determined. Finally, the conversion process from the image to the text is completed through the recognition of the determined words.

The next step of the image-based method is the proposed knowledge based correction process (KBC). 


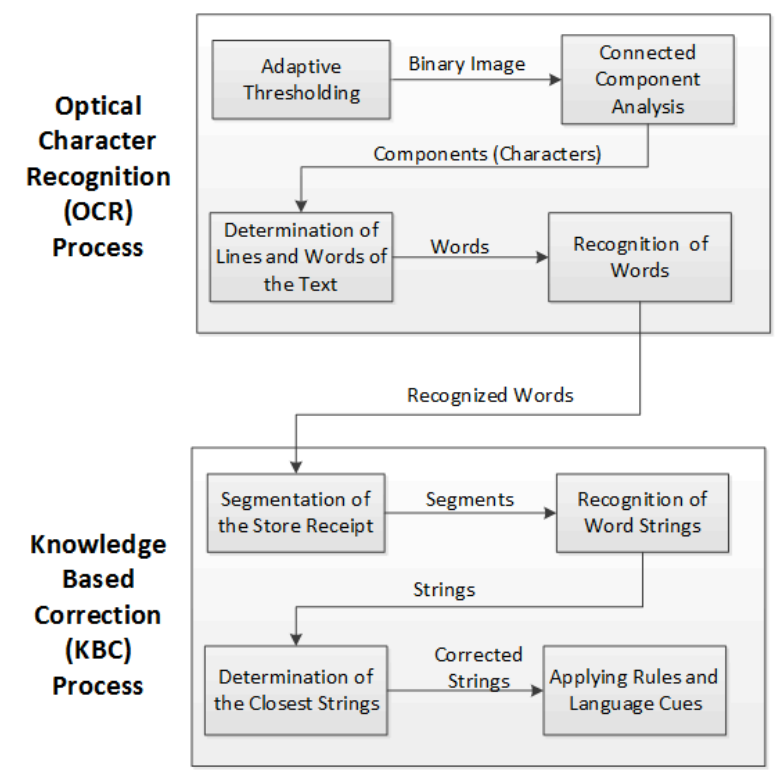

Figure 5.1 OCR and KBC processes applied to images for image-based method

\section{Step 2 - Knowledge-Based Correction (KBC) process:}

After image-to-text conversion, the proposed knowledge based correction process $(\mathrm{KBC})$ is applied to the extracted text. This process corrects erroneously recognized text pieces by using information collected through participatory sensing, such as product and store information.

In this step, first, a segmentation process is applied to the text obtained by OCR. These segments are i) store information segment ii) date, time, and receipt number segment, iii) product segment, and iv) total price and tax segment.

After the segmentation, strings in the segment are determined. In order to improve the accuracy, the KBC process corrects errors in store and product names through row-by-row comparison instead of word-by-word comparison. For example, correcting the "BIzIM PILAVL \$BULG" string is more effective than correcting the "PILAVL" word.

Next, the KBC algorithm is applied to rows (strings). The KBT algorithm uses different databases, which are specific to each segment, to correct errors. For example, "BIzIM PILAVL \$BULG" string in product segment is corrected and converted to "BIZIM PILAVLIK BULG" string by applying the KBC algorithm which uses product database.

We use the Levenshtein distance [144] in order to replace the wrong information with the right one in our correction algorithm. The Levenshtein distance is equal to the number of single-character edits required to change one string into the other. 
Let $x=x_{1} x_{2} \ldots x_{m}$ be the unique way to write string " $x$ " as a sequence of characters and let $y=y_{1} y_{2} \ldots y_{m}$ be the same for $y$. A single character edit of $x$ is one of the following:

- a deletion: the transformation of $x_{1} x_{2} \ldots x_{i-1} x_{i} x_{i+1} \ldots x_{m}$ to $x_{1} x_{2} \ldots x_{i-1} x_{i+1} \ldots x_{m}$ for some $1 \leq i \leq m$ ( $x_{i}$ is deleted).

- an insertion: the transformation of $x_{1} x_{2} \ldots x_{i} \ldots x_{m}$ to $x_{1} x_{2} \ldots x_{i} u \ldots x_{m}$ for some $1 \leq i \leq m$ and some character $u$ ( $u$ is inserted as a new character).

- a substitution: the transformation of $x_{1} x_{2} \ldots x_{i} \ldots x_{m}$ to $x_{1} x_{2} \ldots x_{i-1} u x_{i+1} \ldots x_{m}$ for some $1 \leq i \leq m$ and some character $u$ ( $u$ is substituted for $x_{i}$ ).

Then a complete edit from $x$ to $y$ is a combination of single character edits which input is $x=x_{1} x_{2} \ldots x_{m}$ and output is $y=y_{1} y_{2} \ldots y_{m}$. The length of an edit is the number of single character edits in a complete edit. The Levenshtein distance between $x$ and $y$, denoted as $\operatorname{lev}(x, y)$, is the length of the shortest complete edit from $x$ to $y$. For example, the Levenshtein distance between "CHE SE" and "CHEESE" words is 1 , and between "CHFFSE" and "CHEESE" words is 2.

The KBC algorithm also uses frequently encountered OCR errors while correcting character errors. As a result of experiments, the most frequent OCR errors are determined as given in Table 5.2.

Table 5.2 Frequently encountered OCR errors

\begin{tabular}{ll}
\hline Error Type & Substituted Characters \\
\hline Substitution of letters and digits & $\begin{array}{l}(0 \leftrightarrow O),(0 \leftrightarrow D),(\mid \leftrightarrow I),(2 \leftrightarrow Z),(5 \leftrightarrow S), \\
(6 \leftrightarrow G),(8 \leftrightarrow B)\end{array}$ \\
\hline Deficient recognition of the Turkish characters & $(C ̧ \leftrightarrow C),(O \leftrightarrow O),(U \leftrightarrow U),(S \leftrightarrow S)$, \\
\hline Deficient recognition of the other characters & $(O \leftrightarrow D),(R \leftrightarrow B),(R \leftrightarrow P),(E \leftrightarrow F)$, \\
\hline
\end{tabular}

Since the format of the date and time information in store receipt is well defined, this format information and frequently encountered OCR errors are defined as rule templates. In the last step of the KBC process, these rule templates are applied to 
text. For example, the time information that is recognized as $2 \mathrm{D}: 3 \mathrm{Z}$ is corrected automatically to $20: 32$ by rule templates.

\subsubsection{Video-Based Method}

In the image-based method, accuracy improvement is provided through utilizing prior information by KBC process. If the knowledge base required by the KBC algorithm is not available, the accuracy of the image-based method decreases.

Accordingly, we propose a video-based method, which improves the accuracy even with a low-quality video and without prerequisite knowledge. This method consists of a set of successive steps as shown in Figure 5.2. First, the OCR process, which is explained in the image-based method (Chapter 5.2.1), is applied to the extracted frames for obtaining text output of each frame after frames are extracted from the captured video. Then, equivalent lines are determined in order to form clusters, followed by an elimination of unwanted clusters. As the last step, the final text output is generated by combining the cluster centroids. These steps and the developed algorithms are explained next.

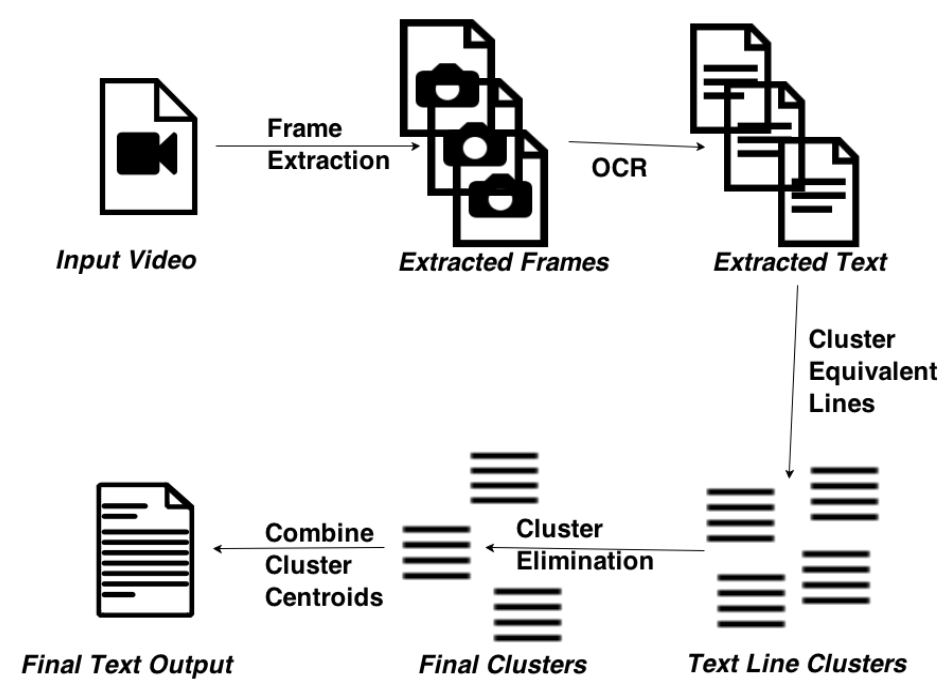

Figure 5.2 The proposed video-based approach

Text Clustering Based Multi-Frame Integration (TB-MFI):

The OCR step generates a text output as extracted from each individual frame of the video sequence, by using the Tesseract OCR engine. Figure 5.3(a) shows a sample frame, and Figure 5.3(b) is the corresponding OCR text output of this frame. As seen in Figure 5.3(b), the text output of a frame consists of several lines. 
The correctly extracted lines correspond to text lines in the store receipt. On the other hand, some of the extracted lines do not exist in the receipt; hence, they are not correctly extracted. For example, Line 1, Line 7 and Line 8 in Figure 5.3(b) exist in the extracted OCR output, but they do not exist in the original receipt. Moreover, there are inaccurately recognized characters in the lines extracted from the frame. For example, the correct text in the first line is "ŞOK MARKETLER T.A.Ş.", but the OCR engine extracts this line as "ŞOK MARKETLER T.A-FŞ", as seen in Figure 5.3.
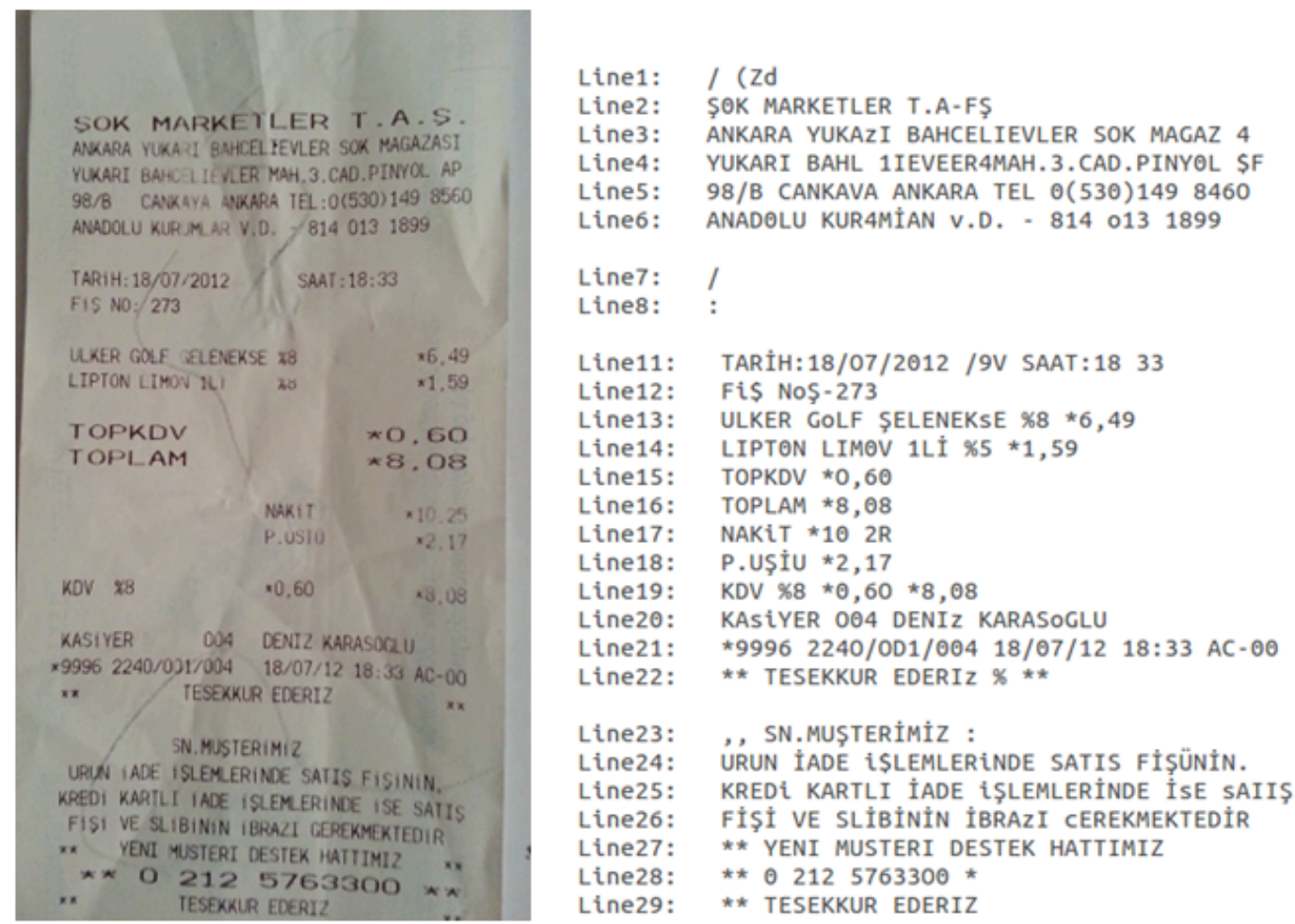

Figure 5.3 (a) A sample frame extracted from a video sequence (b) The OCR output of this frame

The proposed method reduces such problematic lines in order to increase the text recognition accuracy by using the following steps:

\section{Step 1 - Clustering equivalent lines in extracted frames:}

The same text line is visible in different frames of a video sequence, but the OCR output of this line may not be the same in different frames. For example, the first line in Figure 5.3(a) is extracted as "ŞOK MARKETLER T.A-FŞ" from one frame, and "ŞOK MARKETLER T.A-Ş" from another frame. While these extracted lines are different, the corresponding line in the captured receipt frame is the same. 
Hence, we refer to such lines as "equivalent lines" according to the Levenshtein distance [144] measure. Accordingly, the mathematical representation of the equivalency between two lines is given as:

$$
\operatorname{lev}\left(a_{m n}, a_{s t}\right)<k \Leftrightarrow a_{m n} \approx a_{s t} \quad \text { (Equation 5.1) }
$$

where $\operatorname{lev}\left(a_{m n}, a_{s t}\right)$ is the Levenshtein distance between lines $a_{m n}$ and $a_{s t}$, $\approx$ symbol denotes equivalency, and $k$ is the equivalency threshold as explained below. As explained in the image based method, the Levenshtein distance is equal to the number of single-character edits required to convert one string into another.

Our clustering algorithm includes the following sub-steps:

- Let Video $=x_{1} x_{2} \ldots x_{m}$ denote the sequence of the grabbed frames of the video stream, e.g., $x_{3}$ represents the 3rd frame of the video.

- Let Lines $=x_{11} x_{12} \ldots x_{1 n} x_{21} x_{22} \ldots x_{2 n} \ldots x_{m 1} x_{m 2} \ldots x_{m n}$ denote the rows in each frame, e.g., $x_{43}$ represents the 3rd row of the 4th grabbed frame.

1. Assign each line $x_{i j}$ to a separate cluster.

2. Denote the cluster element that has the highest number of occurrences as the centroid of the cluster. In other words, the centroid is the most frequently represented lines in this cluster.

3. Sort the cluster list as ascending according to $i$ and $j$ indexes of each cluster centroid. For example, the result of the first sorting process is $x_{11} x_{12} \cdot x_{13} x_{21} x_{22} \cdots$

4. Start with the first cluster and compare each cluster with all the following clusters in the cluster list. If cluster centroids of two clusters belong to the same frame, their comparison is skipped. For example, $x_{11}$ line is compared with $x_{21} x_{22} \ldots x_{2 n} \ldots x_{m 1} x_{m 2} \ldots x_{m n}$ first, and then $x_{12}$ line is compared with $x_{21} x_{22} \ldots x_{2 n} \ldots x_{m 1} x_{m 2} \ldots x_{m n}$.

5. The distance between two clusters is defined as the Levenshtein distance between the cluster centroids. If this distance is lower than the threshold $k$, these clusters are merged. This threshold is automatically calculated as a predefined ratio of string lengths of the centroids of the compared clusters. According to our experiments, the results are not sensitive to the threshold selection. 
We use centroid linkage clustering [145] in order to compare clusters. The distance between the two clusters is denoted as $\left\|c_{s}-c_{t}\right\|$ where $c_{s}$ and $c_{t}$ are the centroids of the clusters $s$ and $t$, respectively. Therefore, this step can be defined as follows:

$$
\text { if }\left\|c_{s}-c_{t}\right\|<k \text { then merge } s \text { and } t \quad \text { (Equation 5.2) }
$$

6. Repeat steps $2-5$ until no clusters are merged.

\section{Step 2 - Cluster elimination:}

In this step, the clusters that do not have any corresponding lines in the captured receipt are eliminated. As shown in Figure 5.3(b), Line 1 "/ (Zd" does not have a corresponding line in the captured receipt. Therefore, the OCR engine extracts this line inaccurately. The same erroneous line or a similar line such as "/ (zd" may appear in the text outputs of other frames, and in that case, they constitute a cluster. However, our experiments show that the occurrence of these erroneous lines is much less frequent than the real lines. Therefore, the proposed algorithm eliminates clusters that have a size (the number of the elements in the cluster) lower than the threshold $p$. This threshold is automatically calculated as a predefined ratio of the total number of frames used. Our experiments indicate that the results are not sensitive to the threshold $p$.

The following algorithm in Figure 5.4 is used for this step, and it returns the final list of clusters. In this algorithm, $n$ denotes the number of clusters in the cluster list, $C$ (i) denotes $i^{\text {th }}$ cluster of the cluster list, $M(C(i))$ denotes the size of the cluster $C(i)$, and $z$ denotes the number of clusters in the final cluster list.

$$
\begin{array}{ll}
\text { 1: } & z \leftarrow 0 \\
\text { 2: } & \text { for } i=0 \text { to } n-1 \text { do } \\
\text { 3: } & \text { if } M(C(i)) \geq p \text { then } \\
\text { 4: } & C(z) \leftarrow C(i) \\
\text { 5: } & z \leftarrow z+1 \\
\text { 6: } & \text { end if } \\
\text { 7: } & \text { end for } \\
\text { 8: } & \text { return } z \\
\text { 9: } & \text { for } i=0 \text { to } z-1 \text { do } \\
\text { 10: } & \text { return } C(z) \\
\text { 11: } & \text { end for }
\end{array}
$$

Figure 5.4 Cluster elimination algorithm 


\section{Step 3 - Generating final text output:}

In this step, the OCR outputs (lines) of the frames are combined to generate the final text output. The proposed algorithm includes the following sub-steps:

1. Determine the centroid of each cluster in the list. As described above, the centroid of a cluster is the element that has the highest number of occurrences.

2. Append these centroids, which represent the corresponding lines, to each other in order to generate the text output for the captured video sequence of the document.

\subsection{Proposed Multi-Criteria Product Recommendation Method}

As explained in Chapter 4.3.1, we implement a multi-criteria product recommender component in the prototype in order to support the evaluation of alternatives stage of the consumer decision process. We also propose a multi-criteria product recommendation method, which includes a meta-algorithm that uses the following data to make a recommendation: user information, product information, context data, social neighbors' data and recommendation parameters.

The main goal of the meta-algorithm is applying the appropriate multi-criteria decision algorithms (MCDA) to make a recommendation. Briefly, the meta-algorithm chooses these decision algorithms according to available amount of information. For example, if we do not have criteria thresholds, we cannot use sophisticated decision algorithms, such as the PROMETHEE and ELECTRE methods.

Evaluative criteria, user weights of these criteria, product alternatives, performance values of these criteria and multi-criteria algorithms are fundamental parts of our multi-criteria recommendation method. First four parts are inputs of the method and multi-criteria algorithms process these inputs and gives outputs such as overall performance of alternatives, ranks, binary comparison, outranking etc.

In this section, first, inputs of the proposed multi-criteria product recommendation method are described. Then, collection techniques of these inputs are explained. After that, the multi-criteria decision algorithms used by the meta-algorithm are defined. Lastly, we present the implementation of the proposed recommendation method.

\subsubsection{Inputs of the Proposed Method}

The multi-criteria product recommendation component facilitates evaluation of alternatives stage of the consumer decision-making process. As mentioned in Chapter 2.1.3, four tasks are involved in this stage: the consumer must determine the evaluative criteria to use for the purchase, the consumer must decide on 
alternatives to evaluate, the consumer must value the performance of alternatives, and the consumer must apply a decision rule to make the final product choice.

Respectively, the outputs of these three tasks are "evaluative criteria", "product alternatives" and "performance of alternatives". These parameters are inputs of the fourth task. In the fourth task, decision rules (procedures and strategies) are used to make the final choice from the set of alternatives. In addition to these parameters, the proposed multi-criteria recommendation component also uses "user weights for evaluative criteria", "criteria thresholds", and "preference direction" parameters to make more accurate product recommendation. All of these parameters are defined below.

\section{a) Evaluative criteria:}

Evaluative criteria specify the features or characteristics that the consumer wants or does not want and presents the objective attributes (such as chemical ingredients of toothpaste) and the subjective factors (such as prestige) of a product. Different products have different evaluative criteria. For example, chemical ingredients, sensitivity, and whitening factor are criteria of toothpaste; while CPU power, RAM size and HDD size are the criteria of a laptop. Price is a common criterion for both of them.

\section{b) Product alternatives:}

As mentioned before, after a successful information search (the second stage of the consumer decision making process), the consumer views a group of products (according to Dibb, et al. [133], the consumer's evoked set) as possible alternatives.

\section{c) Performances of alternatives:}

Performances of alternatives are given values to the criteria of alternatives. For example, the CPU power of the notebook is $2.1 \mathrm{GHz}$, the display size of the mobile phone is 5.1", and the price of the toothpaste is 11.5 TL. Our participatory framework collects performances of alternatives from participants, manufacturers, and vendors. However, especially for subjective criteria, information collected from manufacturers and vendors may be biased. Therefore, opinions of participants may be more distinguished for determining the performances of alternatives.

d) User weights for evaluative criteria:

Evaluative criteria may differ in their importance. For example, price may be the most important criterion in some decisions and quality may be the most important one in some other decisions. The importance of evaluative criteria depends on situational factors, individual factors and host of the product [4]. Therefore, the importance of evaluative criteria reflects weights of user preferences. 


\section{e) Criteria thresholds:}

Some complex multi-criteria decisions algorithms require additional parameters to compute a recommendation, which are called "criteria thresholds". The discrimination (indifference and preference) thresholds and the veto threshold are the most common criteria thresholds.

1) Indifference: If the difference between performances of two alternatives is lower than this threshold, the user does not have preference between two product alternatives for a given evaluative criterion.

2) Preference: If the difference between performances of two alternatives is above this threshold, the user prefers one alternative to another for a given evaluative criterion. Preference threshold justifies the preference in favor of one of the two alternatives.

3) Veto: If the difference between performances of two alternatives is above this threshold, the user "definitely" prefers one alternative to another for a given evaluative criterion. Suppose that user prefers the product $A$ to the product $B$ for four criteria with respect to preference thresholds, but the user prefers the product $B$ to the product $A$ for one criterion with respect to the veto threshold; then the user prefers product $B$.

4) Preference Direction: Each criterion of alternative set must be either associated with a maximum ( $\max )$ preference direction or a minimum ( $\min$ ) preference direction.

- If the preference on an alternative increases when the performance increases for a given criterion, this criterion is associated with the maximum (max) preference direction. For example, preference on an alternative increases when quality increases. Therefore, the quality criterion is associated with the "max" preference direction.

- If the preference on an alternative decreases when the performance increases for a given criterion, this criterion is associated with the minimum $(\mathrm{min})$ preference direction. For example, preference on an alternative decreases when the price increases. Therefore, the price criterion is associated with the "min" preference direction.

\subsubsection{Data Collection for Multi-criteria Recommendation}

The following techniques are designed to collect required data by the recommendation method. Using these techniques, data can be collected implicitly or explicitly. The implicit data collection is collecting data while the user interacting with the system in an implicit way. In this method, the system captures data while observing the behavior of a user. For example, if a user returns a product that means he or she is not satisfied with this product. The explicit data collection is done 
by explicitly entering data by the user. Filling a product review form is an example of explicit data collection.

Collecting user weights and criteria thresholds:

User weights for evaluative criteria and criteria thresholds (indifference, preference and veto) are important parameters for the meta-algorithm, which are described in Chapter 5.3.1. These parameters can be collected explicitly or implicitly.

In explicit data collection, users can directly submit criteria weights and thresholds regarding their preferences. Or, instead of directly asking weight and threshold values, the system can ask indirect questions (such as pairwise comparison questions) and calculate answers according to the answers using the AHP method suggested by Saaty [146].

In implicit data collection, the criteria weights and thresholds may be calculated from transactions of the user, which are collected by the data collector component of the framework mentioned in Chapter 4.6.2. For example, if the user frequently purchases a product that has a low price and low quality, the system can infer that the weight of the price is more important than the weight of quality. Another implicit data collection technique is the calculation of weights according to the social neighbors' data using the collaborative filtering methods [39].

Completing the missing data:

All of the required data by the multi-criteria decision algorithms may not be collected. We design the following five successive steps to complete missing data:

Step 1 - Asking questions to users: The most direct and convenient way to complete the missing data is asking questions to users. For example, if we do not have the weight of the price criterion of toothpaste, we can ask some questions to determine the weight of this criterion. Directly asking user weights for evaluative criteria, such as "what is the weight of price criteria for you", does not give appropriate weights [146]. On the other hand, the pairwise questions used in the AHP method, such as "is criterion $X$ is more important than criterion $Y$ " are more straightforward and appropriate for the user [146].

The AHP method requires $n(n-1) / 2$ pairwise comparison to precisely calculate user weights for $n$ criteria [146]. For example, 10 pairwise comparisons are required to calculate weights of five evaluative criteria. Predictably, asking questions may disturb users. Accordingly, the meta-algorithm can optimize questions. For example, if prices of alternative products are too close, we can ignore the price criterion for the multi-criteria decision making. Then, 6 pairwise comparisons are sufficient instead of 10 comparisons.

If the user does not answer all the required questions, the system passes to the second step. 
Step 2 - Getting global values of the user: Global values mean aggregated values of a user. For example, if we know the weight of the price criterion for some products, these weights can be aggregated by a function (e.g., average) to calculate the global weight of the price criterion. Using global values of the user is another way to complete the missing data. For example, if the weight of the price criterion of toothpaste is missing for a user, we can use the global weight of the price criterion.

Step 3: Getting missing values from social neighbors: The meta-algorithm also uses collaborative filtering methods to complete missing data. For example, if the weight of the price criterion of toothpaste is missing for a user, we can use the aggregated weight of social neighbors.

Step 4: Calculate/predict missing values: If all of the required data cannot be collected after the previous three steps, the meta-algorithm can calculate missing values using the known values. For example, if we know $x$ of $y$ criteria weights and sum of them is $z$, the meta-algorithm can assign $(1-x) /(y-x)$ to missing $y-x$ criteria weights.

\subsubsection{Multi-criteria Decision Algorithms}

In this section, multi-criteria decision algorithms used in the proposed multi-criteria recommendation method are explained.

\section{Weighted Sum method:}

The weighted sum method is the simplest and most common multi-criteria decision making (MCDM) method [147]. Suppose that we have $m$ product alternatives and $n$ evaluative criteria. A denotes alternative set and $C$ denotes criteria set. Next, suppose $w_{j}$ denotes the weight of the criterion $C_{j}$ and $a_{i j}$ is the performance value of the alternative $A_{i}$ according to the criterion $C_{j}$. Then, the overall performance of alternative $A_{i}$, denoted as $A_{i}^{\text {WSM-score }}$, is defined as follows:

$$
A_{i}^{W S M-s c o r e}=\sum_{j=1}^{n} w_{j} a_{i j}, \text { for } i=1,2,3, \ldots, n \quad \text { (Equation 5.3) }
$$

We need the alternative set, criteria set, alternative performances and user weights to calculate weighted sum scores of products.

AHP method:

Analytic Hierarchy Process (AHP) is an MCDA method [146]. Even though quantitative weights are not available, AHP can convert answers of pairwise questions (is criterion $X$ more important than criterion $Y$ ) to user weights of evaluative criteria. AHP decomposes a multi-criteria decision making problem to its components and constructs criteria hierarchies. AHP also supports the group decision making. 


\section{ELECTRE methods:}

ELECTRE stands for Elimination and Choice Translating Reality. ELECTRE methods are a family of MCDA methods. The first ELECTRE method evolved into ELECTRE I, ELECTRE II, ELECTRE III, ELECTRE IV, ELECTRE IS and ELECTRE TRI [148].

ELECTRE methods are based on "concordance" and "discordance" indices. ELECTRE I, ELECTRE II and ELECTRE IV use "simple concordance" index while ELECTRE III, ELECTRE IS and ELECTRE TRI use "complex concordance" index. The simple concordance index is calculated with criteria weights. On the other hand, the complex concordance index uses indifference and preference thresholds in addition to criteria weights.

ELECTRE II and ELECTRE IV use "binary discordance" index while ELECTRE III and ELECTRE TRI use "fuzzy discordance" index. ELECTRE I and ELECTRE IS do not use discordance index to calculate outranking value. Both veto and preference thresholds are required to calculate fuzzy discordance while the veto threshold is sufficient to calculate binary discordance.

A partial ranking is calculated in ELECTRE I method. The output of this method is a set of promising alternatives. A rank of alternatives is constructed in ELECTRE II method. An outranking degree is calculated in ELECTRE III, which is a more sophisticated and complicated method than other methods [149]. In the proposed meta-algorithm, we use ELECTRE III method.

PROMETHEE methods:

PROMETHEE methods are another MCDA method family [150]. Each PROMETHEE method has improvements over predecessors. Briefly;

- PROMETHEE I: partial ranking

- PROMETHEE II: complete ranking

- PROMETHEE III: ranking based on intervals emphasizing indifference

- PROMETHEE IV: continuous set of possible alternatives

- PROMETHEE V: MCDA including segmentation constraints

The meta-algorithm requires a complete ranking; therefore, it uses PROMETHEE II method in the PROMETHEE family. 


\subsubsection{Implementation of the Proposed Method}

We implement the proposed multi-criteria product recommendation method. In our implementation, the multi-criteria decision algorithms are applied by using the Decision Deck's XMCDA web services [151]. Implementation of the proposed method shows a ranked list of the product alternatives by following the below tasks as depicted in Figure 5.5.

1. The intended product category is identified by scanning of the barcode on the product package or label.

2. The system determines product alternatives by using the product category and product taxonomy.

3. The system determines the criteria of the product category.

4. The system shows the calculated criteria weights to user, and allows changes.

5. The system determines performances of alternatives such as price and quality, by using the product database.

6. The system acquires and calculates criteria thresholds.

7. The system creates five XML files, which consist of criteria names, names of product alternatives, criteria weights, criteria thresholds, and performances of alternatives datasets (a file for each dataset).

8. The system sends the created XML files to the web service. We used the Decision Deck's XMCDA web services [151] in order to calculate overall scores of product alternatives.

9. The web service calculates overall scores according to the selected multicriteria algorithms, and sends an XML file for each algorithm to the mobile device. An XML file consists of the overall performances of alternatives according to the corresponding multi-criteria decision algorithm, such as ELECTREE III or PROMETHEE II.

10. The system converts the received $X M L$ files, and then the resulting scores for the product alternatives are shown in a ranked list on the screen of the mobile device. 


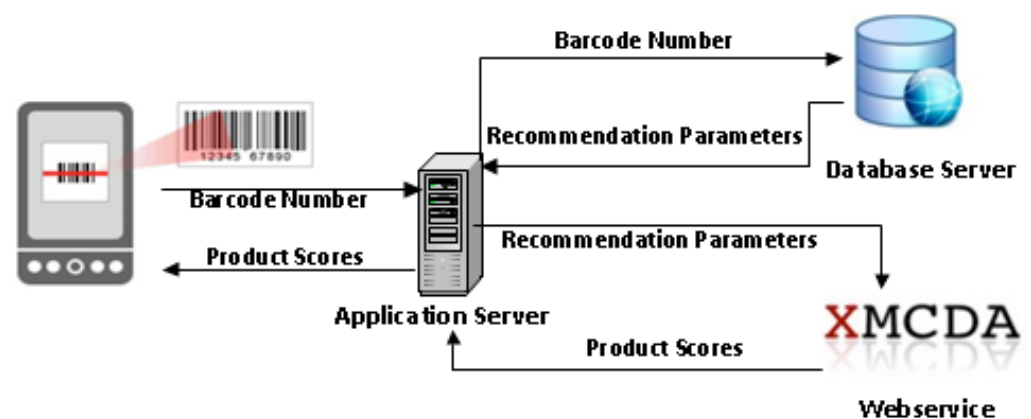

Figure 5.5 Implementation of the proposed multi-criteria recommendation method

\subsection{Chapter Summary}

This chapter presents how the participatory sensing approach reinforces the MobileCDP framework. As supplemented with the participatory sensing approach, the MobileCDP framework incorporates collect, analyze, share and actuate phases of a typical participatory sensing system. Data are collected, analyzed and shared by mobile phones of participants, and produced information is used to actuate the participant, while following the consumer decision process. The participatory sensing approach brings new functionalities to each component of the MobileCDP framework as summarized in Table 5.3. We also proposed automated data collection methods, which are image-based and video-based methods, to provide data for the framework. In addition, the proposed multi-criteria product recommendation method is presented in this chapter. 
Table 5.3 Benefits of the participatory sensing approach

\begin{tabular}{ll}
\hline Component & Benefits of Participatory Sensing \\
\hline Context-aware Reminder & Collaborative reminder \\
\hline $\begin{array}{l}\text { Context-aware Personal } \\
\text { Promotion }\end{array}$ & Collaborative promotion, collaborative discount \\
\hline Experience Recorder & Identifying social neighbors, collaborative filtering \\
\hline Review Search & Utilization of reviews of other consumers \\
\hline Information Aggregator & $\begin{array}{l}\text { Utilizing data produced by other consumers, } \\
\text { simultaneous information search }\end{array}$ \\
\hline Multi-criteria Product & Collection of recommendation input, collaborative \\
Recommendation & filtering, simultaneous product evaluation \\
\hline Purchase Recommender & Utilization of campaigns used by other consumers \\
\hline Post-purchase Review & Sharing review information with other consumers \\
\hline Post-purchase Feedback & $\begin{array}{l}\text { Utilization of feedback information for other } \\
\text { consumers }\end{array}$ \\
\hline Post-purchase Consumer & $\begin{array}{l}\text { Utilization of support request information for other } \\
\text { consumers }\end{array}$ \\
\hline Support &
\end{tabular}




\section{CHAPTER 6}

\section{PROTOTYPE}

A prototype mobile application is designed and implemented based on MobileCDP. It is deployed and tested on different Android mobile phones such as Samsung Galaxy S3, HTC Desire HD, and Motorola Moto G.

As depicted in Figure 6.1, the prototype system has a client-server architecture consisting of a mobile application that includes developed modules, a back-end server that runs the server application, and a database server that includes the databases. The mobile application can access the mobile device services; for example, the camera service to provide the barcode scanning function, and the local database to cache data. The mobile application communicates with the back-end server over the mobile device's network connection to exchange information. The back-end server accepts requests from the mobile application, processes them, and sends these queries to the database server. After that, the database server sends the requested data to the back-end server to be delivered to the mobile application.

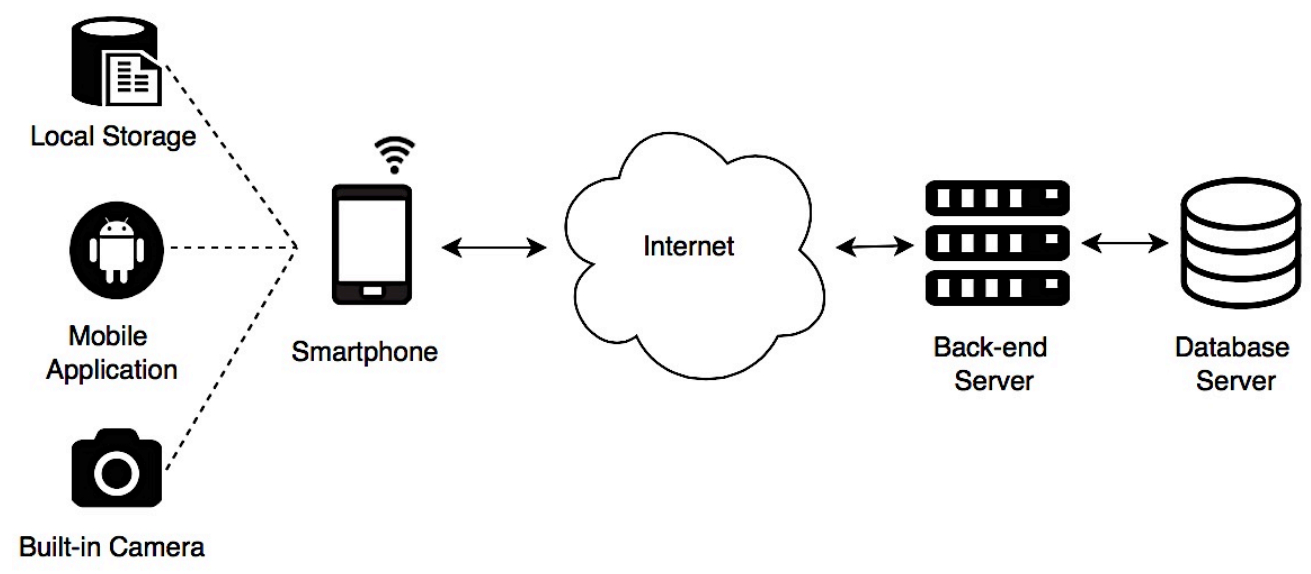

Figure 6.1 Client-server architecture of the prototype 
Figure 6.2 presents consumer activities and the facilitating modules in the prototype based on a sample scenario. Briefly, the user sets reminder and promotion options, by using the context-aware reminder and the context-aware personal promotion modules, respectively. The experience recorder module records the activities of the user, which consist of the purchased products, reviews and feedback; and shows this information to the user in a page. The review search module provides reviews of other users and detailed information about a product, which are provided by the information aggregator module. The multi-criteria product recommender module helps eliminate inappropriate alternatives, according to the user's criteria. The purchase recommender module calculates the total cost of the different purchase combinations of the products on the shopping list. The post-purchase review module provides sharing reviews of a product with other users, the post-purchase feedback module supports sharing the user's level of satisfaction or dissatisfaction about a particular product with product manufacturers, and the post-purchase consumer support module helps consumers send support requests to product manufacturers and vendors.

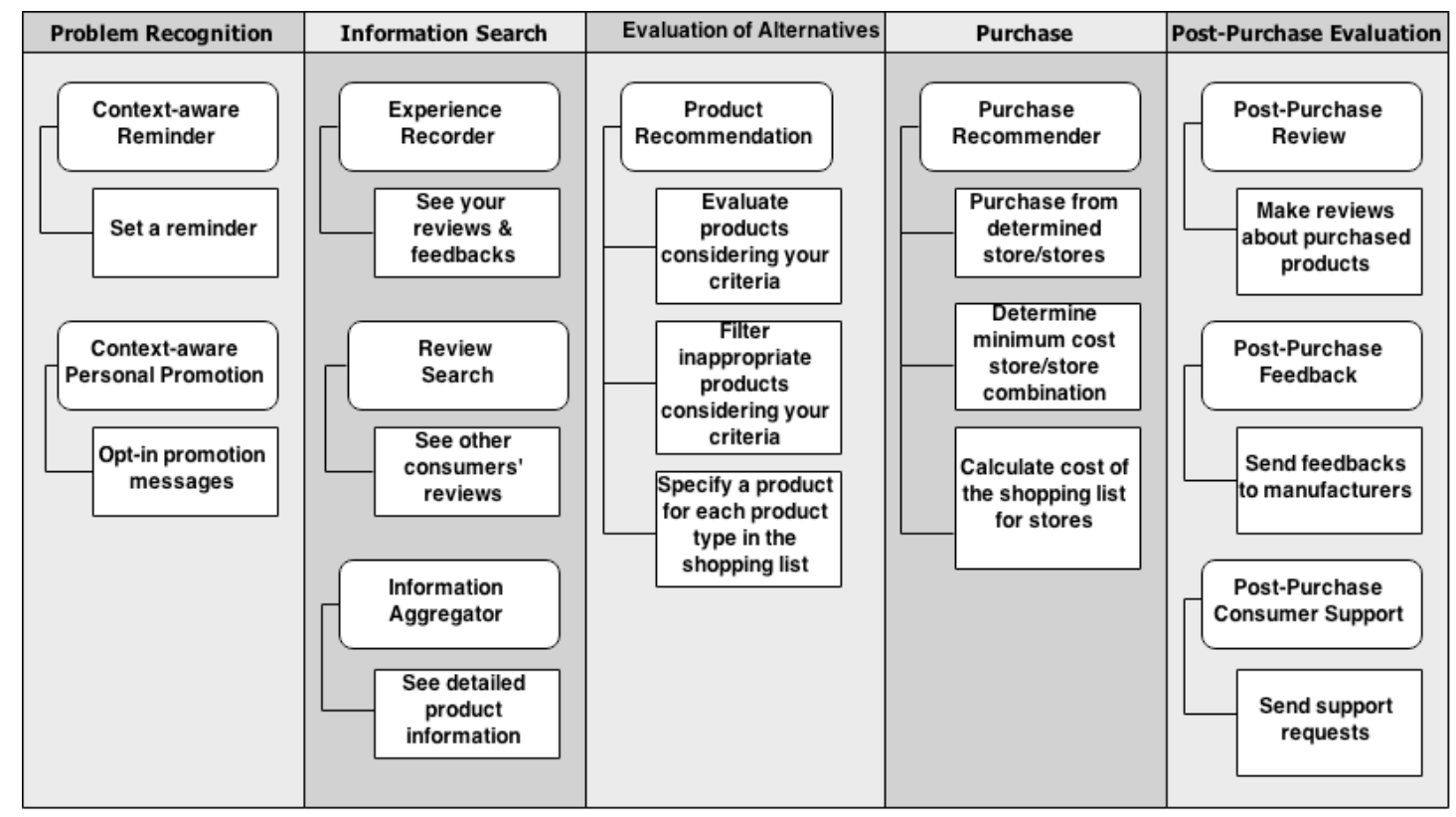

Figure 6.2 Consumer activities in a sample scenario and corresponding modules in the prototype

As a unifying module, the shopping list module integrates other modules. For example, the user can create reminder and promotion settings while adding a product to the shopping list. When a user selects a product in the shopping list, the information aggregator module shows detailed information about the product. The user filters and eliminates product alternatives in the shopping list using the multicriteria product recommender module, and the purchase module calculates the total 
cost of the items in the shopping list. The other unifying module, the data collector, supports coordination of data exchange of other modules by providing collection and dissemination functionalities. These modules are described in more detail in the following sections.

\subsection{Context-aware Reminder Module}

In the prototype application, users can add a reminder by choosing a reminder expiration date and store while adding a new item to the shopping list. Then, a reminder, which consists of the user identifier, product identifier, reminder duration, and the selected store, is saved to the database. If the user's current context matches any reminder in the database, which means the user is in the vicinity of the store before the reminder expiration date, the context-aware reminder module shows an alert to the user about the product.

\subsection{Context-aware Personal Promotion Module}

Similar to the context-aware reminder module, users can activate "get promotions" option while adding a new item to the shopping list. The promotion data, which are recorded in the database, include the user identifier, product identifier, store identifier, and promotion information. This module shows the promotion information to the user when the user's current context matches the promotion data. The context-aware reminder module works based on the opt-in principle; hence, promotions are not sent without the consent of the user. Users can list which items are selected for promotional messages, or remove their consents.

\subsection{Experience Recorder Module}

This module records the activities of the user, such as purchased products, reviews and feedback. These activities are shown in the "My History" screen under three tabs as shown in Figure 6.3. In the "My Purchases" tab, the user sees purchased products in a list-view with brief purchase information such as the purchase date, store and price. The "My Reviews" tab includes the user's reviews of products in a list-view. This tab also contains brief information about the reviews such as the review date, review title, rating value, and if it is recommended or not by the user to others. In the "My Feedback" tab, the user sees his or her earlier feedback to product manufacturers as a list. Product picture, name, feedback date, satisfaction level, recommendation state, and comparison with other products are given in this list-view. 


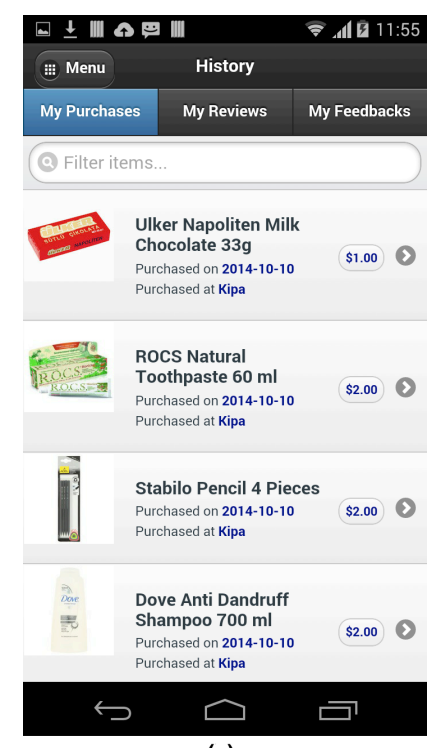

(a)

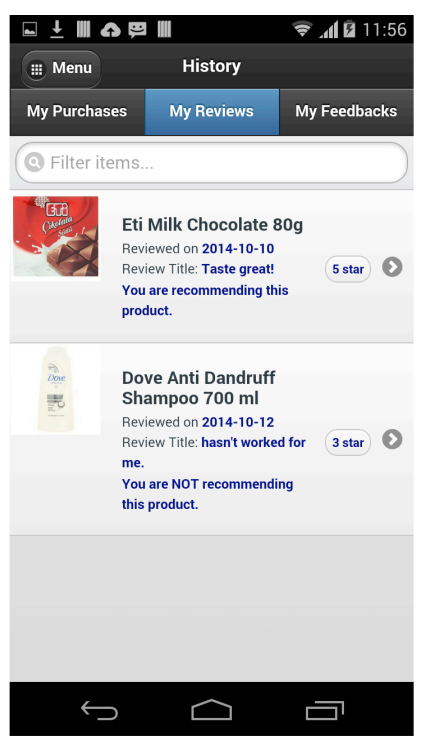

(b)

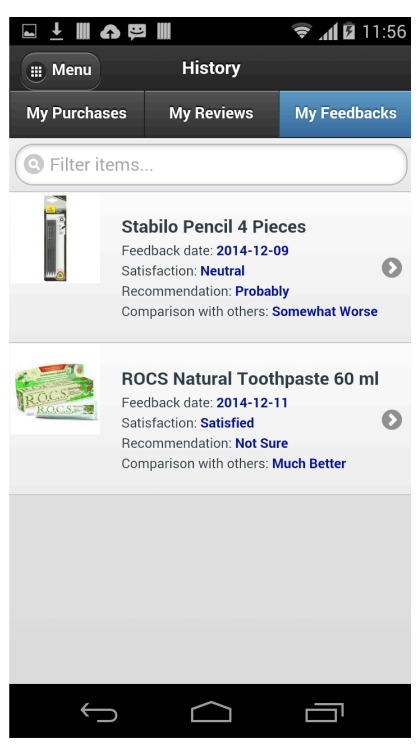

(c)

Figure 6.3 The screens of the Experience Recorder module: (a) My Purchases, (b) My reviews, and (c) My Feedbacks

\subsection{Review Search Module}

Using this module, the user searches for reviews of a product from the product list, or scan the barcode of the product using the smartphone's camera. Moreover, the "product details" screen also provides reviews of the selected product. A review includes the five star rating given for the product, the review title, the review date, the reviewer's username (if the reviewer allows), and the opinions of the reviewer. Since reviews occupy a large screen space for display, they are shown in collapsible blocks.

\subsection{Information Aggregator Module}

This module provides detailed information about the selected product. Common information given about products includes the product's thumbnail, name, average rating, average price, minimum price, minimum price store, unit price, country of origin, manufacturer, description, and reviews as seen in Figure 6.4. Moreover, detailed information about products is given according to the product type. For example, ingredients, allergens and nutrition information are given for food products. Also, this module includes a "Product List" screen, which lists all products with average price information, and provides a free-text search feature. Price information in the database includes the product identifier, store identifier that sells the product, and the price of the product for this store. The average price of a product is calculated using the available price information in the database. 


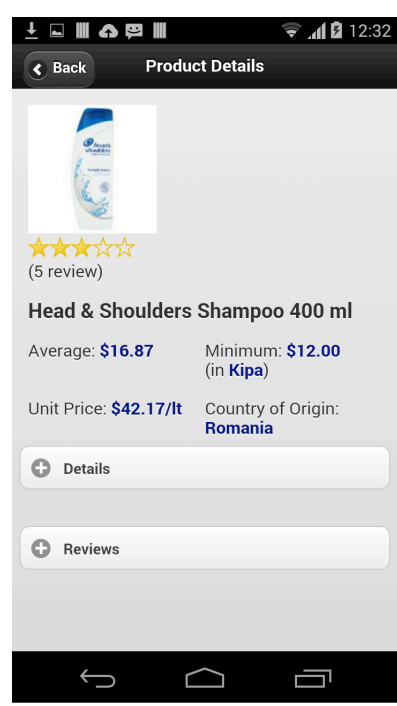

(a)

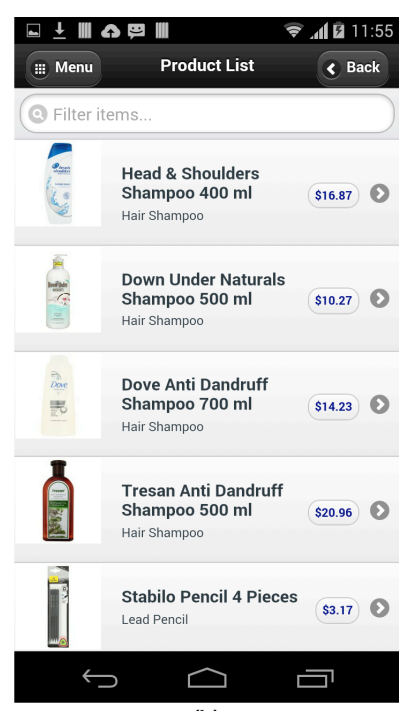

(b)

Figure 6.4 The screens of the Information Aggregator module: (a) Product Details, and (b) Product List

\subsection{Multi-Criteria Product Recommender Module}

The product recommendation module facilitates evaluation of alternatives stage by showing information about product alternatives, and helping in the elimination of inappropriate alternatives. As seen in Figure 6.5, "Product Alternatives" screen includes a filter section at the top, and a dynamic list-view below the filters, which consists of product alternatives.

The filter section includes a price range slider. The user can change the minimum and maximum prices using the slider. When the minimum or maximum price is changed, only the products in the selected price range remain in the dynamic listview. The filter section also includes elimination checkboxes that are used to eliminate products with unwanted features. These features vary according to product type. For example, a milk chocolate includes "allergens", "additives", and "sweeteners" filters, but a shampoo includes "chemical" and "toxic" filters. If a user chooses to remove "sweeteners", products that contain sweeteners disappear from the dynamic list-view. Therefore, the user easily filters inappropriate products by using filters, and instantly sees the effects of his or her preferences on the dynamic product alternatives list without changing the screen. 


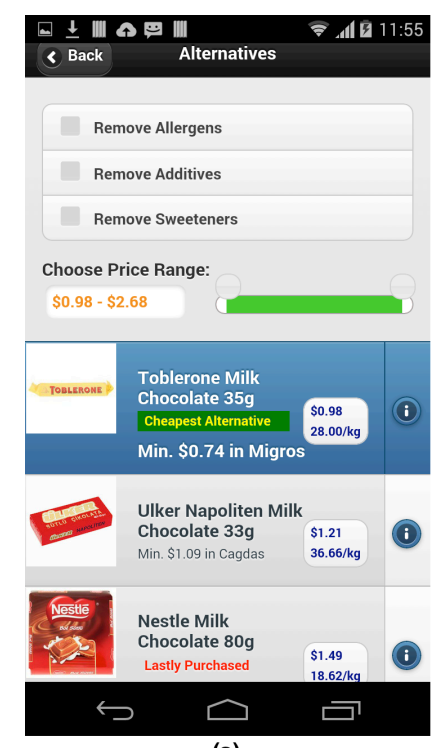

(a)

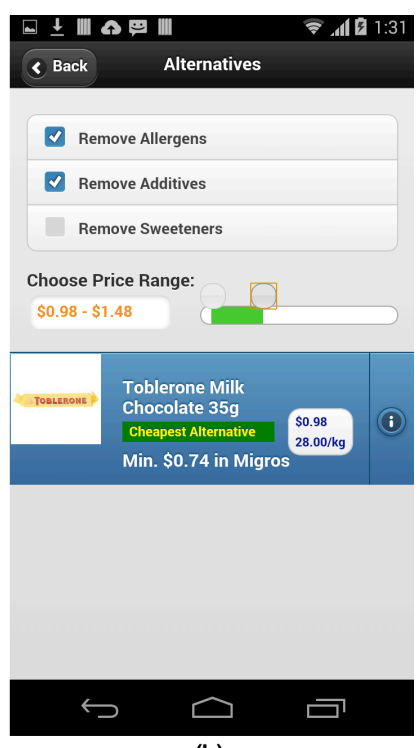

(b)

Figure 6.5 (a) Product alternatives screen (b) Product alternatives screen after selecting some filters

As seen in Figure 6.5(a), each product alternative is included as a row in the dynamic list-view, and each row consists of a thumbnail of the product, the minimum price and the corresponding store, the average price and the automatically calculated unit price. The user can also see the detailed information about the product by selecting it. Moreover, product alternatives with distinguishing features are indicated such as "the cheapest alternative" and "most recent purchase". If the user selects an unwanted feature in profile such as an indication of gluten allergy, product alternatives that have this feature are marked clearly to warn the user.

\subsection{Purchase Recommender Module}

This module supports the purchase decision by calculating the total cost of the different purchase combinations of products on the shopping list. The total cost includes product prices and the transportation cost to the store. Transportation costs are roughly estimated using the distance. Each store, and store combinations are shown with their total costs, as seen in Figure 6.6(a) and Figure 6.6(c), respectively. In the "stores" list-view, shown in Figure 6.6(b), users see the products, corresponding prices and the transportation cost. Users also see prices of a product at different stores by selecting a product name. The recommended store combination to obtain the minimum-cost is displayed in the "combination of stores" list-view, as depicted in Figure 6.6(c). The user also sees the products and corresponding prices by selecting a store combination in the same screen. The purchase recommender module highlights the minimum-cost store and minimumcost store combinations, as shown in Figure 6.6(a) and Figure 6.6(c), respectively. 


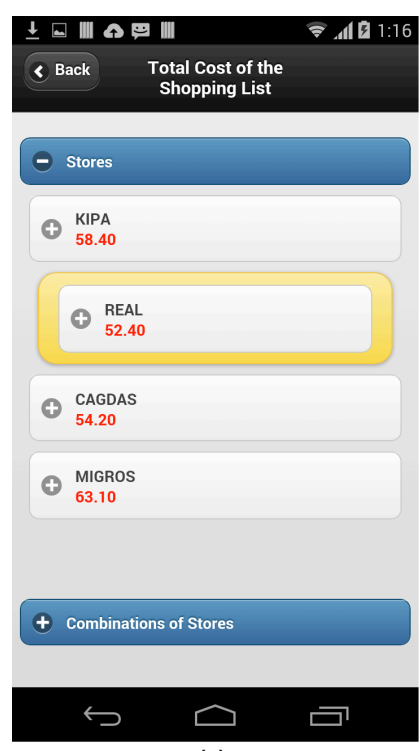

(a)

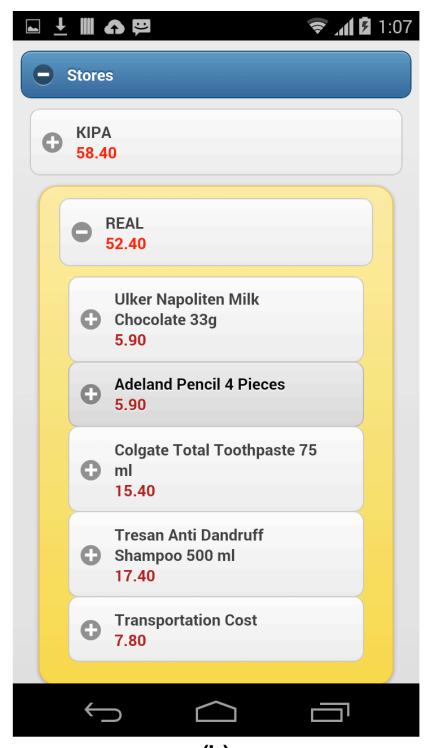

(b)

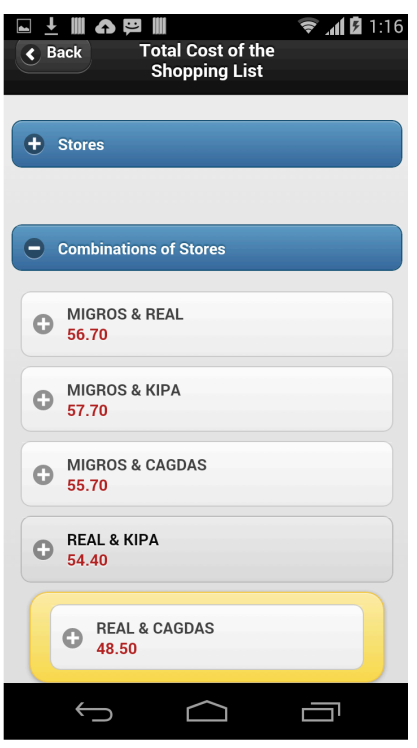

(c)

Figure 6.6 Sample screens of the purchase recommender module

\subsection{Post-Purchase Review Module}

This module facilitates the post-purchase evaluation stage by sharing reviews of a product with other users. The user chooses a product from the purchase history, or uses barcode scanning for selecting the product to be reviewed. Then, the user fills the review form which includes a flip toggle switch to specify whether the user recommends the product or not, a five-point scale rating bar, and two textboxes for entering the review title and review text as seen in Figure 6.7(a). After submitting the review form, other users can see this review in the reviews part of the product details screen. In addition, the user can see his or her own reviews in the "My reviews" screen mentioned in Chapter 6.3.

\subsection{Post-purchase Feedback Module}

This module includes a feedback form, shown in Figure 6.7(b), to share the user's level of satisfaction or dissatisfaction with a particular product, with product manufacturers. The feedback form includes six questions such as overall satisfaction about the product, the most satisfying aspect, comparison with another product, and two textboxes to post the negative and positive feedback as free-form text. In addition to the benefits of this module for the consumer, post-purchase feedback is important for product manufacturers to determine whether a product meets consumers' needs. 


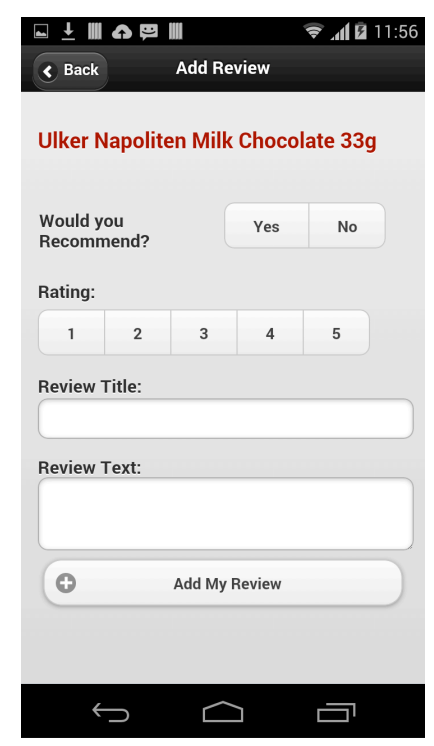

(a)

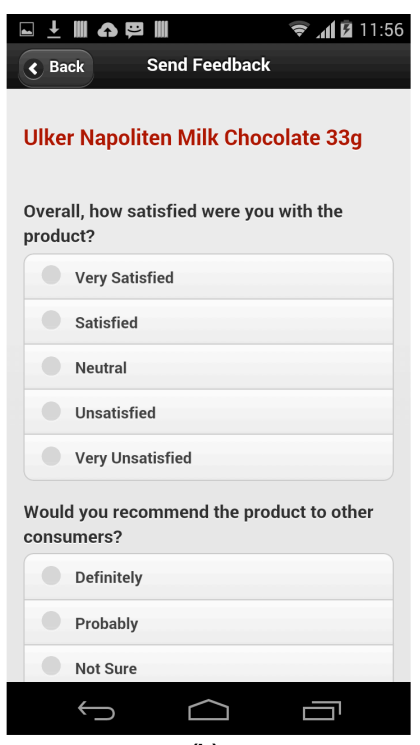

(b)

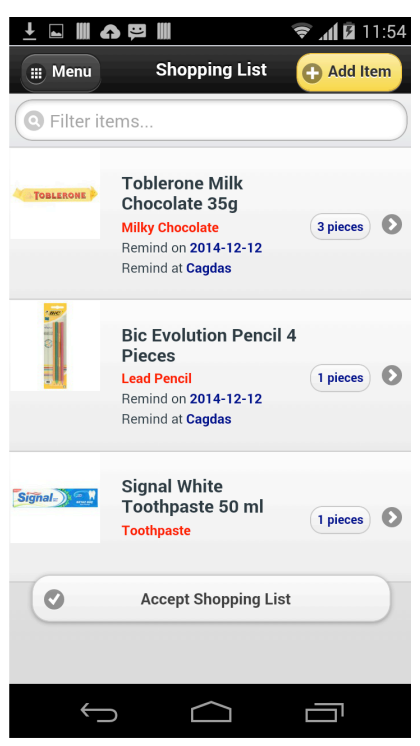

(c)

Figure 6.7 (a) Add review (b) Send feedback (c) Shopping list

\subsection{Post-purchase Consumer Support Module}

This module includes a support form to enable sending a support request about a purchased product to its manufacturer or vendor. The user selects the product for which the support is requested, as well as the manufacturer or the vendor of the product, and enters the text of the support request. The user can also take a photograph or record video of the product using the smartphone's camera, and attach it to the support form as part of the submission for describing the problem effectively.

\subsection{Shopping List Module}

Using this module, the user manages the shopping list, which is a repository for the products needed by the user as shown in Figure 6.7(c). The user adds a new product type to the shopping list by completing the "add item" form that consists of a select box including a hierarchical list of the product taxonomy, item quantity, and promotion and reminder options.

This module helps recall products needed, and provides functions used by other modules. For example, it provides reminder and promotion options for the contextaware reminder and context-aware personal promotion modules, while adding a new item to the shopping list. In addition, the user selects a product type in the shopping list to specify a particular product using the multi-criteria product recommender module. Also, the purchase recommendation module calculates the total cost using 
the items in the shopping list to determine the minimum cost at a store or at store combinations.

\subsection{Data Collector Module}

The main goal of the data collector module is to provide the data needed by the other modules. For this purpose, it delivers the collected data by the other modules to the database, and requests the required data by the other modules from the database. For example, this module sends a user's review, which is created by the post-purchase review module to the database, and requests user reviews from the database for the review search module.

This module also collects the user input. Therefore, the barcode scanning function is implemented within this module as a user input method to identify products. For example, a user can easily search for a product via barcode scanning using the mobile phone's camera, and see the product information in the "product details" screen of the information aggregator module. 


\section{CHAPTER 7}

\section{DESIGN EVALUATION}

\subsection{Usability Evaluation}

In this research, a usability test is designed to evaluate the effect of the developed prototype on user performance and user experience, as well as to assess if it is effective, efficient and satisfactory, and if it has any superiority over conventional methods used in the consumer decision process. The MobileCDP framework consists of a wide range of features. Accordingly, this usability study does not cover all features of the components of MobileCDP; it focuses on decision support functionalities of the prototype.

A mixed data collection method combining quantitative performance measures (the task completion time and error rate) with qualitative measures (the usability questionnaire) is used.

\subsubsection{Method}

International Organization for Standardization (ISO) defines the usability of a product as "the extent to which the product can be used by specified users to achieve specified goals with effectiveness, efficiency, and satisfaction in a specified context of use" [152]. System Usability Scale (SUS) was selected as the usability questionnaire for the qualitative usability evaluation [153]. The SUS consists of 10 five-point Likert scale type items that are alternating five positive statements and five negative statements to have respondents read each statement and make an effort to think whether they agree or disagree with it [153]. Bangor, et al. [154] analyzed 2324 SUS questionnaires from 206 usability tests nearly 10 year's worth, and they concluded that the SUS is a highly reliable (alpha $=0.91$ ), robust and versatile tool for usability testing.

\subsubsection{Task Design}

Six tasks are determined for experiments as shown in Table 7.1. Briefly, the first four tasks are adding products to the shopping list according to the given criteria, the fifth task is finding the minimum-cost store, and the sixth task is finding the minimum- 
cost store combination. In consumer decision process terms, the first four tasks correspond to the information search and evaluation of alternatives stages, and the fifth and the sixth tasks correspond to the evaluation of alternatives and purchase stages. First, the participants were asked to complete these tasks with conventional methods. Then, they were asked to complete the same tasks with the prototype application. The first case is named "the no-prototype case", and the second "the prototype case". Prices and features of products are differentiated for these two cases to prevent learning effects.

For the no-prototype case, the participants recorded their findings in a given form. Error rates were measured using the data in these forms. Product prices (a total of 4 product types with 4 alternatives each) for each store (a total of 4 ), and the transportation costs are given as two separate lists. Participants used these lists to complete Task 1, 2, 5, and 6. Product details such as ingredients were also given as a list for Task 3 and Task 4 . .

Table 7.1 Defined tasks for experiments

\begin{tabular}{ll}
\hline Task \# & Task Description \\
\hline Task 1 & $\begin{array}{l}\text { Suppose that you want to buy a pencil. The only criterion for a pencil is the } \\
\text { price; therefore add the cheapest pencil to your shopping list. }\end{array}$ \\
\hline Task 2 & $\begin{array}{l}\text { Suppose that you want to buy toothpaste. Again, the only criterion is the price } \\
\text { of toothpaste, but the unit price is important. For example, if } 50 \mathrm{ml} \text { toothpaste is } \\
\text { \$10, and } 100 \mathrm{ml} \text { toothpaste is } \$ 15, \text { then } 100 \mathrm{ml} \text { toothpaste is the cheaper one } \\
\text { considering the unit price. Add the cheapest one to your shopping list } \\
\text { considering the unit price. }\end{array}$ \\
\hline Task 3 & $\begin{array}{l}\text { Suppose that you want to buy a milk chocolate. However, you have gluten } \\
\text { allergy, and you do not want to buy a chocolate with gluten in it. In addition, you } \\
\text { do not want to buy a chocolate that contains additives such as E407, E551, } \\
\text { and sweeteners such as glucose syrup and fructose syrup. Add the chocolate } \\
\text { that does not contain the mentioned allergen (gluten), additives and } \\
\text { sweeteners. }\end{array}$ \\
\hline Task 4 & $\begin{array}{l}\text { Suppose that you want to buy a shampoo that does not contain toxic } \\
\text { ingredients that are identified as Sodium Lauryl Sulfate and Sodium Laureth } \\
\text { Sulfate. Add the shampoo that does not contain these chemicals. }\end{array}$ \\
\hline Task 5 & $\begin{array}{l}\text { Find the store that has the minimum cost. The cost of a store is the sum of the } \\
\text { prices of products in your shopping list, and the transportation cost to that } \\
\text { store. }\end{array}$ \\
\hline Task 6 & $\begin{array}{l}\text { Find the store combination (two stores) that has the minimum cost. The cost of } \\
\text { a store combination is the sum of the minimum prices of products in your } \\
\text { shopping list, and the transportation cost to the two stores. }\end{array}$ \\
\hline
\end{tabular}


For the prototype case, a mobile phone that runs Android OS (Motorola Moto G) and the developed prototype application were used. User interactions with the prototype, and timestamps of these interactions were logged automatically by the prototype application.

\subsubsection{Experimental Design}

Before the evaluation took place, a pilot study was conducted with five participants, in order to refine the methodology and tasks. The whole test, including performing tasks and completing the questionnaire took a maximum of 32 minutes ( $M=24.4$ $S D=4.2$ ) for the pilot study. Two participants found two of the tasks ambiguous, and therefore the wording of these tasks was modified after the pilot study.

The tests were conducted in the participants' offices for reasons of comfort. Before the tests, participants were informed about the study, test procedures, tasks, questionnaire, and estimated duration. The participants were provided orally, as well as written instructions. During the tests, the participants were free to leave a task any time. However, none of the participants left any task incomplete. The participants were free to use a pencil, paper, and a calculator to complete the tasks in the no-prototype case. For example, most of the participants drew a table on a sheet of paper, and used a calculator to sum up the costs to determine the minimum-cost store in Task 5.

After the test, the subjects were asked to fill out the System Usability Scale (SUS) questionnaire [153], and a background questionnaire that included questions to determine age, gender, computer, and mobile application usage frequency. The SUS questionnaire is a standard test commonly used to evaluate the usability of systems as explained in Chapter 7.1.1.

\subsubsection{Participants and Backgrounds}

According to the findings of the literature on usability evaluation, at least 20 participants were invited for the experiments. Nielsen and Landauer [155] claim that only five users are enough to identify between $50 \%$ and $85 \%$ of the problems. Tullis and Stetson [156] show that 12 users are enough to obtain a measure of the perceived usability of a system with the SUS questionnaire. According to Faulkner [157], at least $95 \%$ of the usability problems are found with 20 users.

24 volunteers participated in the study (12 males, 12 females), aged between 26 and 35 (17 between 26 and 30, 7 between 31 and 35) years old. All participants were smartphone users. On a four-point scale (daily, a few times per week, a few times per month, never), all participants indicated that they use computers and mobile applications daily. 


\subsubsection{Results}

This section presents the results of the SUS questionnaire, the comparison of the task completion times, and selection errors. A statistical significance level of 0.05 is used throughout this section.

Results of the SUS questionnaire:

The average SUS score for all participants was 90.52, and the standard deviation was 4.76. Figure 7.1 shows the distribution of the SUS scores. According to Bangor, et al. [158], systems which score between 85.5 and 90.9 on the SUS scale can be classified as having "excellent" usability. Sauro [159] proposes a grading scale based on percentile rankings of more than 5000 SUS observations. According to Sauro's scale, an average SUS score over 80.3 is grade A. These studies show that the prototype has an excellent usability and A grade SUS score.

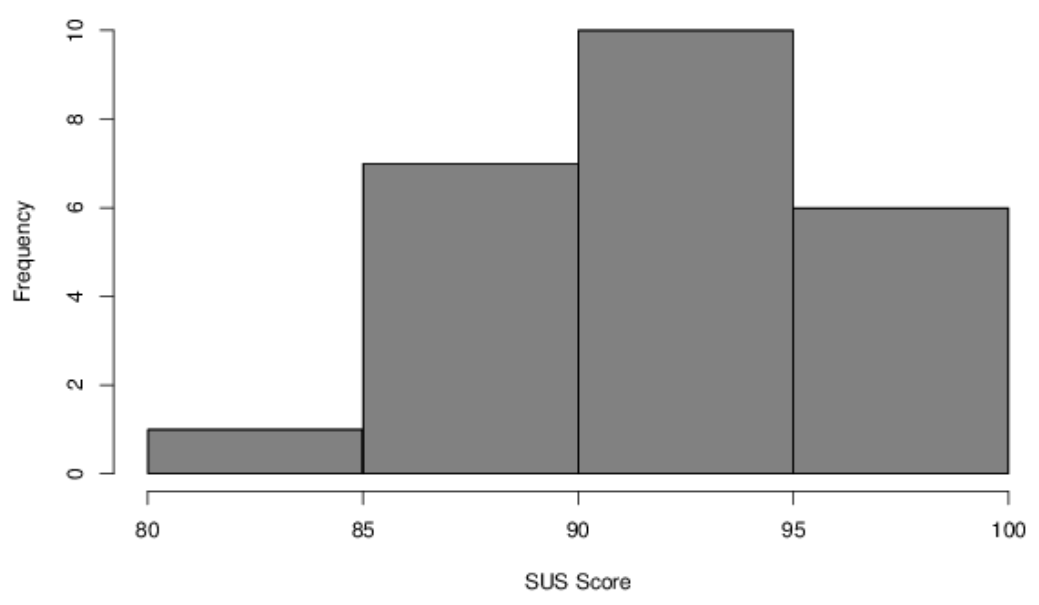

Figure 7.1 Histogram of the SUS scores of the participants

Figure 7.2 and Figure 7.3 show the results of the SUS questionnaires. As shown in Figure 7.2 , the participants gave an average rating of 4.42 or above on options related to positive statements. The participants stated that they would like to use the prototype frequently $(M=4.42, S D=0.66)$. They found the various functions in the prototype were well integrated $(M=4.58, S D=0.51)$. These results imply that the integrated approach of MobileCDP is validated. The participants also state that they felt very confident using the system $(M=4.46, S D=0.51)$. Moreover, they thought the system was easy to use $(M=4.79, S D=0.42)$, and easy to learn $(M=4.67, S D=0.49)$. As Figure 7.3 shows, the participants gave an average rating of 1.58 or below on the options related to negative statements. Overall, the questionnaire results indicated that users found the features of the prototype useful, and ready to use. 


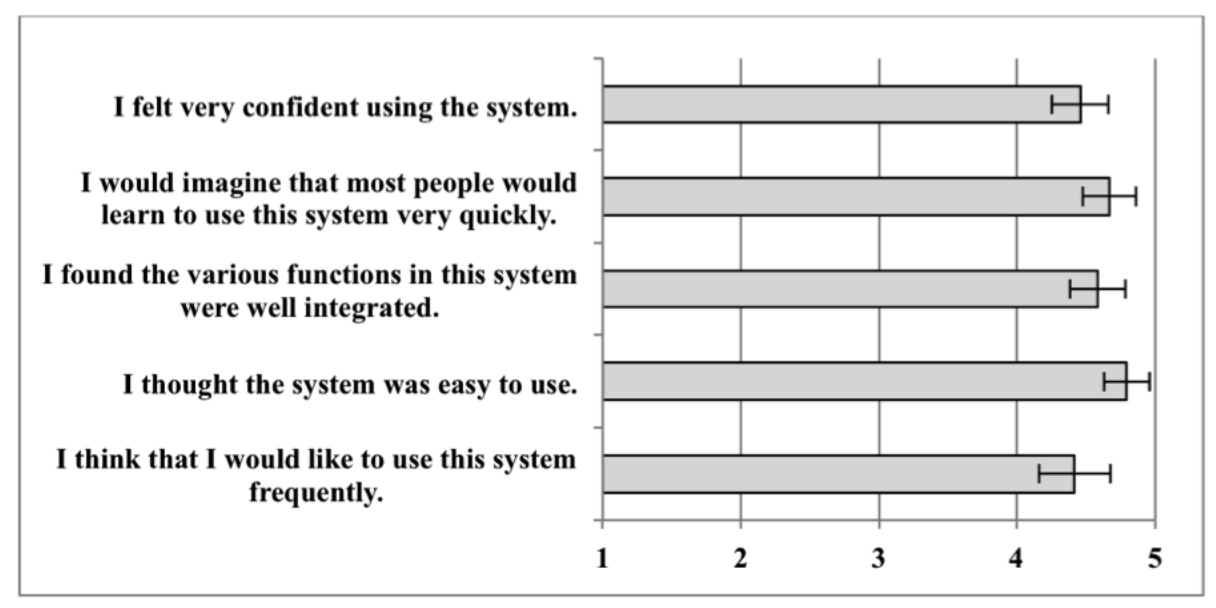

Figure 7.2 Result-set 1 from the SUS questionnaire (averages, higher rating is better, and error bars indicate standard deviations)

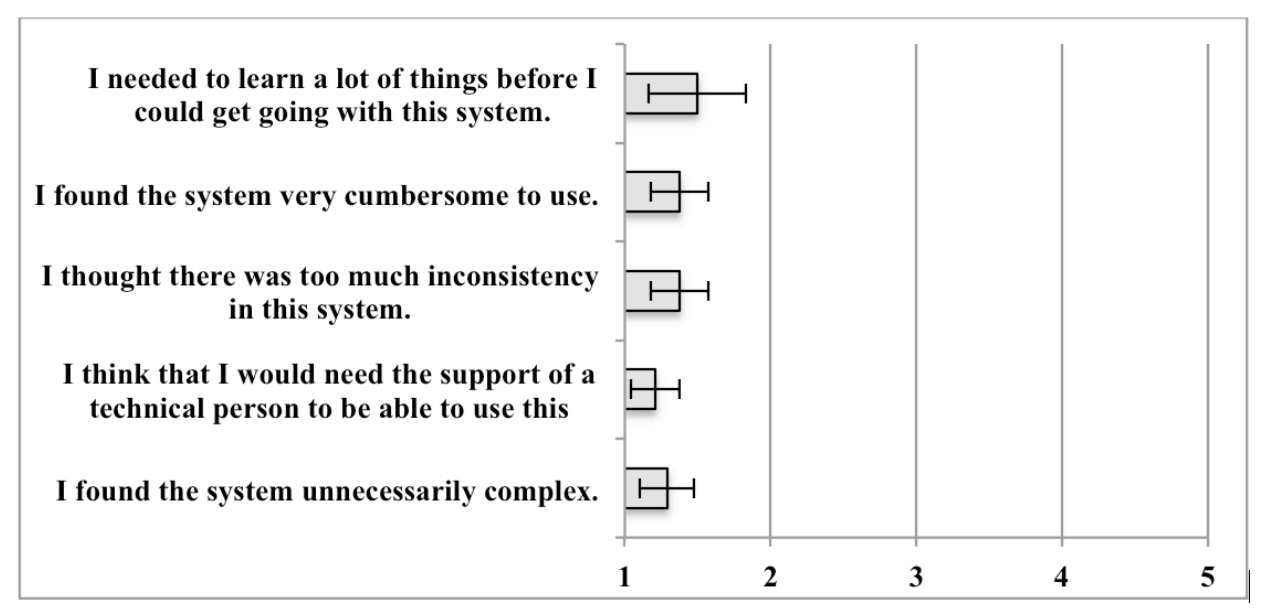

Figure 7.3 Result-set 2 from the SUS questionnaire (averages, lower rating is better, and error bars indicate standard deviations)

\section{Comparison of task completion times:}

Average task completion times (TCTs) for all six tasks are given in Figure 7.4. A paired-samples t-test was conducted to compare TCTs in the prototype and the noprototype cases in Figure 7.4. As shown in Table 7.2, there is a significant difference for all six tasks $(p<0.001)$ in the TCTs for the prototype and no-prototype cases. These results imply that the prototype is timesaving. Moreover, decreased TCTs imply that the prototype reduces the cognitive effort required by the consumer to complete the tasks. 
In addition to comparing TCTs for each task using the paired-samples t-test, a two way, repeated measures ANOVA test was also conducted to compare the main effect of the task type (the no-prototype and the prototype) on TCTs. The ANOVA test results also indicate that the task type has a significant effect on the time spent to complete the tasks $(F(1,23)=678.709, p<0.001)$. As a limitation, the participants perform the tasks in the same order. They performed the no-prototype tasks, and the prototype tasks, respectively.

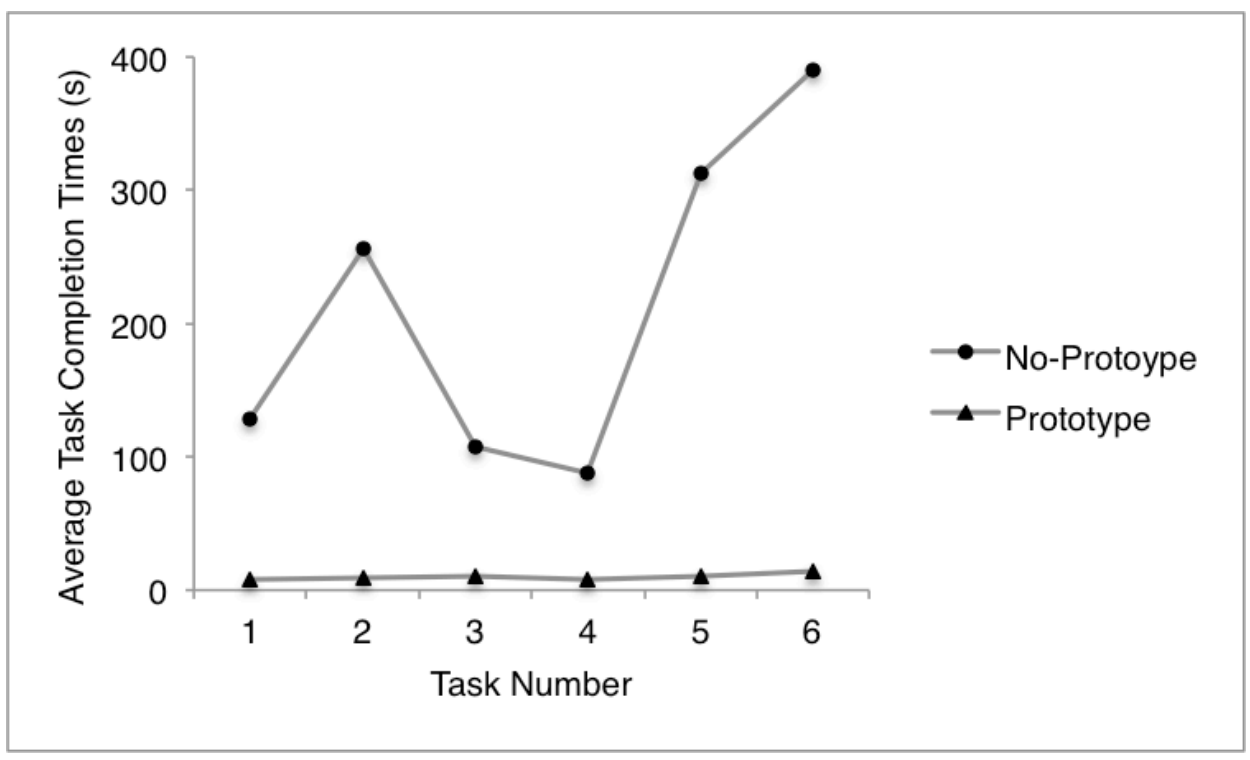

Figure 7.4 Average Task Completion Time (TCT) for each task in the no-prototype and the prototype cases.

Table 7.2 Descriptive statistics and t-test results (seconds)

\begin{tabular}{|c|c|c|c|c|c|c|c|c|}
\hline \multirow[b]{2}{*}{ Task } & \multicolumn{2}{|c|}{ No-Prototype } & \multicolumn{2}{|c|}{ Prototype } & \multirow[b]{2}{*}{$\mathbf{N}$} & \multirow[b]{2}{*}{$\mathbf{t}$} & \multirow[b]{2}{*}{ df } & \multirow[b]{2}{*}{ Sig. } \\
\hline & Mean & Std.Dev & Mean & Std.Dev & & & & \\
\hline Task 1 & 128.58 & 41.026 & 7.75 & 3.220 & 24 & 14.289 & 23 & $<.001$ \\
\hline Task 2 & 256.71 & 91.851 & 8.75 & 2.691 & 24 & 13.334 & 23 & $<.001$ \\
\hline Task 3 & 107.92 & 39.989 & 10.21 & 3.257 & 24 & 12.070 & 23 & $<.001$ \\
\hline Task 4 & 87.58 & 27.639 & 8.04 & 2.493 & 24 & 13.739 & 23 & $<.001$ \\
\hline Task 5 & 313.38 & 92.998 & 10.50 & 1.842 & 24 & 15.996 & 23 & $<.001$ \\
\hline Task 6 & 390.63 & 75.585 & 14.50 & 2.571 & 24 & 24.197 & 23 & $<.001$ \\
\hline
\end{tabular}


Comparison of selection errors:

A selection error refers to a situation where a user chooses a wrong product for Tasks $1-4$, or a wrong store, or store combination for Task 5 and Task 6 . The numbers of selection errors were investigated to measure the effect of the prototype on the product and store selection. As shown in Table 7.3, there is no selection error in the prototype case, since the prototype makes calculations for Tasks 1, 2, 5, and 6 , and helps filter product alternatives for Task 3 and Task 4. For the no-prototype case, $33 \%$ of the participants selected a wrong store combination for Task 6 , and $21 \%$ selected a wrong store for Task 5 . Of the participants, $17 \%$ selected a wrong product for Task 2 , and $4 \%$ of the participants selected wrong products for each of Task1, Task3, and Task4.

These results show that participants could not select the minimum cost products and stores in the no-prototype case, while they selected the correct products and stores in the prototype case; which imply that the prototype is cost saving.

Table 7.3 Percentage of number of unsuccessful tasks due to selection errors

\begin{tabular}{lcccccc}
\hline & Task 1 & Task 2 & Task 3 & Task 4 & Task 5 & Task 6 \\
\hline No-prototype & 4.16 & 16.66 & 4.16 & 4.16 & 20.83 & 33.33 \\
\hline Prototype & 0 & 0 & 0 & 0 & 0 & 0 \\
\hline
\end{tabular}

\subsubsection{Summary of the Usability Evaluation}

Overall, the results of the SUS questionnaire indicate that the developed modules of the prototype are functional, useful, well integrated, and easy to use, which implies that the framework can be successfully utilized as an integrated mobile information system. Statistical comparisons of TCTs of the prototype and the no-prototype cases reveal that there is a significant difference in the TCTs of these cases $(p<0.001)$, which proves the prototype significantly decreases the time spent in the decision process, and reduces cognitive effort of users. Moreover, the comparison of the selection errors reveals that users make wrong decisions when selecting minimum cost products and stores without the prototype; hence, the prototype also decreases the cost.

\subsection{Accuracy Evaluation of the Proposed Data Collection Methods}

In addition to experiments on the prototype, we also evaluate the proposed data collection methods explained in Chapter 5.2. In this section, accuracy evaluation of 
the proposed methods is presented. First, the performance metrics for the evaluation are defined. Then, experiments on the proposed image-based and videobased methods are presented respectively.

\subsubsection{Performance Metrics}

Accurate recognition is an important feature of an OCR system. In order to measure the correct recognition performance of the proposed method, the following metrics are defined:

\section{Metric 1 - Character Recognition Rate (CRR):}

The character recognition rate is the ratio of the number of correctly recognized characters to the number of all characters in the ground truth.

$$
C R R=\frac{N_{C}}{N}, N_{c}=N-L \quad \text { (Equation 7.1) }
$$

$N$ : the total number of the characters in the ground truth

$N_{C}$ : the number of correctly recognized characters

$L$ : the sum of the Levenshtein distances between the words in the ground truth and the corresponding words in the recognized text.

Metric 2 - Word Recognition Rate (WRR)

According to this metric, the inaccurate recognition of one or more characters of a word means that the word is recognized inaccurately. Therefore, if the Levenshtein distance between a word in the ground truth and the corresponding word in the recognized text is not equal to "0", it means that the word in the recognized text is inaccurate. The word recognition rate is the ratio of the number of correctly recognized words to the number of all words in the ground truth.

$$
W R R=\frac{N_{W}}{N}, N_{W}=N-L \quad(\text { Equation 7.2) }
$$

$N$ : the total number of the words in the ground truth

$N_{W}:$ the number of correctly recognized words

$L:$ the number of inaccurately recognized words 


\subsubsection{Experiments for the Image-Based Data Collection Method}

In these experiments, 45 store receipts from 3 different grocery stores are used. These store receipts have the same font type and 800 characters on average. 20 of the store receipts are used to train the OCR engine, and the other 25 store receipts are used to measure the accuracy of the proposed method. The store receipt images are captured by a mobile phone at $2448 \times 3264$ pixels ( 5 megapixels).

A sample store receipt image is given in Figure 7.5. As shown in the figure, the store receipt images that are used in this study exhibit some problems such as text skew, insufficient lighting, shadows, and grains.

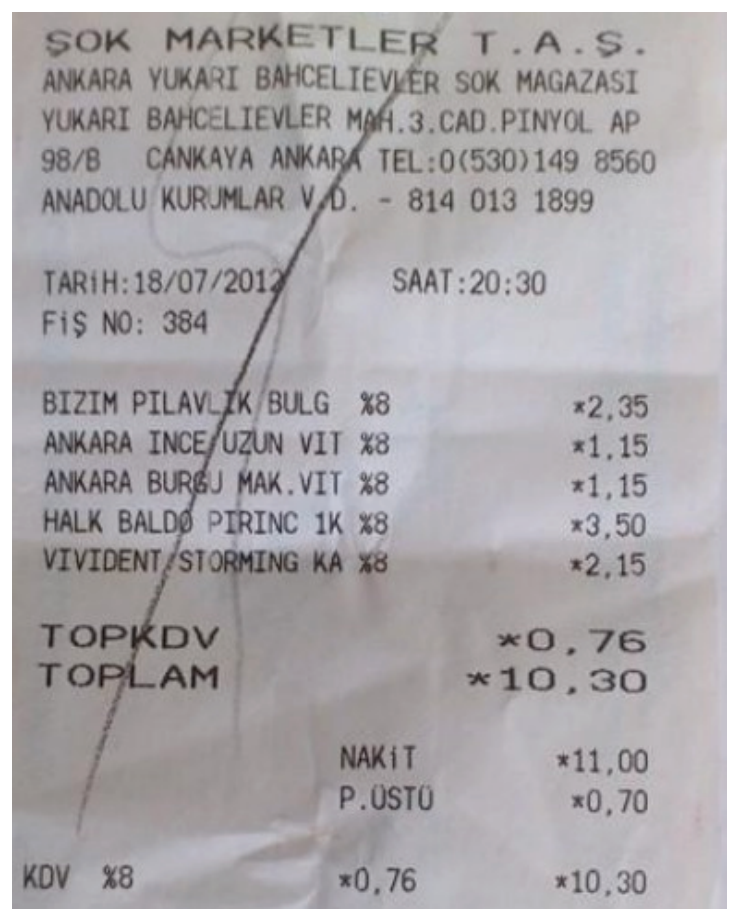

Figure 7.5 A sample receipt captured by the mobile phone camera

As mentioned above, the word and character accuracy rates are selected as performance metrics in the experiments conducted within the scope of this study. The word and character recognition performances of the processes applied on the store receipt images are given in Table 7.4.

First, experiments for the image-based scheme are conducted. During these experiments, the Tesseract OCR engine is used with its default language files (Turkish and English). In these experiments, recognition performances appear to be low: $41 \%$ for word recognition, and $45 \%$ for character recognition, since the fonts in the store receipts are not commonly used, and so are not included in the default language files of the OCR engine. 
Table 7.4 Word and character recognition rates of the image based method

\begin{tabular}{lcc}
\hline \multicolumn{1}{c}{ Process } & \multicolumn{1}{c}{$\begin{array}{c}\text { Word } \\
\text { Recognition Rate }\end{array}$} & $\begin{array}{c}\text { Character } \\
\text { Recognition Rate }\end{array}$ \\
\hline $\begin{array}{l}\text { Untrained Optical Character } \\
\text { Recognition }\end{array}$ & $33 \%$ & $35 \%$ \\
\hline $\begin{array}{l}\text { Trained Optical Character } \\
\text { Recognition }\end{array}$ & $49 \%$ & $53 \%$ \\
\hline Knowledge Based Correction & $69 \%$ & $73 \%$ \\
\hline
\end{tabular}

Afterwards, the OCR engine is trained with a training set that includes 20 receipts and more than 16000 characters in order to improve the recognition performance. As a result of the experiments performed with the trained OCR engine, the word recognition performance is increased from $33 \%$ to $49 \%$, and the character recognition performance is improved from $35 \%$ to $53 \%$.

Applying the KBC algorithm after the OCR process improves the word recognition performance to $69 \%$, and the character recognition performance to $73 \%$.

These experiments show that the training process improves the recognition rates. Moreover, the proposed KBC method significantly increases the word and character recognition rates, even much more than the training.

\subsubsection{Experiments for the Video-Based Data Collection Method}

In this section, we report our results based on the experiments performed with 20 store receipts. Each receipt includes approximately 500 characters and 90 words on average, and its video is captured by a mobile phone at $640 \times 480$ pixel resolution with H.264/MPEG4-AVC encoding. A representative frame of the captured videos is given in Figure 7.6. As shown in the figure, the store receipts in the videos exhibit some problems such as text skew, insufficient lighting and grains.

Prior to the experiments, the Tesseract OCR engine is trained with a different set of 20 store receipts having the same font type. 


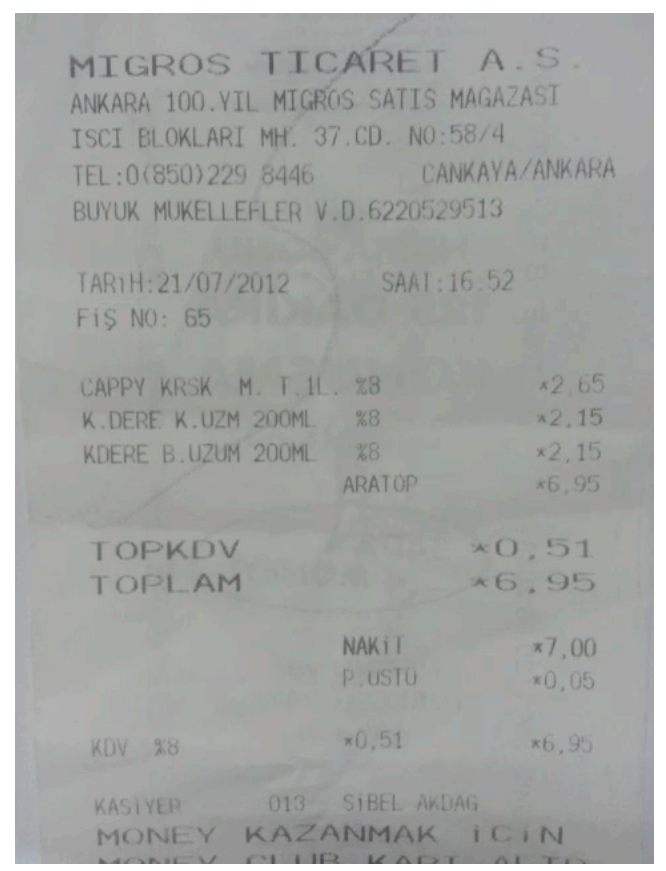

Figure 7.6 A sample frame of a captured video

Experiments on the accuracy performance:

A number of experiments are conducted to measure the success of our proposed Text Clustering Based Multiple Frame Integration (TB-MFI) approach. TB-MFI and super resolution (SR) methods use consecutive frames in a video sequence of a store receipt. The same number of consecutive frames is used in TB-MFI and SR experiments in order to have a fair comparison. 12 frames of each video sequence are used for the experiments, as explained in Sec. 4.3.

In these experiments, accuracy performances for a single frame, the super resolution method, and the proposed method are compared.

The results of single frame:

In this experiment, a single frame of the captured video sequence is used. For each video sequence, the frame that has the highest word recognition rate in the video is selected for comparison. For example, if the fifth extracted frame has the highest word recognition rate in a video sequence, the fifth frame is selected for comparison for that sequence. According to the results of these experiments, the average word recognition rate is $63.7 \%$ and the character recognition rate is $73.9 \%$. 
The results of super resolution:

As mentioned in Chapter 2.2, the $L_{1}$ norm minimization and robust regularization method given in [100] is selected as the super resolution (SR) method for comparison with our proposed method, since the authors state that their experiments confirm the effectiveness of their method and demonstrate its superiority to other super-resolution methods. Accordingly, in our experiments, we use the Matlab software package provided by the authors, with the default settings for the SR method: regularization factor: 0.005, number of iterations: 50, regularization spatial decaying coef: 0.8 , regularization kernel size: 2 , and step size: 10. According to our experiments, the super resolution method is successful and increases the average word recognition rate from $63.7 \%$ to $74.8 \%$, and the average character recognition rate from $73.9 \%$ to $86.0 \%$, as compared with the single frame results.

Results of the proposed Text Clustering Based Multi-frame Integration (TB-MFI) method:

Figure 7.7 shows the comparison of average accurate recognition rates for the single frame, super resolution, and TB-MFI approaches. For our proposed method, TB-MFI, the average word and character recognition rates increase from 63.7 to $79.7 \%$ and $73.9 \%$ to $92.1 \%$, respectively, when compared with the single image results. These experiments show that the difference between the word and character recognition rates of the proposed method and the single frame is $16.0 \%$ and $18.2 \%$, respectively.

A comparison of these results indicates that both the super resolution method and the proposed method noticeably improves recognition rates, however, the proposed method provides more improvement than the super resolution method.

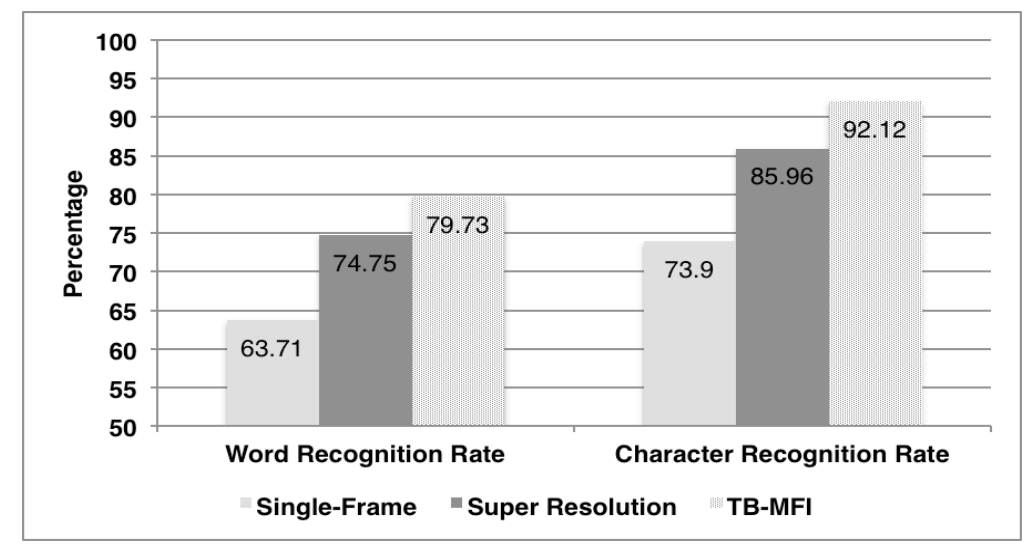

Figure 7.7 Comparison of the proposed TB-MFI method with Super Resolution and Single Frame 
Table 7.5 presents the results for 20 video sequences individually. Since these videos are captured by a hand-held mobile phone, each video sequence is unique. However, as can be seen in Table 7.5, the word and character accuracy results appear to be consistent for different video sequences, and there are no significant differences between the maximum and minimum recognition rates for each method.

Table 7.5 Word and character recognition rates of individual video sequences

\begin{tabular}{ccccccc}
\hline & \multicolumn{2}{c}{ Word Recognition Rate } & \multicolumn{2}{c}{ Character Recognition Rate } \\
\hline \multirow{2}{*}{ ID } & Single & $\begin{array}{c}\text { Super } \\
\text { Resolution }\end{array}$ & $\begin{array}{c}\text { TB- } \\
\text { MFI }\end{array}$ & $\begin{array}{c}\text { Single } \\
\text { Frame }\end{array}$ & $\begin{array}{c}\text { Super } \\
\text { Resolution }\end{array}$ & TB-MFI \\
\hline $\mathbf{1}$ & 58.6 & 70.8 & 75.9 & 65.7 & 83.3 & 87.2 \\
\hline $\mathbf{2}$ & 61.5 & 74.1 & 81.1 & 69.0 & 88.3 & 94.1 \\
\hline $\mathbf{3}$ & 62.2 & 73.0 & 79.0 & 72.7 & 85.7 & 90.5 \\
\hline $\mathbf{4}$ & 62.9 & 74.0 & 76.9 & 73.7 & 85.2 & 90.1 \\
\hline $\mathbf{5}$ & 63.8 & 74.2 & 77.5 & 72.3 & 86.7 & 92.9 \\
\hline $\mathbf{6}$ & 64.4 & 73.0 & 78.3 & 74.5 & 84.5 & 91.3 \\
\hline $\mathbf{7}$ & 64.0 & 74.7 & 80.0 & 76.4 & 86.0 & 89.2 \\
\hline $\mathbf{8}$ & 64.2 & 76.4 & 80.6 & 76.5 & 84.1 & 94.3 \\
\hline $\mathbf{9}$ & 67.5 & 78.9 & 83.5 & 76.6 & 88.6 & 95.9 \\
\hline $\mathbf{1 0}$ & 63.4 & 73.4 & 77.8 & 73.0 & 82.2 & 90.2 \\
\hline $\mathbf{1 1}$ & 68.8 & 80.7 & 83.5 & 82.1 & 92.7 & 95.9 \\
\hline $\mathbf{1 2}$ & 63.1 & 73.6 & 77.6 & 72.1 & 82.5 & 90.7 \\
\hline $\mathbf{1 3}$ & 59.8 & 68.8 & 76.3 & 70.3 & 79.1 & 88.3 \\
\hline $\mathbf{1 4}$ & 65.8 & 78.2 & 85.3 & 79.8 & 87.2 & 96.7 \\
\hline $\mathbf{1 5}$ & 64.2 & 74.1 & 81.8 & 72.3 & 84.5 & 95.8 \\
\hline $\mathbf{1 6}$ & 65.8 & 77.6 & 80.3 & 77.2 & 89.4 & 93.3 \\
\hline $\mathbf{1 7}$ & 63.4 & 75.6 & 79.6 & 71.6 & 88.7 & 91.0 \\
\hline $\mathbf{1 8}$ & 62.3 & 75.6 & 80.8 & 73.1 & 86.4 & 89.7 \\
\hline $\mathbf{1 9}$ & 63.8 & 73.1 & 78.3 & 74.1 & 86.3 & 91.1 \\
\hline $\mathbf{2 0}$ & 64.7 & 75.1 & 80.6 & 75.1 & 87.8 & 94.2 \\
\hline $\mathbf{A v e r a g e}$ & 63.7 & 74.8 & 79.7 & 73.9 & 86.0 & 92.1 \\
\hline & & & & & & \\
\hline & & & & & & \\
\hline
\end{tabular}


The results of the experiments on the number of frames:

In these experiments, we measure how the number of frames affects the word and character recognition rates. As shown in Figure 7.8, word and character recognition rates increase until 12 frames and then start to decrease for larger numbers of frames, for the super resolution method. We believe that the propagation of errors within the motion estimation and reconstruction procedures results in a degradation in the obtained high resolution (HR) image, and this degradation increases errors in the character recognition procedure. On the other hand, for the proposed method, if the number of frames used increases, word and character recognition rates also increase, or remain stable. Most of the inaccurate recognition errors are eliminated in the cluster elimination and final text generation steps in the proposed method. These results show that the proposed method is more robust than the super resolution method.

Since the accuracy performance reduces after 12 frames for the super resolution method, 12 frames of each video sequence are used for all the experiments. The SR method needs a minimum of 4 frames to increase the resolution from $640 \times 480$ to $1280 \times 960$.

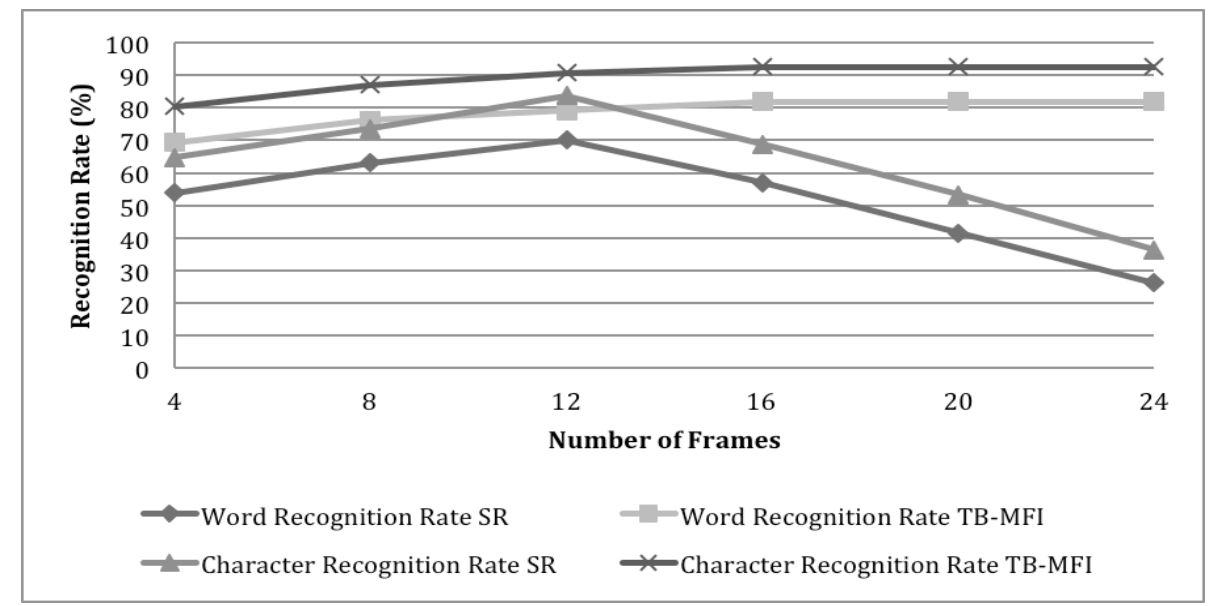

Figure 7.8 Word and character recognition rates versus the number of frames

Summary of the experiments for the video-based method:

In this section, the recognition accuracies in the case of a single image, the proposed method, and the super resolution method are compared by using real video captured by handheld mobile phones. The experiments show that the proposed method improves the word recognition rate from $63.7 \%$ to $79.7 \%$ and the character recognition rate from $73.9 \%$ to $92.1 \%$, on average, when compared with the single image results. Experiments indicate that the super resolution method also improves the word recognition rate from $63.7 \%$ to $74.8 \%$ and the character 
recognition rate from $73.9 \%$ to $86.0 \%$. However, the proposed method achieves even more improvement when compared to the super resolution method.

Another major finding is that the proposed method is more robust than the super resolution method. It is interesting to note that if more than 12 frames are used for the super resolution method, the word and character recognition rates decrease significantly. This result may be explained by the fact that propagation of the errors within the motion estimation and reconstruction procedures may degrade the reconstructed high-resolution image. Hovewer, it is also shown that there is a positive correlation between the number of used frames and improvements in the recognition rates for the proposed method. As the number of frames increases, the number of text lines used by the proposed algorithm increases, thereby raising the possibility of finding and combining more accurate text lines.

As a conclusion, the proposed method is successful as it is able to noticeably improve the correct recognition rates both for characters and words in the document videos captured by mobile devices. Results also show that the proposed method is more effective and robust than the compared super resolution method.

\subsection{Performance Evaluation of the Image-Based Data Collection Method}

After the development of the image-based automated data collection component, we compare the in-phone and the remote server processing approaches for mobile document image analysis in order to explore their trade-offs. For the in-phone approach, all processes required for mobile document image analysis run on the mobile phone. On the other hand, in the remote-server approach, the core OCR process runs on the remote server and other processes run on the mobile phone.

In this section, first of all, we present our methodology to compare speed and accuracy performances of the in-phone and the remote server approaches.

\subsubsection{Phases of the Converting a Captured Image into Text}

The phases presented below are followed in order to convert a mobile phone captured image into electronic text:

- Initialization phase: This phase includes the initialization of the Tesseract TessBaseAPI, which is a Java interface for the Tesseract OCR. Both the server and the phone applications use the same API.

- Compression phase: This phase consists of writing a compressed version of the source bitmap to a specified output stream. Bitmap.compress method of the Android API is used to compress the source bitmap. In case the compression setting is "off", this phase is bypassed. 
- Decoding phase: This phase includes decoding the output stream of the compression phase into a bitmap. BitmapFactory.decodeStream method of the Android API is used for the decode phase. If the compression setting is "off", this phase is skipped.

- Image sending phase: In this phase, the bitmap file is sent from the client (phone) side to the remote server. The in-phone approach does not include this phase.

- Image setting phase: This phase includes setting the bitmap file for the OCR operation. The input of this phase is the output bitmap of the previous phase.

- OCR phase: The OCR operation is performed in this phase. The input of this phase is the image set in the previous phase. The output is the recognized text by the Tesseract OCR engine.

- Text sending phase: In this phase, the recognized text is sent from the remote server to the client (phone) side. Only remote-server approach includes this phase.

\subsubsection{Measuring the Speed Performance}

The image-based method can be applied on a mobile phone as well as a remote server. Then, we compare speed performances of the in-phone and the remote server approaches for mobile document image analysis. We define the following performance metrics based on the phases defined above.

- Total Execution Time: It is the time period between the beginning of the initialization phase and the end of the OCR phase. For the in-phone approach, total execution time includes the initialization, image setting and OCR phases. For the remote server approach, total execution time also includes compression, decoding, image sending and text sending phases.

- OCR Time: It includes recognition of the text from the image set in the image setting phase.

- Network Delay: It is the required time to complete the image and text sending phases. Image is sent from the mobile phone to the remote server to make the OCR operation in the server, and the extracted text is sent from the remote server to the mobile phone to display as the recognized text. The value of this performance metric is always " 0 " for the in-phone approach, since this approach does not include any network transmission.

- Compression and Decode Delay: It is the time period between the beginning of the compression phase and the end of the decoding phase. Therefore, this period includes the compression of the bitmap to an output stream and decoding the compressed output stream into a bitmap. The value of this 
performance metric is always "0" for tests performed with uncompressed images, since these tests do not include compression and decoding phases.

Initialization and image setting phases are not defined as stand-alone performance metrics since they are uniform for both the in-phone and remote-server approaches, and proportions of their values in total execution time are negligible compared with the determined performance metrics. The average initialization delay is 146 milliseconds and the average image setting delay is 70 milliseconds.

\subsubsection{Parameters}

Speed and correct recognition performances of the OCR approach are dependent on the following parameters:

- Resolution: The resolution parameter is the pixel resolution of the input image for the OCR phase. Original images are captured as $1536 \times 2048$ pixels (3.2 megapixels). Captured images are downscaled to the intended resolution before initialization of the Tesseract API. Downscaling factor can be chosen from the Android application. The downscaling factor is the number of pixels in width and height that correspond to a single pixel in the decoded bitmap. For example, if downscaling factor equals to 2, application returns an image that is $1 / 2$ of the width/height of the original, and 1/4 of the number of pixels in total. Therefore, 2-factor downscaling converts a $1536 \times 2048$ pixel resolution image to a $768 \times 1024$ pixel resolution image. 1536x2048, 768x1024 (2-factor downscaling) and 384x512 pixel (4-factor downscaling) resolutions are used for our analysis. 192x256 pixel resolution (8-factor downscaling) images are also used in the experiments, but the results of these experiments are not included in the analysis since WRR and CRR are below $\% 5$ for this resolution.

- Compression Quality (Q-factor): If the compression setting is not "off", the source bitmap is compressed according to "compression quality" setting in the Android application. We compressed source images using JPEG with different quality settings, namely Q-factor (quality factor). Q-factor can vary between 0 and 100. 0 means that compression for small size and minimum quality, while 100 means that compression for maximum quality. Q-factors of $100,75,50$ and 25 s are used during our tests.

\subsubsection{Experimental Setup}

All measurement results are obtained on the following real hardware platforms.

\section{Dataset:}

In this study, 26 store receipts are used. A receipt includes 572 characters and 95 words on average. 
The in-phone approach:

A Samsung 19300 S3 phone is used in our experiments, which has a $3264 \times 2448$ pixel autofocus camera, quad-core $1.4 \mathrm{GHz}$ Cortex-A9 CPU and $1 \mathrm{~GB}$ RAM. Android 4.1.1 Jelly Bean operating system runs on the mobile phone.

All processes are performed in the mobile phone in this approach. At first, Tesseract API is initialized. If the compression setting is not "off", previously captured image is compressed with the selected Q-factor. Then, the image is set for the OCR operation as a bitmap. After that, information on this image is extracted by using the OCR engine running on the mobile phone. Finally, the recognized text is displayed on the mobile phone.

\section{The remote server approach:}

The server includes a two-core Intel Xeon E3-1220 3.10GHz CPU and 8 GB RAM. 64-bit Ubuntu 12.04 Precise operating system runs on the remote server.

In this approach, OCR is performed in the remote server. After initialization of the Tesseract API, previously captured image is compressed with the chosen Q-factor. Next, this image is sent to the remote server. Then, the OCR engine running on the remote server extracts the text on the received image. Finally, the extracted text is sent to the mobile phone and displayed to the user.

In this setup, the remote server is accessible through a wide area network. The mobile phone is connected to the Internet via an ADSL connection with a $0.84 \mathrm{Mbps}$ upload rate and $5.30 \mathrm{Mbps}$ download rate. The remote server is connected to the Internet with 100BaseTX Ethernet connection that has a $19.01 \mathrm{Mbps}$ upload rate and 73.28 Mbps download rate. Download and upload rates are measured with speedtest.net [160] internet speed test service.

\subsubsection{Comparing Speed Performance}

For both the in-phone and the remote server approaches, we perform experiments on each possible combination of resolution and Q-factor parameters.

Figure 7.9(a) compares the total execution time (TET) of the in-phone approach according to different resolution and compression settings. It can be seen that compression increases TET, since it adds extra "compression and decode" delay as defined in Chapter 7.3.2. The 2-factor downscaling of the image slightly decreases TET (2591 $\mathrm{ms}$ for the original image and $2531 \mathrm{~ms}$ for the downscaled image). However, 4-factor downscaling significantly increases TET to $5293 \mathrm{~ms}$, since it doubles the OCR time. Overall, 2-factor downscaling without compression yields the minimum TET for the in-phone OCR (2531 ms).

Figure 7.9(b) illustrates the TET per resolution and Q-factor for the remote server approach. The first thing to note is that downscaling the image significantly 
decreases the TET. It is interesting to note that $Q$-factor 75 also significantly decreases the TET, but other Q-factors slightly change TET. 4-factor downscaling provides the lowest TET values. However, it also reduces correct recognition rates.

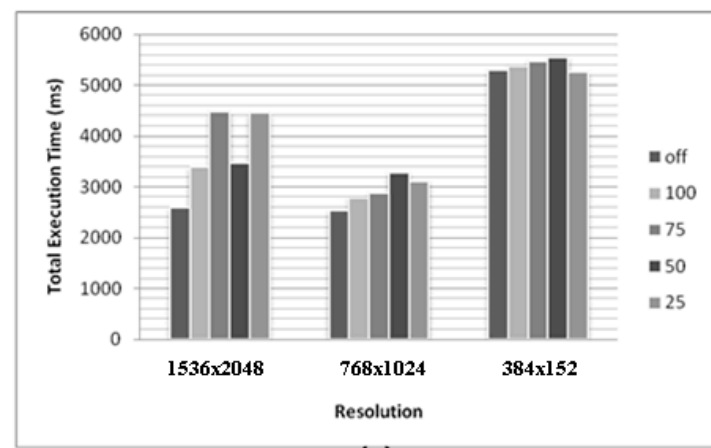

(a)

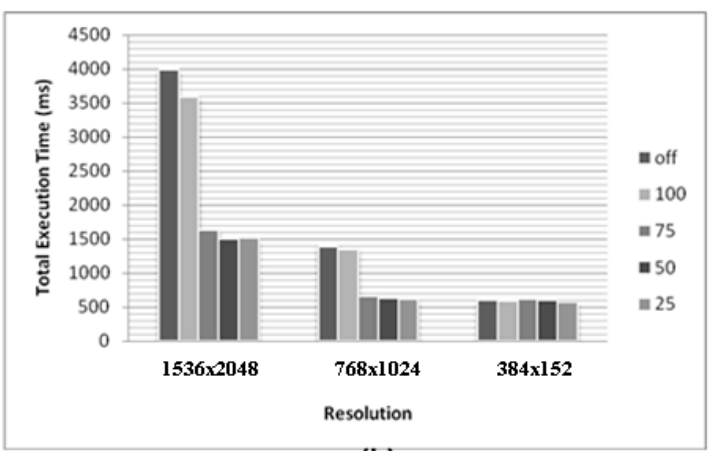

(b)

Figure 7.9 (a) Total execution time of the in-phone approach (b) Total execution time of the remote server approach

Figure 7.10(a) compares TET values of the in-phone and server approaches according to $1536 \times 2048$ resolution and varied compression settings. The in-phone approach outperforms the server approach for uncompressed images and Q-factor 100 compressed images at $1536 \times 2048$ resolution. There is a slight difference between the TET values of the in-phone and the server approaches for images compressed with $\mathrm{Q}$-factor 75 and $\mathrm{Q}$-factor 50 . On the other hand, the remote server approach surpasses the in-phone approach for Q-factor 25.

Figure $7.10(\mathrm{~b})$ shows TET values for $768 \times 1024$ resolution and varied compression settings. At this resolution, both the in-phone and the remote server approaches perform faster than $1536 \times 2048$ resolution. Similar to $1536 \times 2048$ resolution, the inphone approach outperforms the server approach for uncompressed images and Qfactor 100 compressed images, but the gap between the TET values of these approaches are decreased. The remote server approach surpasses the in-phone approach for compressed images with Q-factor 75, 50 and 25.

Figure 7.11 illustrates TET values for $384 \times 512$ resolution and different compression settings. The remote server approach outperforms the in-phone approach for all compression settings at this resolution.

Figure 7.12 illustrates the "OCR time" for each possible resolution and Q-factor combination of the in-phone and the remote server approaches. According to Figure 7.12(a), OCR time changes slightly between $1536 \times 2048$ and $768 \times 1024$ resolutions, but it is significantly increased for $384 \times 512$ resolutions for the in-phone approach. In contrast, Figure 7.12(b) shows that OCR time significantly decreases between $1536 \times 2048$ and $768 \times 1024$ resolutions, but it changes slightly for $384 \times 512$ resolutions for the remote server approach. Both the in-phone and the remote server 
approaches perform better at $768 \times 1024$ resolutions than the $1536 \times 2048$ and $384 \times 512$ resolutions.

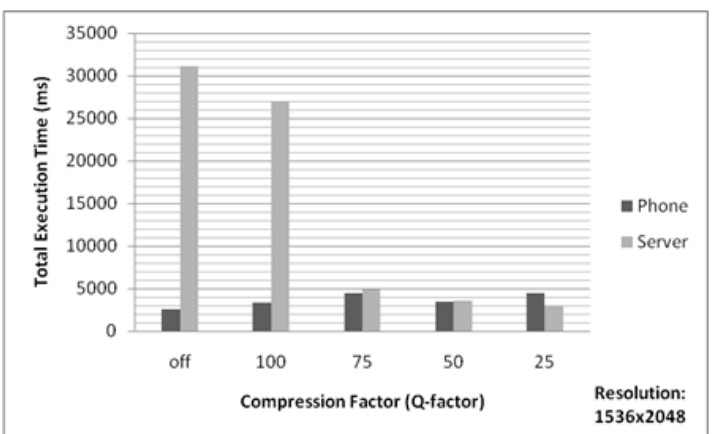

(a)

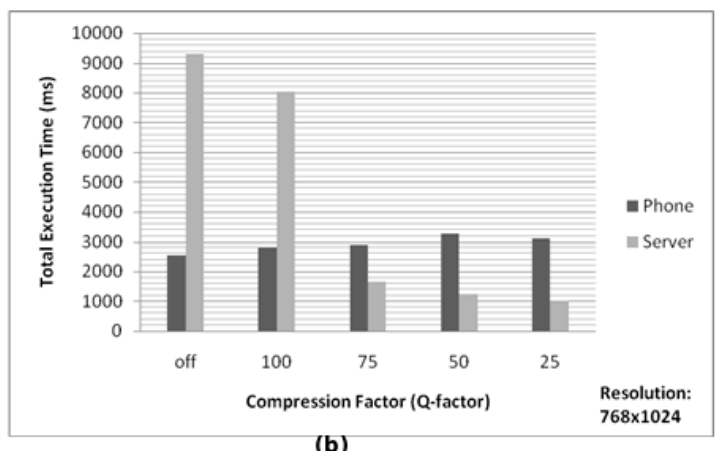

(b)

Figure 7.10 (a) Total execution time for $1536 \times 2048$ pixel resolution (b) Total execution time for $768 \times 1024$ pixel resolution

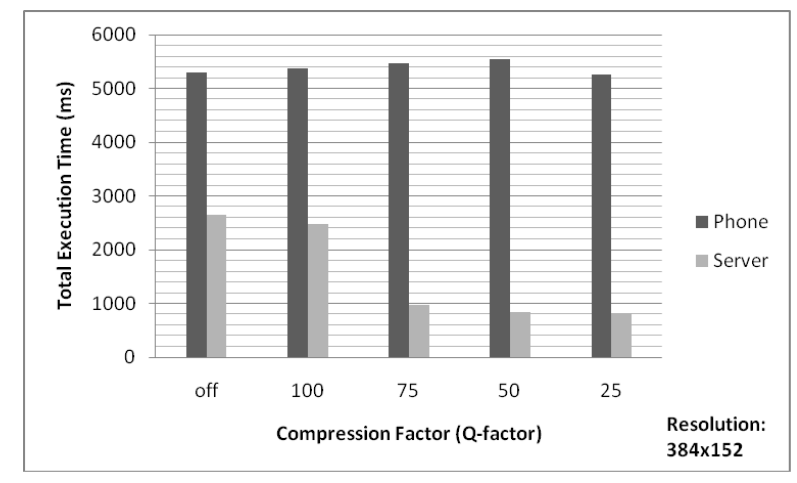

Figure 7.11 Total execution time for $384 \times 512$ pixel resolution

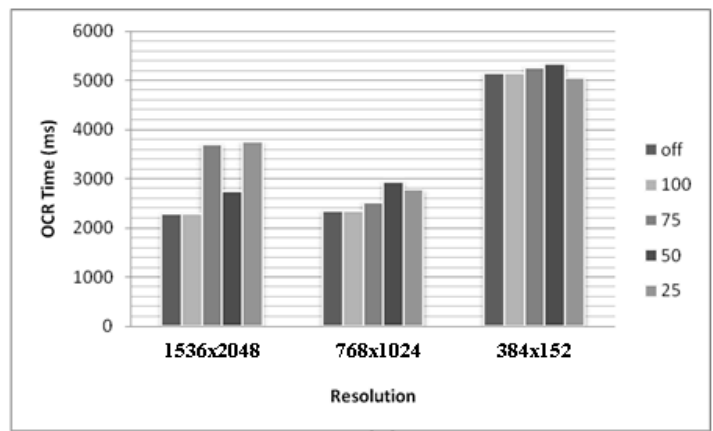

(a)

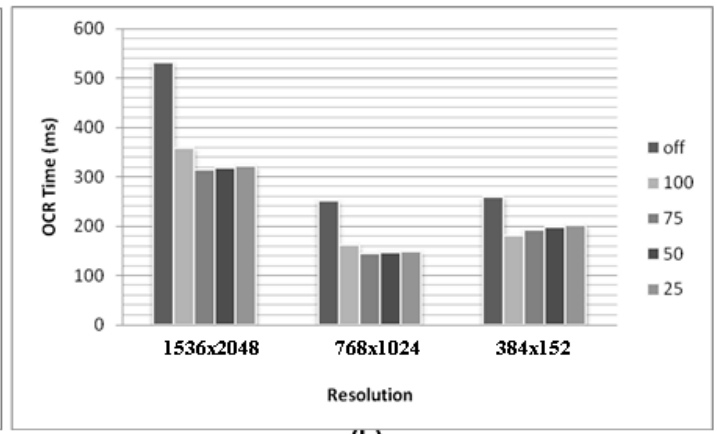

(b)

Figure 7.12 (a) OCR time for in-phone approach (b) OCR time for remote server approach 
Figure 7.13 compares OCR values for the in-phone and the remote-server approaches according to a constant resolution (768x1024) and varied compression settings. Overall, we can see significant differences in OCR time. For example, OCR time is $2347 \mathrm{~ms}$. for the in-phone approach and $252 \mathrm{~ms}$. for the remote-server approach when compression is set to "off".

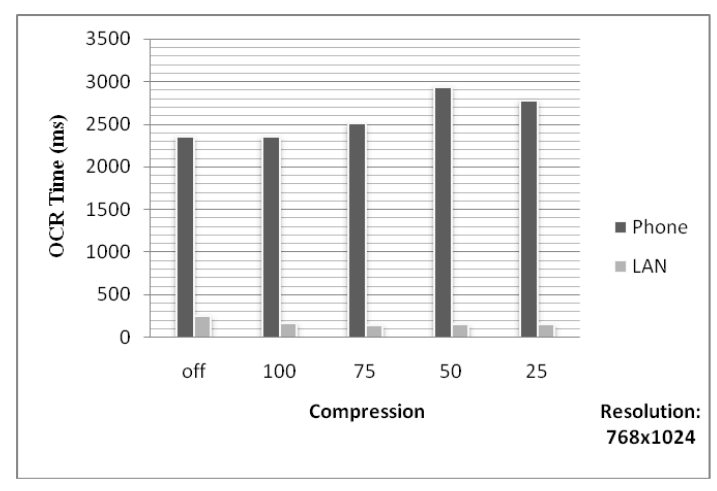

Figure 7.13 OCR time for the in-phone and the remote server approaches in $768 \times 1024$ pixel resolution

Figure 7.14 exhibits the average network delay (ND) for different resolutions and Qfactors for the remote-server approach. As expected, the overall trend shows that reducing the resolution of the image (downscaling) dramatically decreases ND. For example, downscaling an uncompressed image from 1536x2048 to $768 \times 1024$ reduces network delay from $30055 \mathrm{~ms}$ to $8874 \mathrm{~ms}$ for the remote server approach. Similar to the total execution time, Q-factor 75 considerably decreases the ND, but other $\mathrm{Q}$-factors gradually reduce the network delay.

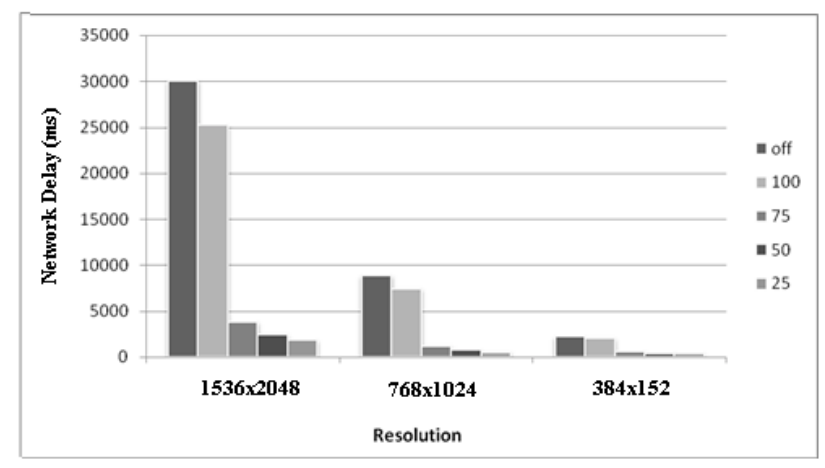

Figure 7.14 Network delay for the remote-server approach

Figure 7.15 shows that the compression and decoding delay (CDD) change for different resolutions and Q-factors. The CDD is not applicable for the tests that use uncompressed images, since compression and decoding phases are unnecessary 
for an uncompressed image. There is a similar pattern for TET, ND and CDD. As in the TET and the ND, Q-factor 75 substantially decreases the CDD, but other compression quality levels gradually decrease the TET.

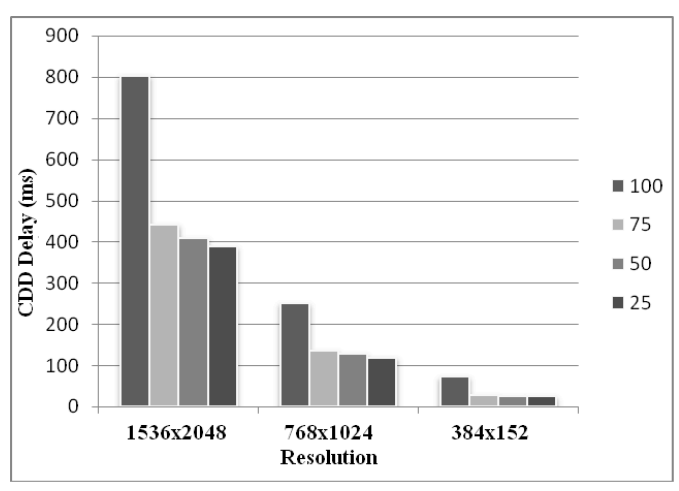

Figure 7.15 Compression and decoding delay of compressed images

\subsubsection{Comparing Accuracy Performance}

Figure 7.16(a) and Figure 7.16(b) illustrate variations in the Word Recognition Rate (WRR) and the Character Recognition Rate (CRR) for different resolutions and Qfactors, respectively. From $1536 \times 2048$ to $768 \times 1024$ resolutions, there is a slight $(2 \%)$ decrease in the WRR. The CRR does not change in 768x1024 pixel resolution. In contrast, from $768 \times 1024$ to $384 \times 512$ resolutions, the WRR and the CRR decrease considerably.

According to Figure 7.16(b), Q-factor 100 does not change WRR or CRR. Q-factor 75 slightly (1\%) decreases both WRR and CRR. Q-factor 50 and Q-factor 25 decreases WRR and CRR $2 \%$ and $3 \%$, respectively.

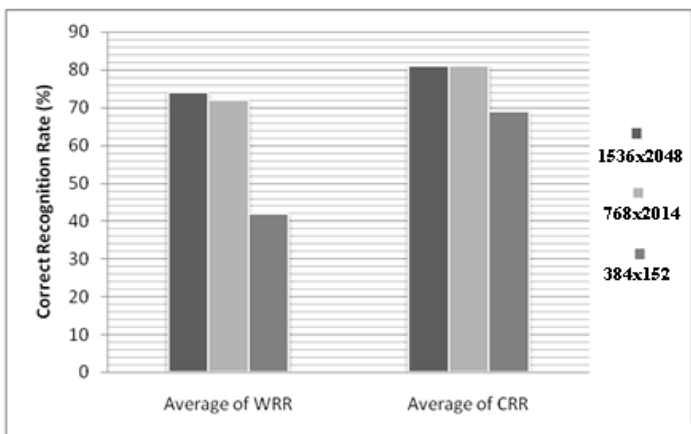

(a)

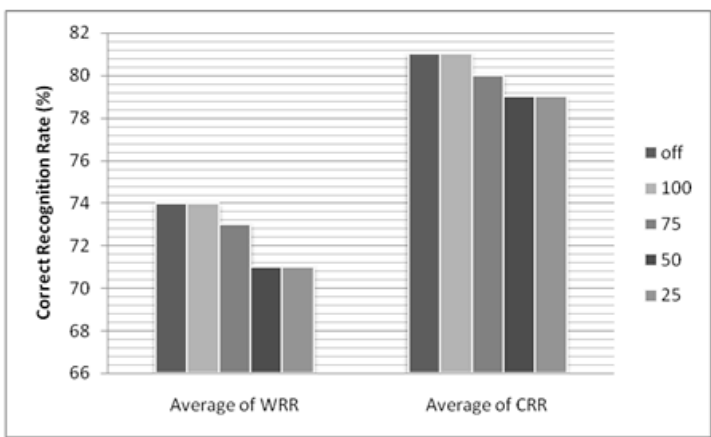

(b)

Figure 7.16 (a) WRR and CRR for varied resolutions (b) WRR and CRR for varied compressions 
In these experiments, we compare two approaches for mobile document image analysis regarding the defined speed and correct recognition performance metrics. The first approach is performing all processes completely on the mobile phone, and the second approach is performing the OCR process on the remote server.

The general observation from experiments is that for the total execution time (TET) metric, the in-phone approach outperforms the remote server approach for only uncompressed images and very high quality compressed images (Q-factor $>75)$ at high resolutions (1536x2048 and $7668 \times 1024)$. The poor TET performances of the remote-server approach at high quality images are mainly attributed to large image sizes, and lack of adequate network bandwidth. Nevertheless, the remote server approach outperforms the in-phone approach for $768 \times 1024$ and lower resolutions and 75 and lower compression quality factors.

The other major point is that the remote server approach considerably surpasses the in-phone approach in terms of OCR time, as expected. The minimum OCR time is $145 \mathrm{~ms}$ for the server approach and $2248 \mathrm{~ms}$ for the in-phone approach.

We also verify that, compression and downscaling (reducing the resolution) decrease the network delay (ND) for server approaches, since these operations reduce transferred image size. The network delay is the biggest component of TET for the server approach, while the OCR time is the biggest component of TET for the in-phone approach.

There is also a compression and decoding delay (CDD) that is applicable for only compressed images. Because of the CDD delay, compression is not necessary for the in-phone approach. On the other hand, it is very favorable for the server approach since compression decreases the transferred image size. According to the results of the remote server experiments, Q-factor 75 considerably decreases CDD, ND, OCR and TET, while it slightly decreases WRR and CRR. Thus, Q-factor 75 is preferable for the remote server approaches.

Reducing the image resolution significantly decreases the Total Execution Time (TET) for the in-phone and the remote server approaches. 2-factor downscaled images at $768 \times 1024$ pixels yield the minimum TET for the in-phone OCR. 4-factor downscaled images at $384 \times 512$ pixels yield the minimum TET for the server OCR, but there are small differences between TET values of $384 \times 512$ pixel resolution images and $768 \times 1024$ pixel resolution images. On the other hand, the average word recognition rate (WRR) of $768 \times 1024$ pixel resolution images is $30 \%$ higher than $384 \times 512$ pixel resolution images. Therefore, $768 \times 1024$ pixel resolution images are preferable for all the three approaches with respect to speed and acceptable correct recognition rates.

In conclusion, in our experiments uncompressed $768 \times 1024$ pixel resolution images appear to be the most preferable for the in-phone OCR approach regarding speed and recognition metrics. The total execution time is $2531 \mathrm{~ms}$ and OCR time $2347 \mathrm{~ms}$ 
for this setting. For the remote server approach, $768 \times 1024$ pixel resolution images compressed with $\mathrm{Q}$-factor 75 is the fastest setting with only $1 \%$ loss at correct recognition rates. For this setting, the total execution times are $1637 \mathrm{~ms}$, OCR time is $145 \mathrm{~ms}$ These results show that the remote server approach performs better than the in-phone approach for the most favorable settings of each approach. Even when the server approach contains the extra compression, decoding and network delays, it outperforms the in-phone approach since the reduced OCR time compensates for these extra delays.

\subsection{Multi-criteria Product Recommendation Experiments}

We test the multi-criteria recommender component with four different recommendation algorithms to understand differences among their results. The tested algorithms are the single criterion ranking, weighted sum, PROMETHEE II [150], and ELECTRE III [161] algorithms.

A case study is developed to compare each method. Six different toothpaste $\left(A_{1}, A_{2}\right.$, $\left.\ldots, A_{6}\right)$ are selected as product alternatives. Five criteria $\left(C_{1}, C_{2}, \ldots, C_{5}\right)$ are considered to compare these alternatives. The criteria weights are assigned using the AHP method suggested by Saaty [146].

Table 7.6 shows these criteria, the weight of importance $\left(w_{j}, j=1,2, \ldots, 5 ; \Sigma_{j} w_{j}=1\right)$ assigned to each of the criteria, as well as their preference directions. $C_{1}$ criterion (price) has the "minimize" preference direction criterion, since the decision maker wants to minimize the value of this criterion. Other criteria have the "maximize" preference direction, which should be maximized for the benefit of the decision maker.

Table 7.6 Evaluative criteria, criteria weights, and preference directions

\begin{tabular}{llll}
\hline Symbol & Criteria Name & Weight $\left(\mathbf{w}_{\mathbf{j}}\right)$ & $\begin{array}{l}\text { Preference } \\
\text { Direction }\end{array}$ \\
\hline$C_{1}$ & Price & 0.25 & Minimize \\
\hline$C_{2}$ & Taste & 0.12 & Maximize \\
\hline$C_{3}$ & Sensitivity & 0.40 & Maximize \\
\hline$C_{4}$ & Naturalness & 0.08 & Maximize \\
\hline$C_{5}$ & Whitening & 0.15 & Maximize \\
\hline
\end{tabular}


Table 7.7 shows the decision matrix, which includes the quantitative evaluation of each product alternative according to each criterion. $a_{i j}$ define the quantitative evaluation, which indicates the performance of the alternative $A_{i}$ with respect to criterion $\mathrm{C}_{\mathrm{j}}$.

Table 7.7 The decision matrix

\begin{tabular}{cccccc}
\hline & C1 & C2 & C3 & C4 & C5 \\
\hline A1 & 8 & 9 & 10 & 10 & 7 \\
\hline A2 & 10 & 10 & 8 & 8 & 10 \\
\hline A3 & 3 & 6 & 6 & 7 & 6 \\
\hline A4 & 6 & 8 & 7 & 8 & 9 \\
\hline A5 & 8 & 9 & 10 & 8 & 9 \\
\hline A6 & 7 & 7 & 7 & 6 & 7 \\
\hline
\end{tabular}

According to the decision matrix, $\mathrm{A} 3$ alternative requires the minimum cost of price (criterion $\mathrm{C} 1$ ), but corresponds to the minimum benefit of taste (criterion $\mathrm{C} 2$ ), sensitivity (criterion C3), and whitening (criterion C5). However, A2 alternative has the maximum cost of price (criterion $\mathrm{C} 1$ ), but corresponds to the maximum benefit of taste (criterion C2) and whitening (criterion C5). These examples demonstrate that the optimal solution is not obvious for this use case.

Accordingly, we apply four different decision algorithms on this use case. The first algorithm is the single criterion ranking algorithm, which is the simplest recommendation algorithm that ranks products with respect to a single criterion. The second algorithm is the weighted sum algorithm that uses criteria weights in addition to the values of multiple criteria. The third and fourth algorithms are PROMETHEE II [150], and ELECTRE III [161] algorithms, which are sophisticated algorithms that uses additional parameters, named criteria thresholds.

The criteria thresholds enable to reflect the decision maker's demands more precisely, which are the indifference threshold $q_{j}$, the preference threshold $p_{j}$ and the veto threshold $v_{j}$ as mentioned in Chapter 5.3.1. The criteria thresholds for this use case are given in Table 7.8.

- If the difference between performances of two alternatives is lower than the indifference threshold, $\mathrm{q}_{\mathrm{j}}$, the user does not have preference between two product alternatives for a given criterion.

- If the difference between performances of two alternatives is above than the preference threshold, $\mathrm{p}_{\mathrm{j}}$, the user prefers one alternative to another for a given criterion. 
- If the difference between performances of two alternatives is above than the veto threshold, $v_{\mathrm{j}}$, the user "definitely" prefers one alternative to another for a given evaluative criterion.

Table 7.8 The criteria thresholds

\begin{tabular}{lccccc}
\hline & C1 & C2 & C3 & C4 & C5 \\
\hline Preference & 5 & 4 & 2 & 2 & 5 \\
\hline Indifference & 2 & 1 & 0 & 1 & 1 \\
\hline Veto & 10 & 4 & 3 & 1 & 2 \\
\hline
\end{tabular}

\subsubsection{Results of the Single Criterion Ranking Algorithm}

As mentioned above, the single criterion ranking algorithm ranks products with respect to a single criterion. In this use case, this algorithm ranks toothpastes considering only the "price" criterion, from the cheapest one to the most expensive one. As seen in Table 7.9, the optimal choice is the product alternative $A_{3}$.

Table 7.9 The results of the single criterion ranking algorithm

\begin{tabular}{ccc}
\hline & C $_{1}$ Score & Rank \\
\hline A1 & 9 & 5 \\
\hline A2 & 10 & 6 \\
\hline A3 & 3 & 1 \\
\hline A4 & 6 & 2 \\
\hline A5 & 8 & 4 \\
\hline A6 & 7 & 3 \\
\hline
\end{tabular}

\subsubsection{Results of the Weighted Sum Algorithm}

The weighted sum algorithm ranks products by using the multiple criteria. This algorithm also uses the criteria weights of the user. In the weighted sum algorithm, each criteria value is multiplied by that criteria's weight, and those products are summed to calculate the score. Table 7.10 shows the results of the weighted sum algorithm. 
Table 7.10 The results of the weighted sum algorithm

\begin{tabular}{lccccccc}
\hline & C1 & C2 & C3 & C4 & C5 & Score & Rank \\
\hline A1 & 8 & 9 & 10 & 10 & 7 & 4.93 & 2 \\
\hline A2 & 10 & 10 & 8 & 8 & 10 & 4.04 & 4 \\
\hline A3 & 3 & 6 & 6 & 7 & 6 & 3.83 & 5 \\
\hline A4 & 6 & 8 & 7 & 8 & 9 & 4.25 & 3 \\
\hline A5 & 8 & 9 & 10 & 8 & 9 & 5.07 & 1 \\
\hline A6 & 7 & 7 & 7 & 6 & 7 & 3.42 & 6 \\
\hline Weight & 0.25 & 0.12 & 0.4 & 0.08 & 0.15 & & \\
\hline
\end{tabular}

\subsubsection{Results of the ELECTRE III Algorithm}

The ELECTRE III [161] algorithm uses outranking relations between the product alternatives, which are taken two at a time. According to this algorithm, an alternative $A_{i}$ outranks the alternative $A_{j}$, if $A_{i}$ shows better or at least equal performance $A_{j}$ regarding the majority of criteria. The ELECTRE III algorithm uses criteria weights and criteria thresholds to calculate these outranking relations. Table 7.11 shows that the result of the ELECTRE III algorithm for the use case.

Table 7.11 The results of the ELECTRE III algorithm

\begin{tabular}{lcccccc}
\hline & C1 & C2 & C3 & C4 & C5 & Rank \\
\hline A1 & 8 & 9 & 10 & 10 & 7 & 1 \\
\hline A2 & 10 & 10 & 8 & 8 & 10 & 4 \\
\hline A3 & 3 & 6 & 6 & 7 & 6 & 6 \\
\hline A4 & 6 & 8 & 7 & 8 & 9 & 3 \\
\hline A5 & 8 & 9 & 10 & 8 & 9 & 2 \\
\hline A6 & 7 & 7 & 7 & 6 & 7 & 5 \\
\hline Weight & 0.25 & 0.12 & 0.4 & 0.08 & 0.15 & \\
\hline Preference & 5 & 4 & 2 & 2 & 5 & \\
\hline Indifference & 2 & 1 & 0 & 1 & 1 & \\
\hline Veto & 10 & 4 & 3 & 3 & 8 & \\
\hline
\end{tabular}




\subsubsection{Results of the PROMETHEE II Algorithm}

Similar to the ELECTRE III method, the PROMETHEE II [150] algorithm uses criteria weights and criteria thresholds. This algorithm is based on the binary comparison of alternatives considering the deviations of alternatives regarding each criterion.

The results of the PROMETHEE II algorithm are given in Table 7.12.

Table 7.12 Results of the PROMETHEE II algorithm

\begin{tabular}{lcccccc}
\hline & C1 & C2 & C3 & C4 & C5 & Rank \\
\hline A1 & 8 & 9 & 10 & 10 & 7 & 1 \\
\hline A2 & 10 & 10 & 8 & 8 & 10 & 3 \\
\hline A3 & 3 & 6 & 6 & 7 & 6 & 6 \\
\hline A4 & 6 & 8 & 7 & 8 & 9 & 4 \\
\hline A5 & 8 & 9 & 10 & 8 & 9 & 2 \\
\hline A6 & 7 & 7 & 7 & 6 & 7 & 5 \\
\hline Weight & 0.25 & 0.12 & 0.4 & 0.08 & 0.15 & \\
\hline Preference & 5 & 4 & 2 & 2 & 5 & \\
\hline Indifference & 2 & 1 & 0 & 1 & 1 & \\
\hline Veto & 10 & 4 & 3 & 3 & 8 & \\
\hline
\end{tabular}

\subsubsection{Discussion of the Results of the Decision Making Algorithms}

In this section, the results of the four decision making algorithm (the single criterion ranking, the weighted sum algorithm, the ELECTRE III algorithm, and the PROMETHEE II algorithm) are calculated regarding the given use case data.

In this use case, different toothpaste alternatives are selected as product alternatives and five criteria are considered to compare these alternatives. The criteria weights, performances of alternatives with respect to each criterion, and criteria thresholds (indifference, preference, and veto) are given in the use case. However, some algorithms do not require all of these data. Table 7.13 shows the comparison of the used data by each algorithm. 
Table 7.13 Used data by each algorithm

\begin{tabular}{lcccc}
\hline & $\begin{array}{c}\text { Single } \\
\text { Criterion }\end{array}$ & $\begin{array}{c}\text { Multi- } \\
\text { Criteria }\end{array}$ & $\begin{array}{c}\text { Criteria } \\
\text { Weights }\end{array}$ & $\begin{array}{c}\text { Criteria } \\
\text { Threshold } \\
\text { s }\end{array}$ \\
\hline $\begin{array}{l}\text { Single Criterion } \\
\text { Ranking }\end{array}$ & $\mathrm{X}$ & & & \\
\hline Weighted Sum & $\mathrm{X}$ & $\mathrm{X}$ & \\
\hline ELECTRE III & $\mathrm{X}$ & $\mathrm{X}$ & $\mathrm{X}$ \\
\hline PROMETHEE II & $\mathrm{X}$ & $\mathrm{X}$ & $\mathrm{X}$ \\
\hline
\end{tabular}

According to the results, each algorithm gives a different ranked list of the product alternatives as shown in Table 7.14.

As seen in Table 7.14, for the single criterion ranking algorithm, $A 3$ is the best product for the decision maker according to the price $\left(\mathrm{C}_{1}\right)$ criterion, although it is the worst product regarding taste (C2), sensitivity (C3), and whitening (C5) criteria.

Table 7.14 shows that the best product is $A_{5}$ for the weighted sum algorithm, while $A_{5}$ is ranked in fourth in the single criterion ranking algorithm. In addition, the best product for the single criterion ranking algorithm, $A_{3}$, is in the fifth rank for the weighted sum method. For the weighted sum algorithm, utilizing the multiple criteria enables compensation of the low-value criteria by the high-value criteria.

Table 7.14 Comparison of the results of the decision algorithms

\begin{tabular}{ccccc}
\hline & $\begin{array}{c}\text { Single } \\
\text { Criterion } \\
\text { Rank }\end{array}$ & $\begin{array}{c}\text { Weighted } \\
\text { Sum Rank }\end{array}$ & $\begin{array}{c}\text { ELECTRE III } \\
\text { Rank }\end{array}$ & $\begin{array}{c}\text { PROMETHE II } \\
\text { Rank }\end{array}$ \\
\hline A1 & 5 & 2 & 1 & 1 \\
\hline A2 & 6 & 4 & 4 & 3 \\
\hline A3 & 1 & 5 & 6 & 6 \\
\hline A4 & 2 & 3 & 3 & 4 \\
\hline A5 & 4 & 1 & 2 & 2 \\
\hline A6 & 3 & 6 & 5 & 5 \\
\hline
\end{tabular}


The comparison of the results of the single criterion ranking algorithm and the weighted sum algorithm implies that the weighted sum algorithm presents more optimal choices than the single criterion ranking algorithm, since it reflects the preference of the consumer about other criteria, such as taste, naturalness, sensitivity and whitening, and also the criteria weights calculated through the consumer's preferences.

As seen in Table 7.14, $A_{1}$ is the optimal choice for both ELECTRE III and PROMETHEE II algorithms, while $A_{5}$ is the optimal choice according to the weighted sum algorithm. Although all of these three algorithms are multi-criteria decision making algorithms that use multiple criteria and users' weights for these criteria, ranking results of them are different. Since the ELECTRE III and PROMETHEE II algorithms also use the preference, indifference and veto thresholds, they better reflect the consumer's preferences. For example, the veto threshold of the naturalness criterion $\left(\mathrm{C}_{4}\right)$ is given as 1 , and the ELECTRE III and PROMETHEE II algorithms use this information to calculate the ranked alternatives list. Values of the $\mathrm{C}_{4}$ for product $\mathrm{A}_{1}$ and $\mathrm{A}_{5}$ are 10 and 8 , respectively. According to definition of the veto threshold, the difference between performances of two $A_{1}$ and $A_{5}$ is above than the veto threshold of the criterion $\mathrm{C}_{4}, 1$, accordingly the user "definitely" prefers $A_{1}$ to $A_{5}$ for the criterion $C_{4}$. Then, $A_{1}$ outranks $A_{5}$ in the ELECTRE III and PROMETHEE II algorithms, but $A_{5}$ outranks $A_{1}$ in the weighted sum algorithm, since it does not utilize the criteria thresholds, specifically the veto threshold for this use case.

Overall, the results of the multi-criteria product recommendation experiments show that multi-criteria algorithms give more accurate results regarding the consumers' preferences. In addition, utilization of the criteria thresholds in multi-criteria decision making algorithms increase the decision quality, since they reflect users' preferences more precisely. 


\section{CHAPTER 8}

\section{RESEARCH EVALUATION}

This chapter summarizes the research activities and results, and argues them against Design Science Research (DSR) guidelines of Hevner, et al. [25] in order to validate this research as a DSR project as described by [25].

The rest of this chapter is formed around the seven guidelines of DSR. In order to address each guideline, we described that how a guideline is translated and implemented in our research.

\subsection{Hevner's DSR Guidelines}

\subsubsection{Design as an Artifact}

The first guideline, design as an artifact, defines a compulsory requirement of DSR research in relation to the outcome of the entire research [25]. The authors described this requirement as "The result of design-science research in IS is, by definition, a purposeful IT artifact created to address an important organizational problem. It must be described effectively, enabling its implementation and application in an appropriate domain." (Hevner, et al. [25], p82)

The "purposeful IT artifact" of this research is the MobileCDP framework, which is presented in Chapter 4. This artifact is constructed on the consumer decision process (CDP) and empowered with the participatory sensing approach to address challenges faced by the consumers in their activities, and facilitates the development of the mobile information systems for the entire CDP that includes the problem recognition, information search, evaluation of alternatives, purchase decision, and post-purchase evaluation stages. MobileCDP provides twelve components to support CDP, ten of them are facilitating components that target individual stages of CDP to help consumers in these stages, and two of them are unifying components that are responsible for integration and coordination among the facilitating components.

The other artifact is the prototype introduced in Chapter 6 . This prototype mobile application is an instantiation of MobileCDP, and shows applicability and feasibility 
of the framework. The prototype consists of twelve modules, and each module of the prototype corresponds to the relevant component of the MobileCDP framework. However, a developed prototype module is a limited instantiation of the corresponding component of MobileCDP. Therefore, the developed prototype is to be understood a proof of concept and not a fully functional implementation.

\subsubsection{Problem Relevance}

The second guideline, problem relevance, addresses the need that DSR research must be relevant to a business problem. [25] defines this guideline as "The objective of design-science research is to develop technology-based solutions to important and relevant business problems." (Hevner, et al. [25], p83)

The relevant business problem for our research is that consumers experience various difficulties as part of their activities due to cognitive limitations such as limited computational capacities and limited memory [2], and these limitations decrease the decision quality and increase time spent, costs, and cognitive effort related to shopping $[2-5,7]$.

\subsubsection{Design Evaluation}

Design evaluation guideline describes the need for the evaluation of the designed artifact. Hevner, et al. [25] states that "The utility, quality, and efficacy of a design artifact must be rigorously demonstrated via well-executed evaluation methods." (Hevner, et al. [25], p83). Accordingly, we implement a prototype mobile application based on the designed artifact, the MobileCDP framework. Chapter 6 presents the prototype. Then, a usability evaluation is conducted to evaluate the effect of the developed prototype on user performance and user experience, as well as to assess whether it is effective, efficient and satisfactory.

As reported in Chapter 7, the results of the evaluation show that the prototype is functional, useful, well integrated, and easy to use, which implies that the framework can be successfully utilized as an integrated mobile information system. In addition, the results show that the prototype decreases the time spent and cost in the decision process, reduces the cognitive effort of users.

\subsubsection{Research Contributions}

As the fourth guideline, research contribution emphasizes that clear contribution is needed for a DSR project [25]. Hevner, et al. [25] also state that such a contribution can be a design artifact, design foundation, and/or design methodology.

As outlined in Chapter 3.3.1, the primary contribution of this research is the artifact, the MobileCDP framework, which is the first study based on an integrated and holistic approach addressing all stages of the consumer decision process according to the best of our knowledge, and consistent with recent research [19]. Literature review presented in Chapter 2 shows that existing studies focus on specific tasks in 
the consumer decision process, such as price comparison, review search, and creating shopping lists. This artifact provides the required components of a mobile information system to be designed to support consumers in the decision process.

In addition, this research contributes to the body of knowledge in the area of mobile information systems for consumers by providing an integrated and holistic approach. This approach includes the facilitating components that assist a stage of the consumer decision process, and the unifying components that integrate and coordinate the facilitating components. The evaluation of the developed prototype shows that such an approach provides benefits to consumers by enabling utilization of more information compared to stand-alone solutions developed for individual stages. Regarding [25], the prototype implementation is an instantiation of the artifact, and it is another secondary contribution of this research. The proposed Text Clustering Based Multi-frame Integration (TB-MFI) and Knowledge Based Correction (KBC) algorithms are other contributions of this research.

\subsubsection{Research Rigor}

This guideline emphasizes the importance of research rigor in DSR. [25] state that "Design- science research relies upon the application of rigorous methods in both the construction and evaluation of the design artefact." (Hevner, et al. [25], p87) Then, Hevner, et al. [25] specify this requirement by arguing, "... rigor is derived from the effective use of the knowledge base - theoretical foundations and research methodologies." (Hevner, et al. [25], p88)

Chapter 3 describes the research methodology of this research. Overall, we chose Design Science Research [25] as the research methodology, and Design Science Research Methodology (DSRM) Process [26] as the underlying research process as shown in Figure 8.1.

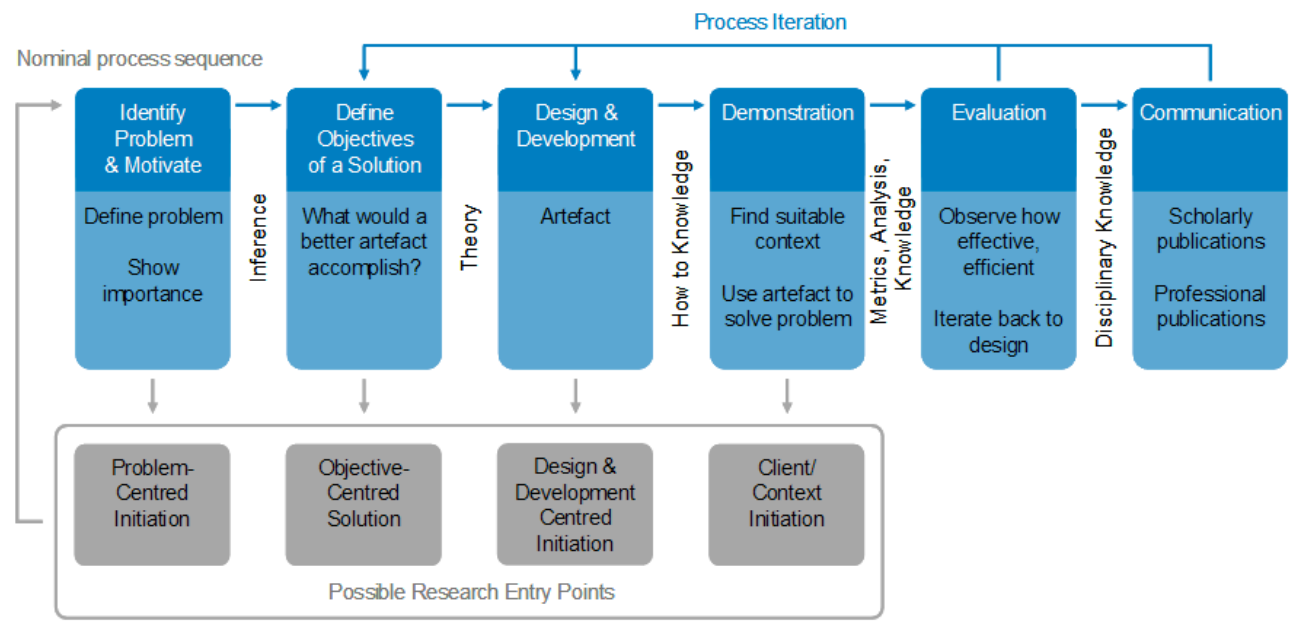

Figure 8.1 The DSRM process (adapted from [26]) 
As mentioned in Chapter 3.2.1, Peffers, et al. [26] identify four possible research entry points: problem-centered initiation, objective centered solution, design and development centered initiation, and client/context initiation. Considering these entry points, the entry point of this research is the problem-centered initiation. We identified a problem in existing literature, and then investigated this problem in the research process.

Figure 8.2 summarizes how the DSRM process of Peffers, et al. [26] applies to the steps undertaken as part of our design science research.

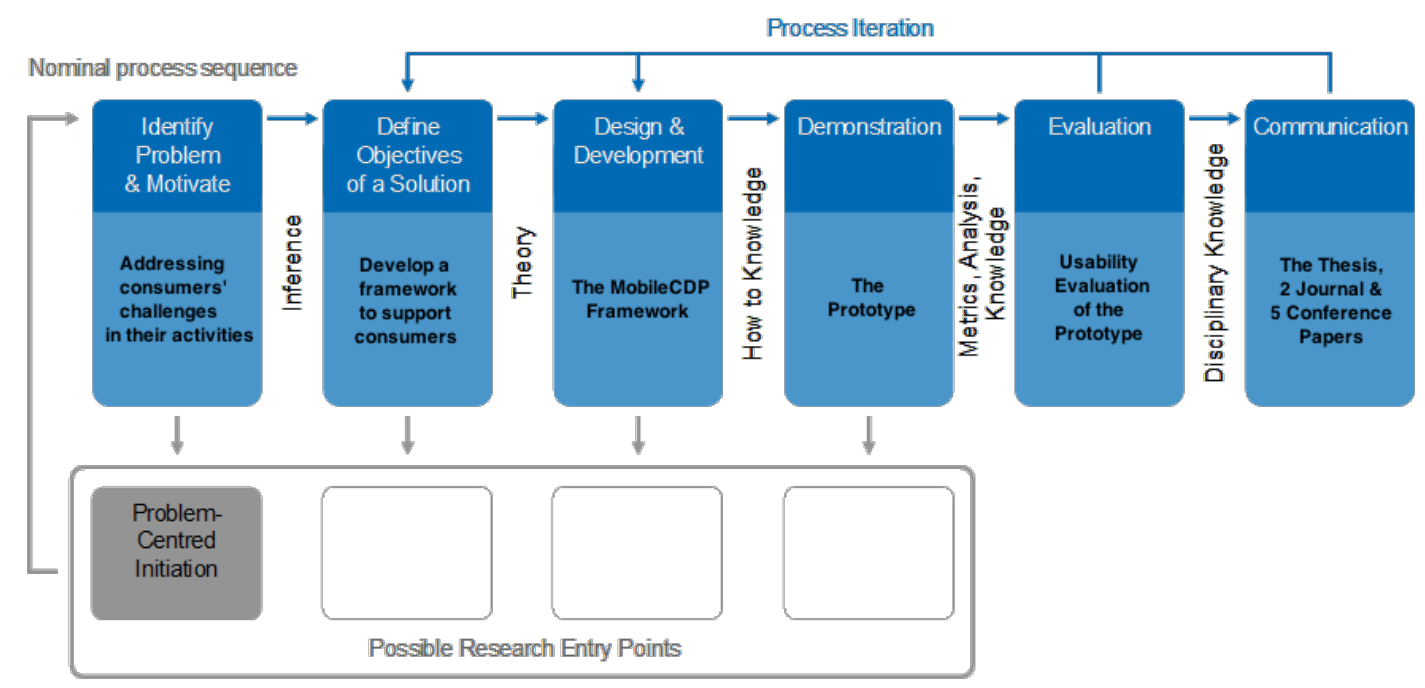

Figure 8.2 The DSRM Process of this research

In addition, Table 8.1 shows corresponding thesis chapters for each activity of the DSRM process.

\subsubsection{Design as a Search Process}

As stated by [25, 26], DSR is an iterative search process to discover an effective solution to a problem.

The MobileCDP framework is designed by a thorough analysis of literature and identifying components of the framework. Examining literature, identifying new components, and improving existing components constitute an iteration for this research. For example, in early phases of the research, the MobileCDP framework consisted of five components, and each component corresponded to a stage of the consumer decision process. The data collector module was added in the next iteration, and the search process has progressed in this way. The continuous examination of the consumer behavior and mobile information systems literature led to a continuous improvement of the MobileCDP framework. 
Table 8.1 Mapping thesis chapters to the DSRM process of Peffers, et al. [26]

\begin{tabular}{|c|c|c|}
\hline Activity \# & Activity & Thesis \\
\hline \multirow{2}{*}{ Activity 1} & \multirow{2}{*}{ Identify Problem \& Motivate } & Chapter 1 - Introduction \\
\hline & & Chapter 2 - Literature Review \\
\hline \multirow{2}{*}{ Activity 2} & \multirow{2}{*}{ Define Objectives of Solution } & Chapter 4 - The MobileCDP Framework \\
\hline & & Chapter 5 - Participatory Sensing \\
\hline \multirow{2}{*}{ Activity 3} & \multirow{2}{*}{ Design and Development } & Chapter 4 - The MobileCDP Framework \\
\hline & & Chapter 5 - Participatory Sensing \\
\hline Activity 4 & Demonstration & Chapter 6 - Prototype Implementation \\
\hline Activity 5 & Evaluation & Chapter 7 - Prototype Evaluation \\
\hline Activity 6 & Communication & Chapter 8.2.7 - List of Publications \\
\hline
\end{tabular}

\subsubsection{Communication of Research}

Hevner, et al. [25] emphasize that "Design-science research must be presented effectively both to technology-oriented as well as management-oriented audiences."

Our research includes both technology-oriented (e.g., mobile information systems) and management-oriented (e.g., consumer decision process) concepts. Although the primary audience of this research is technology-oriented academics, it is also relevant to management-oriented audiences.

The primary communication of this research is this $\mathrm{PhD}$ thesis, which is a detailed and comprehensive piece of communication of this research. As explained in Chapter 3.3, this thesis is compatible with the DSR publication schema, which is suggested by Gregor and Hevner [27] to appropriately communicate design science research projects

2 submitted journal papers and 5 published conference papers are secondary communication of this research, which are listed in below.

- Özarslan, S., Eren P.E. (2015). MobileCDP: A mobile framework for the consumer decision process. Manuscript submitted for review.

- Özarslan, S., Eren P.E. (2015). A Text Clustering Based Multi-Frame Integration Method for Low Quality Mobile Phone Captured Video OCR. Manuscript submitted for review.

- Özarslan, S., Eren P.E., "Multi-frame Knowledge Based Text Enhancement for Mobile Phone Captured Videos," Proc. SPIE 9030, Mobile Devices and 
Multimedia: Enabling Technologies, Algorithms, and Applications 2014, (2014).

- Özarslan, S., Eren P.E., "Text recognition and correction for automated data collection by mobile devices," Proc. SPIE 9027, Imaging and Multimedia Analytics in a Web and Mobile World 2014, (2014).

- Özarslan, S., Eren P.E., "Comparison of Approaches for Mobile Document Image Analysis Using server supported smartphones," Proc. SPIE 9023, Digital Photography X 2014, (2014).

- Özarslan, S., Eren, P. E., "A Mobile Participatory Framework for the Consumer Decision Process," Proc. Seventh International Conference on Next Generation Mobile Apps, Services and Technologies (NGMAST), 93-98 (2013).

- Özarslan, S., Eren, P. E. "Katılımcı Algılama Uygulamalarında Otomatik Veri Toplama İçin Karakter Tanıma ve Düzeltme", Signal Processing and Communications Applications Conference (SIU), (2013).

\subsection{Chapter Summary}

In this chapter, different elements of our research are revisited, and these elements evaluated against the Seven Design Science Research Guideline suggested by Hevner, et al. [25] in order to satisfy the requirement of a research evaluation, which is part of the DSR process.

Overall, this research satisfies the requirements of all seven guidelines of Hevner, et al. [25], and meets the criteria for a valid design science research project. In addition, this research addresses all activities of the Design Science Research Methodology Process of Peffers, et al. [26]. Furthermore, regarding Gregor and Hevner [27], this thesis document all aspects of the research process as well as the resulting artifact, the MobileCDP framework. 


\section{CHAPTER 9}

\section{CONCLUSION AND FUTURE RESEARCH}

\subsection{Conclusion}

Consumer behavior research addresses challenges in consumer activities, and the consumer decision process is an essential part of the consumer behavior. While following the process, consumers experience difficulties due to cognitive limitations, and these difficulties lead to loss of time and money. Several research studies on mobile information systems are proposed to overcome the difficulties experienced in the decision process, but they are primarily focused on the individual stages.

In this thesis research, we design a mobile framework, MobileCDP. The motivation behind this research is a literature review that identified a gap where the existing solutions do not support consumers in all stages of the consumer decision process. Unlike the previous studies, the main goal of our research is to provide a mobile framework by designing, integrating and evaluating components for mobile information systems to be developed to support consumers in all stages of the consumer decision process. This research also reveals that such a solution has intensive data requirements, and demonstrates the importance of participatory sensing in the context of supporting consumers on their activities.

This research makes several contributions to the current literature. First, to the best of the authors' knowledge, and consistent with recent research [19], MobileCDP is the first study based on an integrated and holistic approach addressing all stages of the consumer decision process, while existing studies in the literature focus on specific stages. As the main artifact of this research, the primary contribution is the proposed MobileCDP framework.

The present study provides the required components of a mobile information system to be designed to support consumers in the decision process. Not only the facilitating components, but also the unifying ones are incorporated into MobileCDP in support of the holistic approach, also enabling utilization of more information compared to stand-alone solutions developed for individual stages. The integrated and holistic approach provided by these components contributes to the body of knowledge in the area of mobile information systems for consumers. 
Second, the proposed algorithms that provide automated data collection for the framework are also artifacts of this research, and contributes to the body of knowledge of the participatory sensing and document recognition domains. A Text Based Multi-Frame Integration Algorithm (TB-MFI) is developed to integrate information in the video frames in order to improve the data recognition accuracy, and a Knowledge Based Correction (KBC) algorithm is developed in support of the Optical Character Recognition (OCR) to correct inaccurately recognized information. Taken together, these algorithms are used to extract information from store receipt images and videos captured by mobile devices of consumers, and eliminate errors in the extracted information. The results of the experiments show that these algorithms increase the accurate character recognition rate from $73 \%$ to $98 \%$.

Third, a prototype of the MobileCDP framework is developed to show the applicability and benefits of the framework. Experiments are also conducted to evaluate the prototype. According to the results of the usability test, users found the features of the prototype useful, and the system easy to use. Users agreed to use the prototype frequently, and they stated that the functions of the prototype are well integrated. These results show that the integrated and holistic approach of MobileCDP is applicable. Moreover, statistical analysis of the task completion times and selection errors prove that the prototype is time and cost saving, and reduces the cognitive effort of the consumer. Taken together, these results demonstrate that MobileCDP provides support for the consumer decision process to the benefit of consumers, and also highlight that such mobile information systems address the challenges experienced by consumers.

\subsection{Future Research}

This research may serve as an extensible foundation for future studies on mobile information systems assisting consumers in their decision processes. As an ongoing research area, mobile technologies in support of consumers require more research efforts to gain broader adaptation of solutions on the mobile domain for addressing challenges of consumers, which are explored in the consumer behavior domain.

The main limitation of this research is that the developed modules of the prototype have restricted functionality compared to the corresponding proposed components in MobileCDP. Even though the prototype does not contain all the functionalities described in the framework, the participants of the experiments stated that they would like to use the prototype frequently. Further research may extend the framework to provide a greater degree of assistance for the consumer decision process. In addition, specific algorithms designed for individual stages and integrated as modules within the framework may help further automate the process. Furthermore, future research should focus on privacy and security aspects of mobile solutions that proposed to address consumers' challenges. 


\section{REFERENCES}

[1] D. Hawkins, D. Mothersbaugh, and R. Best, Consumer Behavior: Building Marketing Strategy. Boston, MA: McGraw-Hill//rwin, 2012.

[2] J. R. Bettman, E. J. Johnson, and J. W. Payne, "Consumer decision making," in Handbook of consumer behavior, T. S. Robertson and H. H. Kassarjian, Eds., ed Englewood Cliffs, N.J.: Prentice-Hall, 1991, pp. 50-84.

[3] J. D. Lindquist and M. J. Sirgy, Shopper, Buyer, and Consumer Behavior: Theory, Marketing Applications and Public Policy Implications. Florence, KY: Cengage Learning, 2009.

[4] J. Engel, R. Blackwell, and P. Miniard, Consumer Behavior. New York: The Dryden Press, 1995.

[5] L. P. Jarvis and J. B. Wilcox, "Evoked set size: some theoretical foundations and empirical evidence," in Combined Proceedings of the Fall Conference of the American Marketing Association, 1973, pp. 236-40.

[6] R. Kumra, Consumer Behaviour. Mumbai: Himalaya Publishing House, 2010.

[7] J. W. Alba and J. W. Hutchinson, "Memory and decision making," Handbook of consumer behavior, pp. 1-49, 1991.

[8] U. Varshney, "Mobile health: Four emerging themes of research," Decision Support Systems, vol. 66, pp. 20-35, 10// 2014.

[9] R. Tesoriero, J. A. Gallud, M. Lozano, and V. M. R. Penichet, "Enhancing visitors' experience in art museums using mobile technologies," Information Systems Frontiers, vol. 16, pp. 303-327, 2014.

[10] S. Karpischek, F. Michahelles, and E. Fleisch, "my2cents: enabling research on consumer-product interaction," Personal Ubiquitous Comput., vol. 16, pp. 613-622, 2012. 
[11] L. Deng and L. Cox, "LiveCompare: grocery bargain hunting through participatory sensing," in HotMobile '09: Proceedings of the 10th workshop on Mobile Computing Systems and Applications, Santa Cruz, California, 2009, pp. 1-6.

[12] S. Sehgal, S. S. Kanhere, and C. T. Chou, "MobiShop: Using Mobile Phones for Sharing Consumer Pricing Information," in Intl. Conference on Distributed Computing in Sensor Systems, Santorini, Greece, 2008.

[13] F. M. Nicosia, "Consumer Decision Process: Marketing and Advertising Implications, Englewood Cliffs., 13-28," ed: NJ: Prentice Hall, 1966.

[14] J. A. Howard and J. N. Sheth, The theory of buyer behavior: New York: John Wiley \& Sons, 1969.

[15] J. F. Engel, D. T. Kollat, and R. D. Blackwell, Consumer Behavior: New York: Holt, Rinehart \& Winston, 1968.

[16] T. S. Teo and Y. D. Yeong, "Assessing the consumer decision process in the digital marketplace," Omega, vol. 31, pp. 349-363, 2003.

[17] J. Clarke, "Experiences as gifts: from process to model," European Journal of Marketing, vol. 42, pp. 365-389, 2008.

[18] P. Kotler, Marketing Management: The Millennium Edition. Upper Saddle River, NJ: Pearson Custom Pub., 2000.

[19] W. Z. Khan, Y. Xiang, M. Y. Aalsalem, and Q. Arshad, "Mobile phone sensing systems: a survey," Communications Surveys \& Tutorials, IEEE, vol. 15, pp. 402-427, 2013.

[20] J. Burke, D. Estrin, M. Hansen, A. Parker, N. Ramanathan, S. Reddy, et al., "Participatory sensing," in Workshop on World-Sensor-Web (WSW'06): Mobile Device Centric Sensor Networks and Applications, Boulder, CO, USA, 2006, pp. 117-134.

[21] S. S. Kanhere, "Participatory sensing: Crowdsourcing data from mobile smartphones in urban spaces," in Distributed Computing and Internet Technology, ed: Springer, 2013, pp. 19-26. 
[22] J. Reilly, S. Dashti, M. Ervasti, J. D. Bray, S. D. Glaser, and A. M. Bayen, "Mobile Phones as Seismologic Sensors: Automating Data Extraction for the iShake System," 2013.

[23] E. D'Hondt, M. Stevens, and A. Jacobs, "Participatory noise mapping works! An evaluation of participatory sensing as an alternative to standard techniques for environmental monitoring," Pervasive and Mobile Computing, 2012.

[24] A. Hevner and S. Chatterjee, "Design Science Research in Information Systems," in Design Research in Information Systems. vol. 22, ed: Springer US, 2010, pp. 9-22.

[25] A. R. Hevner, S. T. March, J. Park, and S. Ram, "Design Science in Information Systems Research," MIS Quarterly, vol. 28, pp. 75-105, 2004.

[26] K. Peffers, T. Tuunanen, M. A. Rothenberger, and S. Chatterjee, "A Design Science Research Methodology for Information Systems Research," Journal of Management Information Systems, vol. 24, pp. 45-77, 2007/12/01 2007.

[27] S. Gregor and A. R. Hevner, "Positioning and Presenting Design Science Research for Maximum Impact," vol. 37, ed: MIS Quarterly, 2013, pp. 337355.

[28] W. Kuechler and V. Vaishnavi, "The emergence of design research in information systems in North America," Journal of Design Research, vol. 7, pp. 1-16, 2008.

[29] S. Dibb, Marketing: Concepts and Strategies: Houghton Mifflin College Division, 2006.

[30] J. G. Lynch, "Memory and decision making," Handbook of consumer behavior, pp. 1-49, 1991.

[31] B. Schwartz, The paradox of choice: HarperCollins, 2009.

[32] S. M. Shugan, "The cost of thinking," Journal of consumer Research, pp. 99$111,1980$.

[33] K. B. Murray and G. Häubl, "Interactive consumer decision aids," in Handbook of marketing decision models, ed: Springer, 2008, pp. 55-77. 
[34] H. Assael, Consumer Behavior and Marketing Action. Boston, MA: PWSKENT, 1992.

[35] P. Kotler, Marketing management : analysis, planning, implementation, and control. London Prentice-Hall International, 1991.

[36] M. R. Solomon, Consumer Behaviour: A European Perspective: FT Prentice Hall, 2009.

[37] S. Changchien, C.-F. Lee, and Y.-J. Hsu, "On-line personalized sales promotion in electronic commerce," Expert Systems with Applications, vol. 27, pp. 35-52, 2004.

[38] P. Resnick and H. R. Varian, "Recommender systems," Communications of the ACM, vol. 40, pp. 56-58, 1997.

[39] G. Adomavicius, N. Manouselis, and Y. Kwon, "Multi-Criteria Recommender Systems," in Recommender Systems Handbook, F. Ricci, L. Rokach, B. Shapira, and P. B. Kantor, Eds., ed: Springer US, 2011, pp. 769-803.

[40] R. Burke, "Hybrid recommender systems: Survey and experiments," User modeling and user-adapted interaction, vol. 12, pp. 331-370, 2002.

[41] G. Adomavicius and $\mathrm{Y}$. Kwon, "New recommendation techniques for multicriteria rating systems," Intelligent Systems, IEEE, vol. 22, pp. 48-55, 2007.

[42] H. A. Simon, "A behavioral model of rational choice," The quarterly journal of economics, pp. 99-118, 1955.

[43] A. Tversky and D. Kahneman, "Judgment under uncertainty: Heuristics and biases," science, vol. 185, pp. 1124-1131, 1974.

[44] A. Campbell, S. Eisenman, N. Lane, E. Miluzzo, and R. Peterson, "Peoplecentric urban sensing," in WICON '06: Proceedings of the 2nd annual international workshop on Wireless internet, Boston, Massachusetts, 2006, p. 18.

[45] E. Paulos, R. Honicky, and E. Goodman, "Sensing Atmosphere," in ACM Conference on Embedded Networked Sensor Systems (SenSys 2007), Sydney, Australia, 2007. 
[46] O. Riva and C. Borcea, "The Urbanet revolution: Sensor power to the people!," IEEE Pervasive Computing, pp. 41-49, 2007.

[47] E. Kanjo, J. Bacon, D. Roberts, and P. Landshoff, "MobSens: Making Smart Phones Smarter," IEEE Pervasive Computing, pp. 50-57, 2009.

[48] C. T. Chou, N. Bulusu, and S. Kanhere, "Sensing data market," Proceedings of Poster Papers, p. 13, 2007.

[49] J. S. Lee and B. Hoh, "Sell Your Experiences: A Market Mechanism based Incentive for Participatory Sensing," in Eighth IEEE International Conference on Pervasive Computing and Communications (IEEE PerCom 2010), Mannheim, Germany, 2010.

[50] Y. Dong, S. Kanhere, C. Chou, and N. Bulusu, "Automatic Collection of Fuel Prices from a Network of Mobile Cameras," ed, 2008, pp. 140-156.

[51] S. Reddy, K. Shilton, G. Denisov, C. Cenizal, D. Estrin, and M. Srivastava, "Biketastic: sensing and mapping for better biking," in CHI '10: Proceedings of the 28th international conference on Human factors in computing systems, Atlanta, Georgia, USA, 2010, pp. 1817-1820.

[52] S. Reddy, A. Parker, J. Hyman, J. Burke, D. Estrin, and M. Hansen, "Image browsing, processing, and clustering for participatory sensing: lessons from a DietSense prototype," in EmNets '07: Proceedings of the 4th workshop on Embedded networked sensors, Cork, Ireland, 2007, pp. 13-17.

[53] E. Miluzzo, N. Lane, K. Fodor, R. Peterson, H. Lu, M. Musolesi, et al., "Sensing meets mobile social networks: the design, implementation and evaluation of the CenceMe application," in SenSys '08: Proceedings of the 6th ACM conference on Embedded network sensor systems, Raleigh, NC, USA, 2008, pp. 337-350.

[54] J. Eriksson, L. Girod, B. Hull, R. Newton, S. Madden, and H. Balakrishnan, "The pothole patrol: using a mobile sensor network for road surface monitoring," in MobiSys '08: Proceeding of the 6th international conference on Mobile systems, applications, and services, Breckenridge, CO, USA, 2008, pp. 29-39.

[55] S. B. Eisenman, E. Miluzzo, N. D. Lane, R. A. Peterson, G. S. Ahn, and A. T. Campbell, "BikeNet: A mobile sensing system for cyclist experience 
mapping," ACM Transactions on Sensor Networks (TOSN), vol. 6, pp. 1-39, 2009.

[56] M. Musolesi, E. Miluzzo, N. D. Lane, S. B. Eisenman, T. Choudhury, and A. T. Campbell, "The Second Life of a sensor: Integrating real-world experience in virtual worlds using mobile phones," Technology, vol. 100, p. 3, 2008.

[57] S. H. Wuyts, M. G. Dekimpe, E. Gijsbrechts, and F. R. Pieters, The connected customer: The changing nature of consumer and business markets: Routledge, 2010.

[58] F. Li, "Making Sense of the Connected Consumers: The Business Perspective," in Consumers and Internet Studies: a Workshop, Barcelona, Spain, 2011.

[59] JiWire. (2013, 20 January). Mobile Audience Insights Report, Q2 2013. Available: content/uploads/2013/09/JiWire_Insights_Q2_2013.pdf http://jiwire.com/wp-

[60] JiWire. (2013, 20 January). Mobile Audience Insights Report, Q3 2013. Available: content/uploads/2013/11/JiWire_Insights_Q3_2013.pdf

[61] N. Bulusu, C. T. Chou, S. Kanhere, Y. Dong, S. Sehgal, D. Sullivan, et al., "Participatory sensing in commerce: Using mobile camera phones to track market price dispersion," in Proceedings of the International Workshop on Urban, Community, and Social Applications of Networked Sensing Systems (UrbanSense 2008), 2008, pp. 6-10.

[62] S. Sehgal, S. S. Kanhere, and C. T. Chou, "\{MobiShop: Using Mobile Phones for Sharing Consumer Pricing Information\}," in Demo Session of the Intl. Conference on Distributed Computing in Sensor Systems, Santorini, Greece, 2008.

[63] R. Barnes, J. Hudgins, and A. Muse, "ShopSavvy," Android Market, 2009.

[64] F. von Reischach and F. Michahelles, "Apriori: A ubiquitous product rating system," in Workshop on Pervasive Mobile Interaction Devices (PERMID) at Pervasive 2008, 2008. 
[65] A. K. Dey and G. D. Abowd, "CybreMinder: A context-aware system for supporting reminders," in Handheld and Ubiquitous Computing, 2000, pp. 172-186.

[66] P. Nurmi, A. Salovaara, A. Forsblom, F. Bohnert, and P. Floréen, "PromotionRank: Ranking and Recommending Grocery Product Promotions Using Personal Shopping Lists," ACM Transactions on Interactive Intelligent Systems (TiiS), vol. 4, p. 1, 2014.

[67] S. Bhattacharya, P. Floreen, A. Forsblom, S. Hemminki, P. Myllymaki, P. Nurmi, et al., "Ma\$\$iv€ - An Intelligent Mobile Grocery Assistant," in Intelligent Environments (IE), 2012 8th International Conference on, 2012, pp. 165-172.

[68] M. Mun, S. Reddy, K. Shilton, N. Yau, J. Burke, D. Estrin, et al., "PEIR, the personal environmental impact report, as a platform for participatory sensing systems research," in MobiSys '09: Proceedings of the 7th international conference on Mobile systems, applications, and services, Krakow, Poland, 2009, pp. 55-68.

[69] N. Maisonneuve, M. Stevens, M. E. Niessen, and L. Steels, "Noisetube: Measuring and mapping noise pollution with mobile phones," Information Technologies in Environmental Engineering, pp. 215-228, 2009.

[70] X. Bao and R. Choudhury, "VUPoints: collaborative sensing and video recording through mobile phones," in MobiHeld '09: Proceedings of the 1st ACM workshop on Networking, systems, and applications for mobile handhelds, Barcelona, Spain, 2009, pp. 7-12.

[71] H. Lu, W. Pan, N. Lane, T. Choudhury, and A. Campbell, "SoundSense: scalable sound sensing for people-centric applications on mobile phones," in Mobisys '09: Proceedings of the 7th international conference on Mobile systems, applications, and services, Wroclaw, Poland, 2009, pp. 165-178.

[72] E. Kanjo, "NoiseSPY: A Real-Time Mobile Phone Platform for Urban Noise Monitoring and Mapping," Mob. Netw. Appl., vol. 15, pp. 562-574, 2010.

[73] R. Rana, C. T. Chou, S. Kanhere, N. Bulusu, and W. Hu, "Ear-Phone: An End-to-End Participatory Urban Noise Mapping System," 2009.

[74] P. Dutta, P. M. Aoki, N. Kumar, A. Mainwaring, C. Myers, W. Willett, et al., "Common Sense: participatory urban sensing using a network of handheld 
air quality monitors," presented at the Proceedings of the 7th ACM Conference on Embedded Networked Sensor Systems, Berkeley, California, 2009.

[75] P. Mohan, V. Padmanabhan, and R. Ramjee, "Nericell: rich monitoring of road and traffic conditions using mobile smartphones," in SenSys '08: Proceedings of the 6th ACM conference on Embedded network sensor systems, Raleigh, NC, USA, 2008, pp. 323-336.

[76] A. Thiagarajan, L. Ravindranath, K. LaCurts, S. Madden, H. Balakrishnan, S. Toledo, et al., "VTrack: accurate, energy-aware road traffic delay estimation using mobile phones," presented at the Proceedings of the 7th ACM Conference on Embedded Networked Sensor Systems, Berkeley, California, 2009.

[77] R. K. Ganti, N. Pham, H. Ahmadi, S. Nangia, and T. F. Abdelzaher, "GreenGPS: a participatory sensing fuel-efficient maps application," presented at the Proceedings of the 8th international conference on Mobile systems, applications, and services, San Francisco, California, USA, 2010.

[78] S. Mathur, T. Jin, N. Kasturirangan, J. Chandrasekaran, W. Xue, M. Gruteser, et al., "ParkNet: drive-by sensing of road-side parking statistics," presented at the Proceedings of the 8th international conference on Mobile systems, applications, and services, San Francisco, California, USA, 2010.

[79] P. Nam, R. K. Ganti, N. Saurabh, T. Pongthawornkamol, A. Shameem, T. F. Abdelzaher, et al., "Fueoogle: A Participatory Sensing Fuel-Efficient Maps Application," Status: unpublished, 2009.

[80] E. Miluzzo, N. Lane, S. Eisenman, and A. Campbell, "CenceMe - Injecting Sensing Presence into Social Networking Applications," ed, 2007, pp. 1-28.

[81] L. R. Inc. (05.25). Second Life. Available: http://secondlife.com/

[82] X. Bao and R. R. Choudhury, "MoVi: mobile phone based video highlights via collaborative sensing," presented at the Proceedings of the 8th international conference on Mobile systems, applications, and services, San Francisco, California, USA, 2010.

[83] A. Beach, M. Gartrell, X. Xing, R. Han, Q. Lv, S. Mishra, et al., "Fusing mobile, sensor, and social data to fully enable context-aware computing," in 
HotMobile '10: Proceedings of the Eleventh Workshop on Mobile Computing Systems \& Applications, Annapolis, MD, USA, 2010, pp. 60-65.

[84] N. Banerjee, S. Agarwal, P. Bahl, R. Chandra, A. Wolman, and M. Corner, "Virtual Compass: Relative Positioning to Sense Mobile Social Interactions," in Pervasive Computing. vol. 6030, P. Floréen, A. Krüger, and M. Spasojevic, Eds., ed: Springer Berlin / Heidelberg, 2010, pp. 1-21.

[85] S. B. Eisenman, E. Miluzzo, N. D. Lane, R. A. Peterson, G. S. Ahn, and A. T. Campbell, "The BikeNet mobile sensing system for cyclist experience mapping," in SenSys '07: Proceedings of the 5th international conference on Embedded networked sensor systems, Sydney, Australia, 2007, pp. 87-101.

[86] K. Sha, G. Zhan, W. Shi, M. Lumley, C. Wiholm, and B. Arnetz, "SPA: a smart phone assisted chronic illness self-management system with participatory sensing," in HealthNet '08: Proceedings of the 2nd International Workshop on Systems and Networking Support for Health Care and Assisted Living Environments, Breckenridge, Colorado, 2008, pp. 1-3.

[87] S. Consolvo, D. W. McDonald, T. Toscos, M. Y. Chen, J. Froehlich, B. Harrison, et al., "Activity sensing in the wild: a field trial of ubifit garden," presented at the Proceeding of the twenty-sixth annual SIGCHI conference on Human factors in computing systems, Florence, Italy, 2008.

[88] G. S. Thakur, M. Sharma, and A. Helmy, "SHIELD: Social sensing and Help In Emergency using mobiLe Devices," Arxiv preprint arXiv:1004.4356, 2010.

[89] A. Kapadia, N. Triandopoulos, C. Cornelius, D. Peebles, and D. Kotz, "AnonySense: Opportunistic and Privacy-Preserving Context Collection," Pervasive Computing, vol. 5013, pp. 280-297, 2008.

[90] M. Shin, C. Cornelius, D. Peebles, A. Kapadia, D. Kotz, and N. Triandopoulos, "AnonySense: A system for anonymous opportunistic sensing," Pervasive and Mobile Computing, 2010.

[91] A. Joki, J. A. Burke, and D. Estrin, "Campaignr: A framework for participatory data collection on mobile phones," 2007.

[92] D. Trossen and D. Pavel, "NORS: An Open Source Platform to Facilitate Participatory Sensing with Mobile Phones," presented at the Proceedings of the 2007 Fourth Annual International Conference on Mobile and Ubiquitous Systems: Networkingl\&Services (MobiQuitous), 2007. 
[93] T. Das, P. Mohan, V. N. Padmanabhan, R. Ramjee, and A. Sharma, "PRISM: Platform for Remote Sensing using Smartphones," 2010.

[94] M. Zhang, A. Joshi, R. Kadmawala, K. Dantu, S. Poduri, and G. S. Sukhatme, "OCRdroid: A Framework to Digitize Text Using Mobile Phones," in Mobile Computing, Applications, and Services, ed: Springer, 2010, pp. 273-292.

[95] R. Smith, "An overview of the Tesseract OCR engine," in Document Analysis and Recognition, 2007. ICDAR 2007. Ninth International Conference on, 2007, pp. 629-633.

[96] S. Tokunaga, S. Matsumoto, and M. Nakamura, "Receiptlog: A consumeroriented lifelog service for storing and reviewing daily receipts," iiWAS2011, vol. 111, pp. 23-28, 2011.

[97] S. C. Park, M. K. Park, and M. G. Kang, "Super-resolution image reconstruction: a technical overview," Signal Processing Magazine, IEEE, vol. 20 , pp. 21-36, 2003

[98] C. A. Segall, R. Molina, and A. K. Katsaggelos, "High-resolution images from low-resolution compressed video," Signal Processing Magazine, IEEE, vol. 20 , pp. 37-48, 2003.

[99] P. Vandewalle, S. Sü, and M. Vetterli, "A frequency domain approach to registration of aliased images with application to super-resolution," EURASIP Journal on Advances in Signal Processing, vol. 2006, 2006.

[100] S. Farsiu, M. D. Robinson, M. Elad, and P. Milanfar, "Fast and robust multiframe super resolution," Image processing, IEEE Transactions on, vol. 13, pp. 1327-1344, 2004.

[101] N. Nguyen, P. Milanfar, and G. Golub, "A computationally efficient superresolution image reconstruction algorithm," Image Processing, IEEE Transactions on, vol. 10, pp. 573-583, 2001.

[102] L. I. Rudin, S. Osher, and E. Fatemi, "Nonlinear total variation based noise removal algorithms," Physica D: Nonlinear Phenomena, vol. 60, pp. 259-268, 1992.

[103] A. Zomet, A. Rav-Acha, and S. Peleg, "Robust super-resolution," in Computer Vision and Pattern Recognition, 2001. CVPR 2001. Proceedings 130 
of the 2001 IEEE Computer Society Conference on, 2001, pp. I-645-I-650 vol. 1.

[104] S. T. March and V. C. Storey, "Design science in the information systems discipline: an introduction to the special issue on design science research," MIS Q., vol. 32, pp. 725-730, 2008.

[105] R. M. O'keefe and T. McEachern, "Web-based customer decision support systems," Communications of the ACM, vol. 41, pp. 71-78, 1998.

[106] E. Newcomb, T. Pashley, and J. Stasko, "Mobile computing in the retail arena," in Proceedings of the SIGCHI conference on Human factors in computing systems, 2003, pp. 337-344.

[107] C. Pescher, P. Reichhart, and M. Spann, "Consumer Decision-making Processes in Mobile Viral Marketing Campaigns," Journal of Interactive Marketing, vol. 28, pp. 43-54, 2// 2014.

[108] R. Ström, M. Vendel, and J. Bredican, "Mobile marketing: A literature review on its value for consumers and retailers," Journal of Retailing and Consumer Services, vol. 21, pp. 1001-1012, 11// 2014.

[109] K. Z. K. Zhang, S. J. Zhao, C. M. K. Cheung, and M. K. O. Lee, "Examining the influence of online reviews on consumers' decision-making: A heuristicsystematic model," Decision Support Systems, vol. 67, pp. 78-89, 2014.

[110] W. Duan, B. Gu, and A. B. Whinston, "The dynamics of online word-of-mouth and product sales-An empirical investigation of the movie industry," Journal of Retailing, vol. 84, pp. 233-242, 2008.

[111] P. Katz, T. Lunze, M. Feldmann, D. Röhrborn, and A. Schill, "System Architecture for Handling the Information Overload in Enterprise Information Aggregation Systems," in Business Information Systems. vol. 87, W. Abramowicz, Ed., ed: Springer Berlin Heidelberg, 2011, pp. 148-159.

[112] S. Shekar, P. Nair, and A. Helal, "iGrocer: a ubiquitous and pervasive smart grocery shopping system," in Proceedings of the 2003 ACM symposium on Applied computing, Melbourne, Florida, 2003, pp. 645-652.

[113] J. Sun, "Information requirement elicitation in mobile commerce," Communications of the ACM, vol. 46, pp. 45-47, 2003. 
[114] S. J. Westerman, G. C. Tuck, S. A. Booth, and K. Khakzar, "Consumer decision support systems: Internet versus in-store application," Computers in Human Behavior, vol. 23, pp. 2928-2944, 11// 2007.

[115] N. H. Lurie and N. Wen, "Simple Decision Aids and Consumer Decision Making," Journal of Retailing, vol. 90, pp. 511-523, 12// 2014.

[116] G. Häubl and V. Trifts, "Consumer decision making in online shopping environments: The effects of interactive decision aids," Marketing science, vol. 19, pp. 4-21, 2000.

[117] R. E. Pereira, "Influence of Query-Based Decision Aids on Consumer Decision Making in Electronic Commerce," Information Resources Management Journal (IRMJ), vol. 14, pp. 31-48, 2001.

[118] S. Senecal and J. Nantel, "The influence of online product recommendations on consumers' online choices," Journal of Retailing, vol. 80, pp. 159-169, // 2004.

[119] S. Keegan, G. M. O'Hare, and M. J. O'Grady, "Easishop: Ambient intelligence assists everyday shopping," Information Sciences, vol. 178, pp. 588-611, 2008.

[120] W. G. Mangold and D. J. Faulds, "Social media: The new hybrid element of the promotion mix," Business Horizons, vol. 52, pp. 357-365, 7// 2009.

[121] A. Dey and G. Abowd, "CybreMinder: A Context-Aware System for Supporting Reminders," in Handheld and Ubiquitous Computing. vol. 1927, P. Thomas and H.-W. Gellersen, Eds., ed: Springer Berlin Heidelberg, 2000, pp. 172-186.

[122] R. D. Lawrence, G. S. Almasi, V. Kotlyar, M. S. Viveros, and S. S. Duri, "Personalization of Supermarket Product Recommendations," Data Mining and Knowledge Discovery, vol. 5, pp. 11-32, 2001/01/01 2001.

[123] P. Butler and J. Peppard, "Consumer purchasing on the Internet: Processes and prospects," European management journal, vol. 16, pp. 600-610, 1998.

[124] J. Jacoby, D. E. Speller, and C. A. Kohn, "Brand choice behavior as a function of information load," Journal of Marketing Research, pp. 63-69, 1974. 
[125] B. K. Lee and W. N. Lee, "The effect of information overload on consumer choice quality in an online environment," Psychology \& Marketing, vol. 21, pp. 159-183, 2004.

[126] C. A. O'Reilly, "Individuals and information overload in organizations: is more necessarily better?," Academy of management journal, vol. 23, pp. 684-696, 1980.

[127] V. Y. Yoon, R. E. Hostler, Z. Guo, and T. Guimaraes, "Assessing the moderating effect of consumer product knowledge and online shopping experience on using recommendation agents for customer loyalty," Decision Support Systems, vol. 55, pp. 883-893, 11// 2013.

[128] M. Aljukhadar, S. Senecal, and C.-E. Daoust, "Using Recommendation Agents to Cope with Information Overload," International Journal of Electronic Commerce, vol. 17, pp. 41-70, 01/01/2012.

[129] Y.-C. Chen, R.-A. Shang, and C.-Y. Kao, "The effects of information overload on consumers' subjective state towards buying decision in the internet shopping environment," Electronic Commerce Research and Applications, vol. 8, pp. 48-58, 1// 2009.

[130] J. R. Hauser and B. Wernerfelt, "An evaluation cost model of consideration sets," Journal of consumer research, pp. 393-408, 1990.

[131] M. Hepp, "GoodRelations: An Ontology for Describing Products and Services Offers on the Web," in Knowledge Engineering: Practice and Patterns. vol. 5268, A. Gangemi and J. Euzenat, Eds., ed: Springer Berlin Heidelberg, 2008, pp. 329-346.

[132] M. P. Friedman, "Consumer confusion in the selection of supermarket products," Journal of Applied Psychology, vol. 50, p. 529, 1966.

[133] S. Dibb, L. Simkin, W. M. Pride, and O. C. Ferrell, Marketing: Concepts and Strategies. 5th Edition. Abingdon, UK: Houghton Mifflin, 2005.

[134] W.-T. Wang and W.-H. Chang, "A study of virtual product consumption from the expectancy disconfirmation and symbolic consumption perspectives," Information Systems Frontiers, vol. 16, pp. 887-908, 2014/11/01 2014. 
[135] L. G. Block and V. G. Morwitz, "Shopping lists as an external memory aid for grocery shopping: Influences on list writing and list fulfillment," Journal of Consumer Psychology, vol. 8, pp. 343-375, 1999.

[136] A. Thomas and R. Garland, "Grocery shopping: list and non-list usage," Marketing Intelligence \& Planning, vol. 22, pp. 623-635, 2004.

[137] S. Ozarslan and P. E. Eren, "Text recognition and correction for automated data collection by mobile devices," in IS\&T/SPIE Electronic Imaging, 2014, pp. 902706-902706-6.

[138] R. J. Kauffman, H. Lai, and C.-T. Ho, "Incentive mechanisms, fairness and participation in online group-buying auctions," Electronic Commerce Research and Applications, vol. 9, pp. 249-262, 5// 2010.

[139] D. Yi Fei, S. Kanhere, C. Chun Tung, and L. Ren Ping, "Automatic image capturing and processing for PetrolWatch," in Networks (ICON), 2011 17th IEEE International Conference on, 2011, pp. 236-240.

[140] S. Ozarslan and P. E. Eren, "A Mobile Participatory Framework for the Consumer Decision Process," in Next Generation Mobile Apps, Services and Technologies (NGMAST), 2013 Seventh International Conference on, 2013, pp. 93-98.

[141] P. Ye and D. Doermann, "Document Image Quality Assessment: A Brief Survey," in Document Analysis and Recognition (ICDAR), 2013 12th International Conference on, 2013, pp. 723-727.

[142] J. Liang, D. Doermann, and H. Li, "Camera-based analysis of text and documents: a survey," International Journal of Document Analysis and Recognition (IJDAR), vol. 7, pp. 84-104, 2005.

[143] F. Boschetti, M. Romanello, A. Babeu, D. Bamman, and G. Crane, "Improving OCR accuracy for classical critical editions," in Research and Advanced Technology for Digital Libraries, ed: Springer, 2009, pp. 156-167.

[144] V. I. Levenshtein, "Binary codes capable of correcting deletions, insertions and reversals," in Soviet physics doklady, 1966, p. 707.

[145] R. Xu and D. Wunsch, "Survey of clustering algorithms," Neural Networks, IEEE Transactions on, vol. 16, pp. 645-678, 2005. 
[146] T. L. Saaty, "Decision making - the analytic hierarchy and network processes (AHP/ANP)," Journal of systems science and systems engineering, vol. 13, pp. 1-35, 2004.

[147] P. C. Fishburn, "Additive Utilities with Incomplete Product Sets: Application to Priorities and Assignments," Operations Research, vol. 15, pp. 537-542, 1967.

[148] J. Figueira, V. Mousseau, and B. Roy, "ELECTRE methods," in Multiple criteria decision analysis: State of the art surveys, ed: Springer, 2005, pp. 133-153.

[149] J. Fülöp, "Introduction to decision making methods," in BDEl-3 Workshop, Washington, 2005.

[150] J.-P. Brans and P. Vincke, "Note-A Preference Ranking Organisation Method (The PROMETHEE Method for Multiple Criteria Decision-Making)," Management science, vol. 31, pp. 647-656, 1985.

[151] B. Sebastien, B. Raymond, V. Thomas, and M. Patrick. (22 May 2013). Decision Deck XMCDA Web Services. Available: http://www.decisiondeck.org/ws/

[152] W. ISO, "9241-11. Ergonomic requirements for office work with visual display terminals (VDTs)," The international organization for standardization, 1998.

[153] J. Brooke, "SUS-A quick and dirty usability scale," in Usability evaluation in industry, P. W. Jordan, B. Thomas, B. A. Weerdmeester, and I. L. McClelland, Eds., ed London: Taylor \& Francis, 1996, pp. 189-194.

[154] A. Bangor, P. T. Kortum, and J. T. Miller, "An empirical evaluation of the system usability scale," Intl. Journal of Human-Computer Interaction, vol. 24, pp. 574-594, 2008.

[155] J. Nielsen and T. K. Landauer, "A mathematical model of the finding of usability problems," in Proceedings of the INTERACT'93 and CHI'93 conference on Human factors in computing systems, 1993, pp. 206-213.

[156] T. S. Tullis and J. N. Stetson, "A comparison of questionnaires for assessing website usability," in Usability Professional Association Conference, Minneapolis, MN, 2004, pp. 1-12. 
[157] L. Faulkner, "Beyond the five-user assumption: Benefits of increased sample sizes in usability testing," Behavior Research Methods, Instruments, \& Computers, vol. 35, pp. 379-383, 2003.

[158] A. Bangor, P. Kortum, and J. Miller, "Determining what individual SUS scores mean: Adding an adjective rating scale," Journal of usability studies, vol. 4, pp. 114-123, 2009.

[159] J. Sauro, A practical guide to the system usability scale: Background, benchmarks \& best practices: Measuring Usability LLC, 2011.

[160] Ookla. (20 January 2014). Speedtest.net by Ookla - The Global Broadband Speed Test. Available: http://www.speedtest.net/

[161] B. Roy, "The outranking approach and the foundations of ELECTRE methods," Theory and decision, vol. 31, pp. 49-73, 1991. 


\title{
CURRICULUM VITEA
}

\author{
SÜLEYMAN ÖZARSLAN \\ Information Systems Department \\ Middle East Technical University \\ Informatics Institute, Universiteler Mahallesi, Dumlupınar Bulvarı, No:1, 06800, \\ Ankara,Turkey \\ TEL: +90 31221077002 FAX: +90 3122103745 \\ EMAIL: ozarslan@metu.edu.tr
}

\author{
AREAS OF INTEREST \\ - Participatory Sensing \\ - Sensor Networks \\ - Information and network security \\ - Computer and communications networks \\ EDUCATION \\ Middle East Technical University, Ankara, Turkey \\ Doctor of Philosophy in Information Systems \\ $2008-2015$ \\ Thesis Title: MobileCDP: A Mobile Framework for the Consumer Decision \\ Process \\ Advisor: Assist. Prof. Dr. P. Erhan Eren \\ Middle East Technical University, Ankara, Turkey \\ Master of Science in Information Systems \\ 2005-2008
}


Thesis Title: Simulation of a Mobile Agent Middleware for Wireless Sensor Networks

Advisor: Prof. Dr. Yasemin Yardımcı

Co-advisor: Assoc. Prof. Dr. Y. Murat Erten

Ege University, Izmir, Turkey

Bachelor of Science in Mathematics

$1998-2003$

\section{TEACHING ASSISTANTSHIPS}

- Mobile and Pervasive Computing, Assistant

- Sensor Networks, Assistant.

- Information Security Management, Assistant.

- Security Engineering, Assistant.

- Data Base Concepts and Applications, Assistant.

- Object Oriented Programming and Data Structures, Assistant.

- Artificial Intelligence, Assistant.

- IT Governance, Assistant.

- Regulatory and Legal Aspects of Information Systems, Assistant

- Introduction to Information Technology and Applications, Instructor

\section{PAPERS}

Özarslan, S., Eren P.E., "Multi-frame Knowledge Based Text Enhancement for Mobile Phone Captured Videos," Proc. SPIE 9030, Mobile Devices and Multimedia: Enabling Technologies, Algorithms, and Applications 2014, (2014).

Özarslan, S., Eren P.E., "Text recognition and correction for automated data collection by mobile devices," Proc. SPIE 9027, Imaging and Multimedia Analytics in a Web and Mobile World 2014, (2014).

Özarslan, S., Eren P.E., "Comparison of Approaches for Mobile Document Image Analysis Using server supported smartphones," Proc. SPIE 9023, Digital Photography X 2014, (2014). 
Özarslan, S., Eren, P. E., "A Mobile Participatory Framework for the Consumer Decision Process," Proc. Seventh International Conference on Next Generation Mobile Apps, Services and Technologies (NGMAST), 93-98 (2013).

Özarslan, S., Eren, P. E. "Katılımcı Algılama Uygulamalarında Otomatik Veri Toplama İçin Karakter Tanıma ve Düzeltme", Signal Processing and Communications Applications Conference (SIU), (2013).

Özarslan, S., Erten, M. (2008). Simulation of Agilla middleware on TOSSIM. International Conference on Simulation Tools and Techniques for Communications, Networks and Systems \& Workshops.

Babayev Dj. A., Nuriyev U.G., Özarslan S, Sözeri V. Kapalı sayılar üzerine, XIX. Ulusal Matematik Sempozyumu, 22-25 Ağustos, Kütahya, 2006.

Babayev D.A., Nuriyev U.G., Özarslan S., "Locked numbers and their application in information Technologies", Proceeding of the International scientific conference "Information Technologies and Telecommunications in Education and Science" (IT \& T ES'2005), p.190-194, May 15-22, 2005.

Nuriyev U.G., Özarslan S., Akleylek S., "Securty of Online Learning", Proceeding o the flnternational scientific conference "Information Technologies and Telecommunications in Education and Science" (IT \& T ES'2005), p. 194-196, May 15-22, 2005.

Özarslan, S., Nuriyev, U.G.,"Kuantum Kriptografisi ile \%100 Bilgi güvenliği”, III Bilgi Teknolojileri Kongresi (BILLGiTEK'2004), Bildiriler kitabı, 43 - 46. Denizli, Pamukkale Üniversitesi, 2004.

Özarslan, S., Nuriyev, U.G., "íkiz Asal Sayıların Kullanıldığı bir Şifreleme Algoritması", Savunma Teknolojileri Kongresi - SAVTEK 2002, Bildiriler Kitabi, Cilt 1, 417 - 426, Ankara, ODTÜ, 2002.

\section{JOURNAL PAPERS SUBMITTED FOR REVIEW}

- MobileCDP: A Mobile Framework for the Consumer Decision Process

- Multi-frame Knowledge Based Text Extraction and Enhancement for Mobile Phone Captured Videos

\section{ACADEMIC REFEREEINGS}

- - Member of the Programme Committee of NGMAST (2014 - )

- $\quad$ - Reviewer of JEI - Journal of Electronic Imaging (2014 - ) 